\title{
Dynamic Control of Centrifugal Compressor Surge Using Tailored Structure
}

by

Daniel Lawrence Gysling

B.S. Aerospace Engineering

The Pennsylvania State University (1987)

Submitted to the Department of Aeronautics and Astronautics in Partial Fulfillment of the Requirements for the Degree of

Master of Science in Aeronautics and Astronautics

at the

Massachusetts Institute of Technology

August 1989

(C) Massachusetts Institue of Technology, 1989, All Rights Reserved

Signature of Author

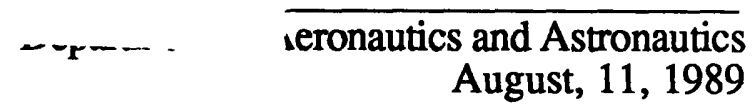

Certified by

Professor J. Dugundji

Professor of Aeronautics and Astronautics

Thesis Supervisor

Certified by

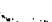

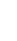




\title{
Dynamic Control of Centrifugal Compressor Surge Using Tailored Structure
}

by

\author{
Daniel Lawrence Gysling \\ Submitted to the Department of Aeronautics and Astronautics \\ on August 11, 1989 in partial fulfillment of the \\ requirements for the Degree of Master of Science in \\ Aeronauitics and Astronautics
}

\begin{abstract}
A new method for dynamic control of centrifugal compressor surge is developed analytically and demonstrated experimentally. The method suppresses surge by modifying the compression system dynamics using structural feedback. The volume of the plenum is made flexible to respond to small perturbations in plenum pressure, via a movable wall modelled as a mass-spring-damper. The wall motion provides additional system damping to extend the stable operating range of the compression system.

A lumped parameter model of the flexible wall compression system is developed and a set of non-dimensional structural control parameters which govern the interaction of the wall and system dynamics is presented. The basic compression system is modelled as a Helmholtz resonator and is coupled to the wall dynamics. The effectiveness of the wall is shown to be a function of the structural control parameters in addition to the basic compression system parameters. Unsteady energy transferred from flow perturbations to the wall and dissipated by the damper is shown to be the dominant stabilizing mechanism.

Steady state and transient data demonstrated that the lumped parameter model can accurately predict surge dynamics. As predicted by the model and demonstrated with experiment, a properly tuned flexible plenum wall significantly extended the stable flow range of a centrifugal compression system 25 to $30 \%$ over a large range of operating conditions. The flexible wall compression system was primarily designed based on linear theory. Control was achieved with relatively little power being dissipated through the flexible wall control system. Non-linear calculations showed that the amount of unsteadiness in the stabilized region is a function of non-linearities in the wall dynamics and these effects are quantified. Surge was supressed with no noticeable decrease in steady performance. The flexible plenum wall demonstrated the ability to suppress existing surge.
\end{abstract}

Thesis Supervisor: Dr. John Dugundji

Title: $\quad$ Professor of Aeronautics and Astronautics

Thesis Supervisor: Dr. Edward M. Greitzer

Title: $\quad$ Professor of Aeronautics and Astronautics 


\section{ACKNOWLEDGEMENTS}

The author wishes to thank all those who helped to smooth the road for the completion of this project, in particular:

Professor Dugundji and Professor Greitzer for their insight, guidance and encouragement throughout this project this project

Dr. Gerry Guenette for his help in sorting out the mechanical and electrical aspects of

Professor Epstein for his advice throughout this project, especially during the mechanical design stages

John Simon and Judy Pinsley for their help with running the rig and the data acquistion system

Lab

Jim Nash, Roy Andrew, and Viktor Dubrowski for all their advice and assistance in the

Bob Haimes for his help with the computing facilities in the Lab

and special thanks go to my family and friends for helping me through.

The project was conducted at the Gas Turbine Lab under a grant from the Air Force Office of Scientific Research, Dr. J. M. McMicheal and Capt. H. Helin, program managers. Financial support for the author was provided by the Air Force Research in Aero Propulsion Technology (AFRAPT) program, AFSOR-85-0288. 


\section{TABLE OF CONTENTS}

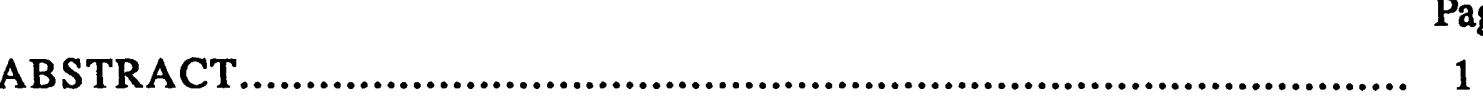

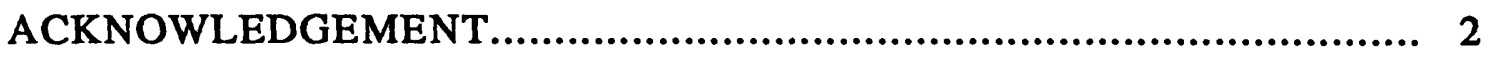

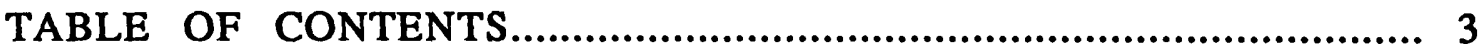

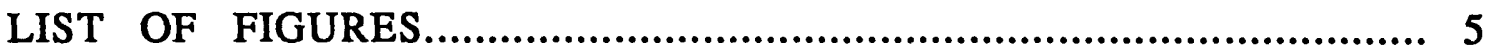

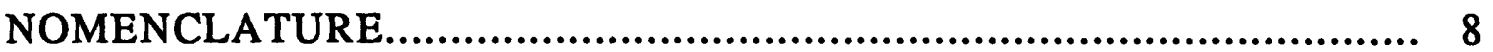

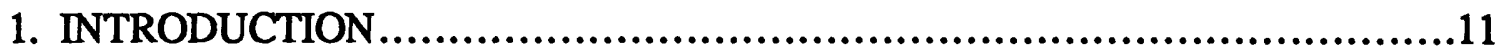

1.1 DESCRIPTION OF THE PROBLEM ...................................11

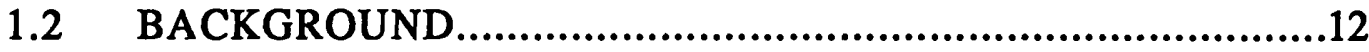

a. Compression System Model.......................................12

b. Previous Work on Surge Suppression .............................13

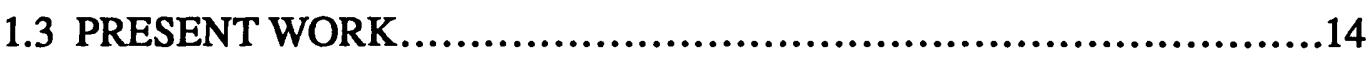

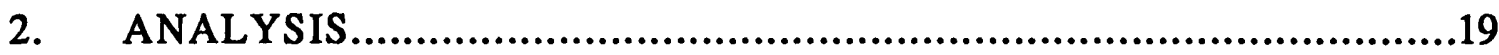

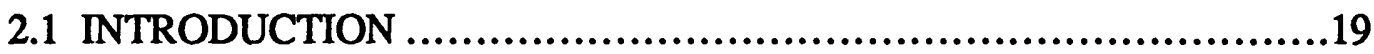

2.2 COMPRESSION SYSTEM MODELLING.................................19

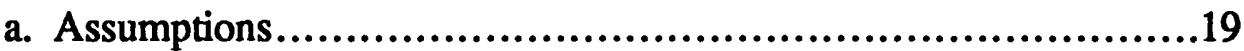

b. Equations for the System Dynamic Performance ....................20

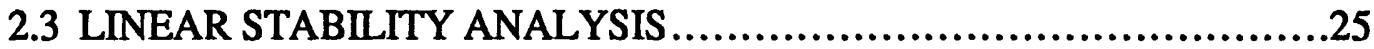

a. Fixed Wall Compression System .................................26

b. Flexible Plenum Wall System ...................................27

2.4 ENERGY BALANCE .................................................30

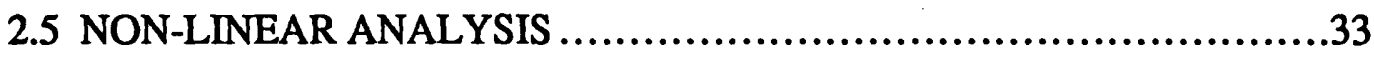

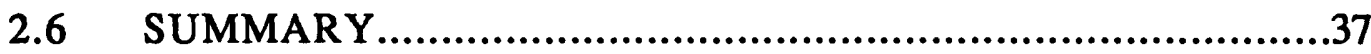

3. DESIGN OF THE EXPERIMENTAL FACILITY ...............................51

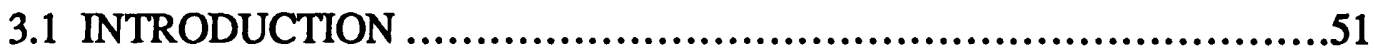

3.2 EXISTING FACILITIES ...............................................51

3.3 DESIGN OF FLEXIBLE PLENUM WALL .............................52

a. Aerodynamic Spring............................................52

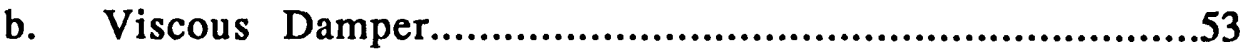

3.4 RIG INSTRUMENTATION ..........................................55

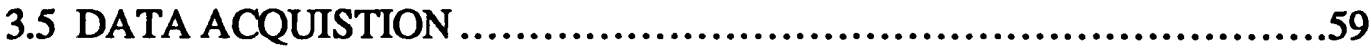

4. EXPERIMENTAL DATA AND ANALYSIS...................................74

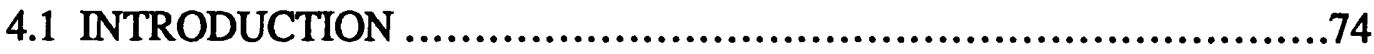

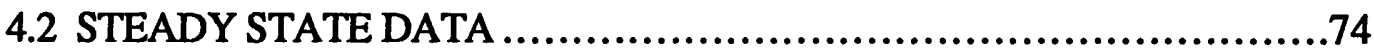

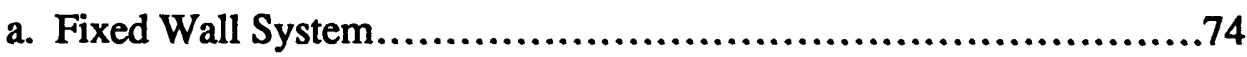


b. Flexible Wall System............................................77

c. Comparison with Theory....................................................79

4.3 TRANSIENT SYSTEM BEHAVIOR ................................82

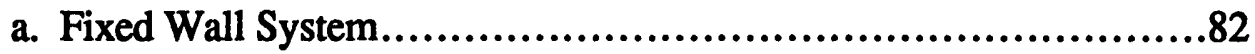

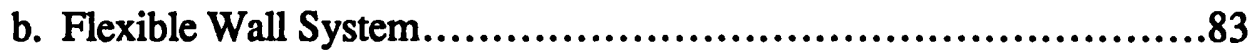

c. Comparison to Theory........................................................85

4.4 VALIDATION OF ASSUMPTIONS.................................86

a. Quasi-steady Compressor Slope ....................................86

b. Assumption of Constant Wheel Speed ................................88

4.5 COMPONENT PERFORMANCE........................................89

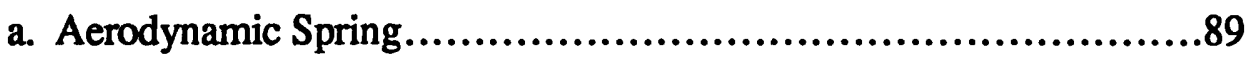

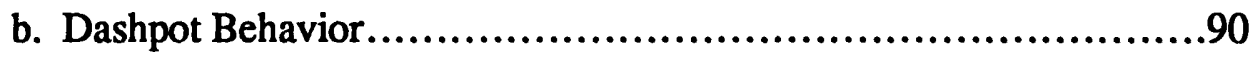

4.6 SUMMARY OF RESULTS .........................................91

5. CONCLUSIONS AND RECOMMENDATIONS.....................................119

5.1 CONCLUSIONS FROM EXPERIMENT................................. 119

5.2 CONCLUSIONS FROM ANALYSIS .................................120

5.3 RECOMMENDATIONS FOR FUTURE WORK .......................121

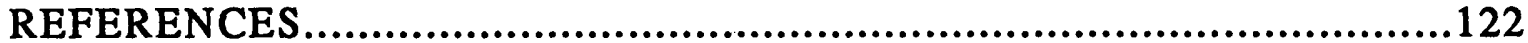

APPENDIX A. DERIVATION OF THE EQUATIONS OF MOTION FOR THE

FLEXIBLE PLENUM WALL SYSTEM.....................124

APPENDIX B. ALTERNATIVE SCHEMES FOR PASSIVE SURGE

SUPPRESSION 


\section{6: The Flexible Plenum Wall}

3.7: Inlet Hot Wire Calibration

3.8: Frequency to Voltage Converter Calibration

3.9: Main Plenum Pressure Transducer Calibration

3.10: Displacement Transducer Calibration

3.11: Damping Force Calibration

3.12: Auxiliary Plenum Pressure Calibration

3.13: Exit Mass Flow Orifice Pressure Transducer Calibration

3.14: Empirical Orifice Discharge Coefficient versus Reynolds Number

4.1: $\quad$ Fixed Wall Compressor Performance Map

4.2: Non-dimensional Fixed Wall Performance Map

4.3: Compressor Performance Map Fixed and Flexible Wall

4.4: Amplitude of Limit Cycle in Stablized Region ( $B=1.83$ )

4.5: Comparison of Close Coupled and Structurally Stabilized Speedline $(70 \mathrm{~K})$

4.6: Predicted and Experimental Surgelines Fixed Wall and Optimized Systems

4.7: Close Coupled Speedlines for Holset H1D (from Pinsley 1988)

4.8: Predicted versus Experimental Mass Flow Coefficients at Deep Surge (Fixed Wall and Optimized Systems)

4.9: Suppression of Fixed Wall Deep Surge with Flexible Wall

4.10: Amplitude of Plenum Pressure Fluctations Theory versus Experiment

4.11: Steady Operating Points for Transient Data

4.12: Fixed Wall Transient Behavior

4.13: Surge Cycles for Fixed Wall System (Points $C$ and E)

4.14: Frequency Spectrum of Fixed Wall Mild Surge Cycle

4.15A: Flexible Wall System Behavior

4.15B: Wall Motion for Points F - J 
4.16: Surge Cycles for Flexible Wall System (Points G and J)

4.17: Frequency Spectrum for Flexible Wall Surge Cycles Optimized Configuration

4.18A: Experimental and Calculated Mild Surge Cycles

4.18B: Calculated System Behavior Matching Amplitude of Pressure Limit Cycle to Experimental Data

4.19: Comparison of Calculated versus Experimental Wall Motion Matching Amplitude of Pressure Limit Cycle

4.20: Measured Instantaneous Compressor Slope versus Phi

4.21A: Wheel Speed and Plenum Pressure Fluctations During Fixed Wall Mild Surge

4.21B: Wheel Speed and Plenum Pressure FluctuationsDuring Fixed Wall Deep Surge

4.22: Aerodynamic Spring Force versus Displacement During Deep Surge

4.23: Aerodynamic Spring Constant Calculated in Frequency Domain

4.24: Wall Motion And Damper Force in Stabilized Region Medium Damping Configuration

4.25: Damping Constant as Function of Frequency and Frequency Spectrum of Wall Motion In Stabilized Region Medium Damping Configuration 


\section{NOMENCLATURE}

\section{Symbols}

\begin{tabular}{|c|c|c|}
\hline a & -- & speed of sound \\
\hline A & $\begin{array}{l}-- \\
--\end{array}$ & $\begin{array}{l}\text { inertia parameter } \\
\text { area }\end{array}$ \\
\hline B & -- & stability parameter \\
\hline c & -- & damping constant \\
\hline $\mathrm{C}$ & -- & flow velocity \\
\hline $\mathrm{f}$ & -- & Coulomb friction force \\
\hline $\mathrm{F}$ & -- & non-dimensional Coulomb friction force \\
\hline $\mathrm{i}$ & -- & imaginary number \\
\hline I & -- & rotational inertia of turbospool \\
\hline k & - & spring constant \\
\hline $\mathrm{L}$ & -- & effective length \\
\hline $\mathrm{m}$ & -- & mass of plenum wall \\
\hline$\dot{\mathrm{m}}$ & -- & mass flow \\
\hline M & -- & Mach number \\
\hline$P$ & -- & pressure \\
\hline q & -- & wall displacement \\
\hline Q & -- & wall frequency parameter \\
\hline $\mathrm{rms}$ & -- & root mean square \\
\hline $\mathrm{R}$ & -- & radius \\
\hline $\operatorname{Re}$ & -- & Reynolds number \\
\hline $\mathrm{U}$ & -- & impeller exit tip speed \\
\hline $\mathbf{v}$ & -- & non-dimensional wall motion \\
\hline V & -- & volume \\
\hline
\end{tabular}




\section{NOMENCLATURE}

Symbols

$\begin{array}{lll}\text { a } & -- & \text { speed of sound } \\ \text { A } & -- & \text { inertia parameter } \\ & -- & \text { area } \\ \text { B } & -- & \text { stability parameter } \\ \text { C } & -- & \text { damping constant } \\ \text { C } & -- & \text { flow velocity } \\ \text { f } & -- & \text { Coulomb friction force } \\ \text { F } & -- & \text { non-dimensional Coulomb friction force } \\ \text { i } & -- & \text { imaginary number } \\ \text { I } & -- & \text { rotational inertia of turbospool } \\ \text { k } & -- & \text { spring constant } \\ \text { L } & -- & \text { effective length } \\ \mathrm{m} & -- & \text { mass of plenum wall } \\ \dot{\mathrm{m}} & -- & \text { mass flow } \\ \mathrm{M} & -- & \text { Mach number } \\ \mathrm{P} & -- & \text { pressure } \\ \mathrm{q} & -- & \text { wall displacement } \\ \mathrm{Q} & -- & \text { wall frequency parameter } \\ \mathrm{rms} & -- & \text { root mean square } \\ \mathrm{R} & -- & \text { radius } \\ \mathrm{Re} & -- & \text { Reynolds number } \\ \mathrm{U} & -- & \text { impeller exit tip speed } \\ \mathrm{V} & -- & \text { non-dimensional wall motion } \\ \mathrm{V} & -- & \text { volume } \\ & & \\ & -\end{array}$




\begin{tabular}{|c|c|c|}
\hline W & $\begin{array}{l}-- \\
--\end{array}$ & $\begin{array}{l}\text { wall aeroelastic coupling parameter } \\
\text { work over a cycle }\end{array}$ \\
\hline $\mathrm{x}$ & -- & wall velocity \\
\hline$\alpha$ & .- & orifice discharge coefficient \\
\hline$\beta$ & -- & empirical orifice constant \\
\hline$\gamma$ & -- & ratio of specific heats \\
\hline$\delta^{*}$ & -- & boundary layer displacement thickness \\
\hline$\varepsilon$ & -- & compressibility factor \\
\hline$\zeta$ & -- & wall damping ratio parameter \\
\hline$\eta$ & $\begin{array}{l}-- \\
--\end{array}$ & $\begin{array}{l}\text { non-dimensional wall velocity } \\
\text { efficiency }\end{array}$ \\
\hline$\Lambda$ & -- & wall motion component of eigen vector \\
\hline$v$ & -- & kinetic viscosity \\
\hline$\pi$ & -- & pressure ratio \\
\hline$\rho$ & -- & density \\
\hline$\tau$ & -- & non-dimensional time \\
\hline$\phi$ & -- & mass flow coefficient \\
\hline$\Phi$ & -- & mass flow component of eigenvector \\
\hline$\chi$ & -- & slip factor \\
\hline$\Psi$ & -- & pressure rise coefficient \\
\hline$\omega$ & -- & frequency \\
\hline$\Theta$ & -- & wall motion component of eigenvector \\
\hline
\end{tabular}




\begin{tabular}{|c|c|c|}
\hline \multicolumn{3}{|c|}{ Subscripts } \\
\hline $\operatorname{adj}$ & -- & adjusted \\
\hline aux & -- & auxiliary \\
\hline c & -- & compressor \\
\hline cor & -- & corrected \\
\hline $\mathrm{h}$ & -- & Helmholtz \\
\hline $\mathrm{i}$ & -- & summation index \\
\hline in & -- & inlet \\
\hline meas & -- & measured \\
\hline $\mathrm{p}$ & -- & plenum \\
\hline $\mathrm{t}$ & -- & $\begin{array}{l}\text { throttle } \\
\text { tip of impeller exit }\end{array}$ \\
\hline th & -- & thermal \\
\hline $\mathrm{x}$ & -- & axial \\
\hline & -- & distance from leading edge \\
\hline 0 & -- & ambient conditions \\
\hline 1 & -- & compressor exit \\
\hline 2 & -- & plenum exit \\
\hline Qpera & ors & \\
\hline$\delta()$ & -- & perturbation quantity \\
\hline$\Delta()$ & - & difference \\
\hline$\partial()$ & & \\
\hline$\overline{\partial()}$ & -- & partial derivative \\
\hline () & -- & time derivative \\
\hline$\overline{()}$ & -- & time averaged \\
\hline()$^{\prime}$ & -- & fluctuation \\
\hline$\widetilde{()}$ & -- & Fourier Transform \\
\hline
\end{tabular}




\section{INTRODUCTION}

\subsection{DESCRIPTION OF THE PROBLEM}

The operating range of turbomachinery compression systems is generally limited by the onset of fluid dynamic instabilities which can take one of two forms: surge or rotating stall. Surge is a self-excited, one dimensional, instability characterized by oscillations in areaaveraged mass flow and pressure rise. Rotating stall is a localized, two- or three-dimensional instability characterized by circumferential mass flow and pressure distortions rotating around the compressor face. Although rotating stall can occur in both axial and centrifugal compression systems, it has a much more pronounced effect on performance in axial machines than in centrifugal machines, and surge appears to be the more important instability in centrifugal compression systems 1 . The work in this thesis addresses surge suppression, which is viewed as an intial step in the development of systematic procedures for dynamic control of turbomachinery instabilities.

Surge can be further defined according to the amplitude of the oscillations. Mild surge refers to small amplitude oscillations with a frequency near the Helmholtz resonator frequency of the compression system. Deep surge is characterized by large amplitude oscillations with periods of reversed mass flow. This type of oscillation can cause reduced performance and efficiency of the turbomachine, and even in some cases, failure due to the large unsteady aerodynamic forces on the blades 2 .

To avoid surge, the compression system must be operated away from the "surge line", which denotes the boundary between stable and unstable operation. Operating the compressor to the right of this line, on the negatively sloped part of the compressor speedlines ensures stable operation. A certain distance, or margin, from this line is necessary to prevent the compressor from crossing the surge line due to external disturbances. However, operating a 'safe distance' from the surge line can result in a performance and efficiency penalty since peak performance and efficiency often occur near the surge line. ${ }^{3}$ 
The goal of this research is to develop methods to suppress surge and extend the stable operating range by modifying the compression system dynamics. This would allow the compressor to operate in previously unstable regions of the compressor map as shown is Figure 1.1. The experimental phase of the research is limited to centrifugal compression systems, although the analytical model could be applied to axial compression systems as well.

\subsection{BACKGROUND}

\section{a. Compression System Model}

Typical compression systems consist of a compressor, a discharge chamber, a throttle mechanism, and the associated ducting. For low frequency oscillations, the inertial, resistive, and compliant properties of the compression system can be modelled as lumped parameters. This type of dynamic model has been developed and investigated by many researchers. 1, 2, 4 In this approach, the fluid in the inlet ducting serves as the system's inertance, the volume in the plenum serves as the system's compliance, and the compressor and throttle, modelled as actuator disks, serve as the system's dampers (positive or negative). A schematic of the basic compression system is shown in Figure 1.2. Although the model ignores details of the local fluid dynamics of the flow, it has been shown to accurately model many aspects of the compression system dynamics.

Previous authors have used this model to predict system stability at a given operating point and to investigate surge dynamics in centrifugal compression systems. $1,2,4$ The instabilities observed are most often dynamic in nature; surge occurs when the compressor feeds more energy into disturbances than the throttle can dissipate. The result is an oscillatory disturbance that grows exponentially until nonlinearities force the oscillation into a limit cycle (surge cycle).

For the compressor to feed energy into disturbances, the slope of the compressor's pressure rise versus mass flow characteristic must be positive. 5,6 The magnitude of the positive compressor slope required for surge is determined by system damping characteristics, 
but , for typical centrifugal compression systems, only a slightly positive slope is necessary. For this reason, the surge line generally occurs near the peak of the compressor characteristic. 7

\section{b. Previous Work on Surge Suppression}

There have been several investigations of surge suppression, all in recent years, using closed loop active control. In 1987, Huang 8(University of Cambridge), designed a low speed experimental facility which sucessfully suppressed surge using a movable plenum wall driven by a signal proportional to the unsteady plenum pressure. Pinsley ${ }^{9}$ (Gas Turbine Lab, MIT), actively stabilized surge at high speed using a variable area throttle valve, also driven by a signal proportional to the unsteady plenum pressure. Each of these authors demonstrated that although fully developed surge is highly nonlinear, surge can be suppressed in the linear regime before it grows into a large amplitude disturbance by modification of system dynamics through closed looped control. In addition, the Helmholtz resonator model accurately predicted the effect of the control system on the surge dynamics. Because the control system responded to small amplitude disturbances to supress surge, very little control power was required compared to the steady state output of the compression system 8,9 .

There has also been some previous work on passively stabilizing surge. In these methods the system dynamics are modified such that the compression system becomes inherently stable, requiring no external input. Passive control of surge is similar in concept to the mass balancing techniques used to eliminate flutter by modifying the interaction between the wing and the airstream. Chen 10 conducted a limited analytical investigation of passive control schemes. He found that a properly tuned variable area throttle valve, modelled as a mass-spring-damper driven by plenum pressure perturbations, could have a slightly stabilizing effect. In addition, he showed that a flexible plenum wall with no damping would be destabilizing. 


\subsection{PRESENT WORK}

The work in this thesis is directed at developing methods to suppress surge in centrifugal compression systems using tailored structural feedback. Although the main focus of the analysis and experimental investigation is on the flexible plenum wall method, several other passive control strategies are developed analytically in Appendix B. The unifying theme of each is that the system dynamics are modified to provide additional unsteady energy dissipation through aerodynamic or structural dynamic feedback.

With the flexible plenum wall, the wall is modelled as a mass-spring-damper system driven by unsteady pressure perturbations in the plenum, and coupled to the compression system dynamics A schematic of the flexible plenum wall system is shown in Figure 1.3.

It will be shown that an appropriately tailored flexible plenum wall can significantly extend the stable operating range of a compression system. The aeroelastic coupling between the wall and the basic compression system allows the damper on the flexible wall to dissipate unsteady energy from flow disturbances, thus suppressing surge and extending the stable operating range. The degree of surge suppression achievable is dependent on matching the structural dynamics to the system fluid dynamics. A set of non-dimensional control parameters which govern the interaction between the compression system and the wall are presented and their influence is investigated.

The research included both analytical and experimental phases. The following questions are addressed:

* What are the dominant parameters which determine the interaction of the flexible plenum wall and the basic compression system?

* How much can the flexible plenum wall increase the predicted stable flow range?

* What is the optimal configuration ?

* What is the physical mechanism responsible for the surge suppression?

* How do non-linearities affect the tailored structure performance? 
Based on the results of the initial analytical studies, experiments were designed to assess the validity of the model In particular, are the major assumptions correct and is the actual performance of the system accurately predicted? 


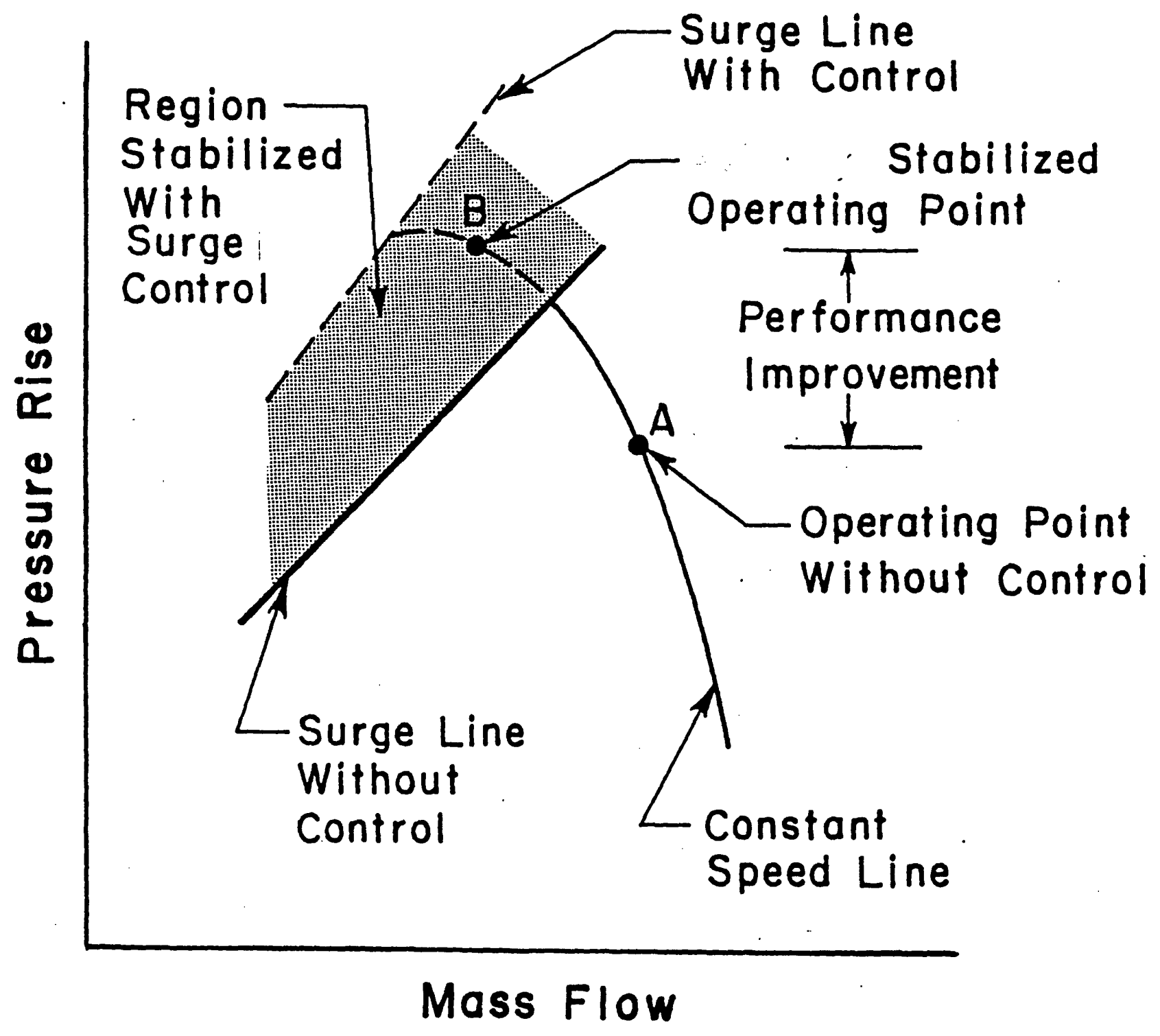

Figure 1.1: Extension of Operating Range with Dynamic Surge Control 


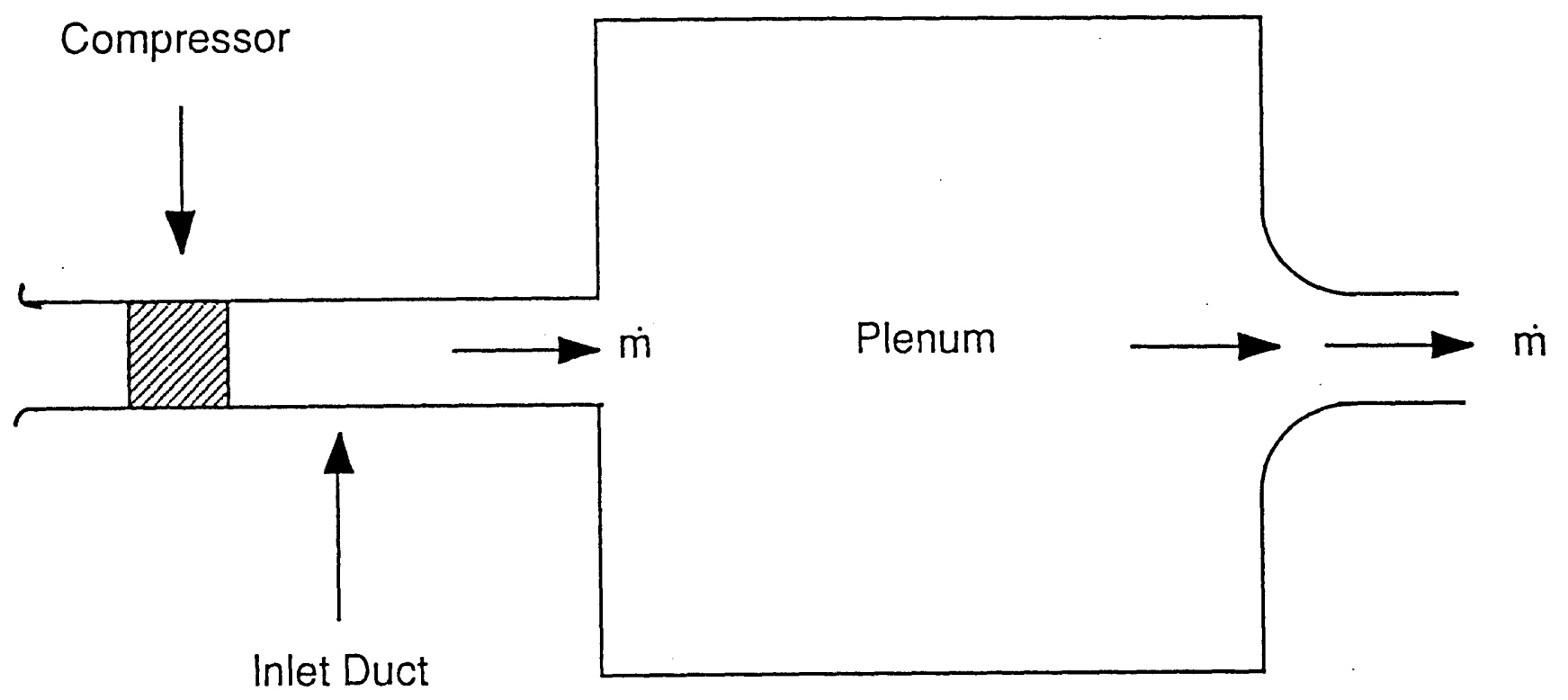

Figure 1.2: Schematic of Basic Compression System 


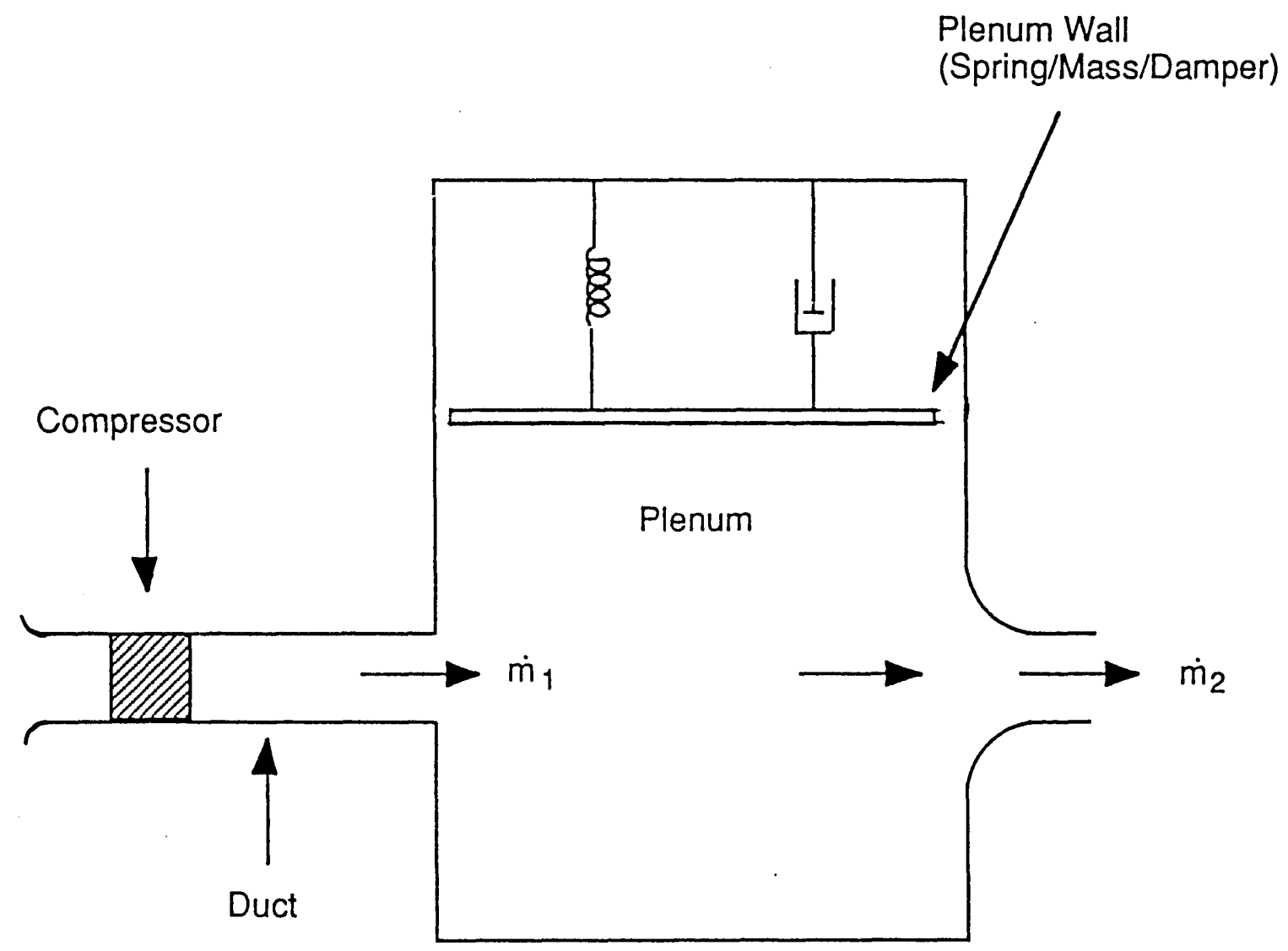

Figure 1.3: Schematic of Compression System with Tailored Structure (Flexible Plenum Wall) 


\section{ANALYSIS}

\subsection{INTRODUCTION}

The basic compression system model has been used by other authors to investigate surge $1,2,4$. The system equations are derived by applying conservation of mass, momentum and entropy to a lumped parameter model. As stated, system inertia is lumped into the fluid in the inlet ducting, system compliance is lumped into the fluid in the volume of the plenum, and system resistance (positive or negative) is lumped into the compressor and the throttle. A schematic of the fixed wall, basic compression system is shown in Fig 1.2 .

The basic system was then modified to include the tailored structure, a flexible plenum wall as shown in Fig 1.3. In this configuration, the wall, (mass-spring-damper), responds to perturbations in plenum pressure. The resulting model for the transient behavior is a set of four, non-linear, coupled differential equations.

To predict the system stability, the equations of motion were linearized. This leads to an eigenvalue problem which links the system stability to the non-dimensional system Using this, optimization studies were performed in parameter space.

In addition to the linearized analysis, the non-linear system equations were integrated in time numerically to study finite amplitude behavior. This non-linear analysis enabled the study of system behavior in surge.

\subsection{COMPRESSION SYSTEM MODELLING}

a. Assumptions

In deriving the equations of motion for the system, the following assumptions were made.

1) Flow in the inlet ducting is 1-D, incompressible, inviscid, and unsteady

2) Plenum pressure is spatially uniform

3) Fluid in the plenum behaves isentropically

4) Fluid inertia in the throttle is negligible 
5) Compressor follows a quasi-steady characteristic

6) Wheel speed is constant

7) Throttle characteristic is parabolic

Justification of these assumptions is well documented by other authors $1,4,5,6$ with the exception of the constant wheel speed and quasi-steady compressor performance assumptions. The constant wheel speed assumption is based on the analysis by Fink ${ }^{1}$ in which he investigated the effects of a constant torque, variable speed rotor on a compression system stability. Fink showed that variable speed always has a stabilizing effect, and that the degree of stabilization is determined by a parameter which relates the aerodynamic torque on the rotor to the wheel's rotational inertia. This parameter, defined as:

$$
A=\frac{2 \rho_{o} L_{c} A_{\text {in }} R_{T}^{2}}{I}
$$

affects linear stability only for $\mathrm{A}>0.5$. For the present system, the A-parameter is an order of magnitude below this critical value. Therefore, variations in wheel speed were neglected.

Many authors have investigated the instantaneous compressor characteristic during the onset of surge 1,8,11. It is has been speculated that the unsteadiness in the compressor during the onset of surge causes the compressor to vary from its steady state characteristic, but , the degree to which the unsteady aerodynamics modify the compressor characteristic has not been quantified. Fink ${ }^{1}$ showed that the instantaneous compressor characteristic is slightly flatter than the quasi-steady characteristics due to an unsteady aerodynamic lag term which is on the order of compressor flow through time. It was found, however, that this lag term had little effect on system stability. Therefore, the quasi-steady assumption was used in the analysis and its accuracy assessed later with experimental results.

b. Equations for the System Dynamic Performance

The governing differential equations for the compression system with flexible plenum wall are: conservation of momentum in the inlet duct, conservation of mass in the plenum, and 
Newton's second law applied to the flexible wall plus knowledge of the quasi-steady compressor and throttle characteristics. A derivation of the equations of motion is given in Appendix A and we merely list below the set of non-linear equations governing the compression system dynamics.

$$
\begin{array}{ll}
\text { (compressor characteristic) } & \mathrm{P}_{0}+\Delta \mathrm{P}_{\mathrm{c}}\left(\dot{\mathrm{m}}_{1}\right)=\mathrm{P}_{1} \\
\text { (inlet duct momentum) } & \left(\mathrm{P}_{1}-\mathrm{P}_{\mathrm{p}}\right) \mathrm{A}_{\mathrm{in}}=\frac{\mathrm{d}\left(\rho_{0} \mathrm{~A}_{\mathrm{in}} \mathrm{L}_{\mathrm{c}} \mathrm{U}\right)}{\mathrm{dt}} \\
\text { (plenum continuity) } & \dot{\mathrm{m}}_{1}-\dot{\mathrm{m}}_{2}=\frac{\mathrm{d}\left(\rho_{\mathrm{p}} \mathrm{V}_{\mathrm{p}}\right)}{\mathrm{dt}} \\
\text { (throttle characteristic) } & \mathrm{P}_{\mathrm{p}}-\Delta \mathrm{P}_{\mathrm{t}}\left(\dot{\mathrm{m}}_{2}\right)=\mathrm{P}_{0} \\
& \\
\text { (wall dynamics) } & \mathrm{m} \ddot{\mathrm{q}}+\mathrm{cq}+\mathrm{kq}=\left(\mathrm{P}_{\mathrm{p}}-\mathrm{P}_{\mathrm{o}}\right) \mathrm{A}_{\mathrm{p}}
\end{array}
$$

Linearizing and non-dimensionalizing the equations of motion as shown in appendix A, and assuming isentropic compression in the plenum, yields the following equations that describe the linear stability of the system.

(inlet. duct momentum) $\frac{\partial \Psi}{\partial \phi} \delta \phi_{1}-\delta \Psi=\frac{1}{B} \delta \dot{\phi}_{1}$

$$
\text { (plenum continuity) } \delta \phi_{1}-\delta \phi_{2}=\mathrm{B} \frac{\partial \Psi}{\partial \tau}+\frac{\rho_{\mathrm{p}}}{\rho_{0}} \frac{\mathrm{B}}{\mathrm{M}_{\mathrm{t}}^{2}} \frac{\partial \mathrm{v}}{\partial \tau}
$$

$$
\text { (throttle characteristic) } \quad \delta \Psi=\frac{\rho_{0}}{\rho_{\mathrm{t}}} \frac{\phi}{\widehat{\mathrm{A}}_{t}^{2}} \delta \phi_{2}
$$

$$
\text { (wall dynamics) } \quad \frac{\mathrm{d} \delta \mathrm{v}}{\mathrm{d} \tau}+2 \sqrt{\frac{\mathrm{P}_{0} \rho_{\mathrm{p}}}{\mathrm{P}_{\mathrm{p}} \rho_{0}}} \zeta \mathrm{Q} \frac{\mathrm{d} \delta \mathrm{v}}{\mathrm{d} \tau}+\frac{\rho_{\mathrm{p}}}{\rho_{0}} \mathrm{Q}^{2} \delta \mathrm{v}=\mathrm{W} \mathrm{B}^{2} \delta \Psi
$$


where $\Psi, \phi$ and $v$ are defined as:

(pressure coefficient)

(mass flow coefficient)

(non-dimensional wall motion)

$$
\Psi=\frac{P_{p}-P_{0}}{\rho_{0} U^{2}}
$$

and $\delta \Psi, \delta \phi_{1}, \delta \phi_{2}$, and $\delta \mathrm{v}$ are the small perturbation quantities in pressure rise coefficient, inlet mass flow coefficient, exit mass flow coefficient, and non-dimensional wall motion, respectively.

Non-dimensional time is defined in terms of the Helmholtz frequency as:

$$
\tau=\omega_{h} t
$$

The non-dimensional parameters appearing in the linearized equations of motion can be classified as compression system parameters and structural control parameters. The parameter $\partial \Psi / \partial \phi$ is the non-dimensional slope of the compressor characteristic and is linked directly to surge. In the negatively sloped regions of the compressor map, both the throttle and the compressor serve to damp out flow disturbances. In the positively sloped regions, the compressor adds energy to disturbances while the throttle continues to dissipate unsteady energy. The flow through the system becomes unstable when the compressor adds more energy into disturbances than the throttle can extract 6 .

The B-parameter is the major system parameter which determines the surge dynamics of the compression system $1,2,5$. The B-parameter is defined in this thesis* as:

\footnotetext{
*Note: The definitions of the B parameter and the pressure coefficient, $\Psi$, do not contain a factor of 2 , differing from common practice.
} 


$$
B=\frac{U}{\omega_{h} L_{c}}=\frac{\rho_{0} U^{2} A_{\text {in }}}{\rho_{0} A_{\text {in }} L_{c} \omega_{h} U}
$$

It can be viewed as the ratio of pressure forces to inertial forces present in the Helmholtz resonator oscillations. The magnitude of the positive compressor slope required for disturbances to grow is determined by the B-parameter, and the B-parameter can thus be viewed as providing a measure of the coupling between mass flow oscillations through the compressor and through the throttle. The larger the B-parameter, the more isolated the throttle is from the compressor, and, therefore, less able to remove energy from flow disturbances. The general trend is that as the B-parameter increases, surge occurs at a smaller positive compressor slope.

The Helmholtz frequency of the compression system is defined as:

$$
\omega_{\mathrm{h}}=\mathrm{a}_{\mathrm{p}} \sqrt{\frac{\mathrm{A}_{\text {in }}}{\mathrm{V}_{\mathrm{p}} \mathrm{L}_{\mathrm{c}}}}
$$

where: $\quad \mathrm{L}_{c}$ is the effective length of the inlet duct,

$A_{\text {in }}$ is area of the duct,

$V_{p}$ is the plenum volume

$a_{p}$ is the speed of sound within the plenum.

The non-dimensional pressure rise and mass flow coefficients, $\Psi$ and $\phi$ define the steady state operating conditions.

The tip Mach number is based on plenum conditions and defined as:

$$
\mathrm{M}_{\mathrm{t}}=\frac{\mathrm{U}}{\mathrm{a}_{\mathrm{p}}} \quad \text { (tip Mach number) }
$$

enters the non-dimensional equations of motion as a measure of the effect of wall motion on the plenum continuity equation, but, it does not appear explicitly in the fixed wall model. The tip Mach number affects the coupling of wall motion to the compression system dynamics by determining the degree to which plenum pressure responds to wall motion. The pressure and mass flow fluctuations scale as a functions of tip Mach number (as $\mathrm{M}_{\mathrm{t}}{ }^{2}$ for low speeds), but 
the wall motion does not. The Mach number is needed in the equations of motion to include this effect, rather than as an explicit representation of the importance of compressibility in the system dynamic model. The larger the Mach number, the smaller the effect a given nondimensional wall motion will have on the non-dimensional mass flow and pressure perturbations in the system. In fact, the linear analysis predicts in the limit as $M_{t}=\infty$, the basic compression system becomes completely uncoupled from the wall motion so that the flexible plenum wall does not affect system stability.

The control parameters, $\mathrm{W}, \zeta$, and $\mathrm{Q}$, determine the wall's dynamic characteristics relative to the basic compression system dynamics. $W$ is an aeroelastic coupling parameter defined as

$$
\text { (aeroelastic coupling parameter) } \quad \mathrm{W}=\frac{\rho_{0} A_{p}^{2} L_{c}^{2}}{m V_{p}}
$$

This parameter determines the degree to which wall responds to the pressure fluctuations in the plenum. The larger the W-parameter, the more the wall responds to perturbations in plenum pressure.

$\zeta$ is the critical damping ratio of the plenum wall mass-spring-damper system, corrected to remain independent of compressor operating conditions. This is necessary since the aerodynamic spring constant , and hence, the wall natural frequency, varies with plenum pressure.

(a damping ratio)

$$
\zeta=\frac{c}{2 m \omega_{p}} \sqrt{\frac{P_{p}}{P_{0}}}
$$

$Q$ defines the ratio of natural frequencies between the wall mass-spring-damper system and the compression system, also corrected so that it is independent of compressor operating point.

(frequency ratio)

$$
\mathrm{Q}=\frac{\omega_{\mathrm{p}}}{\omega_{\mathrm{H}}} \sqrt{\frac{\rho_{0}}{\rho_{\mathrm{p}}}}
$$




\subsection{LINEAR STABILITY ANALYSIS}

A linearized stability analysis was used to examine the effect of structural control parameters on system stability for a given set of operating conditions. The goal was to shift the surge line as far towards low flow as possible for a given set of aerodynamic system parameters. As mentioned, the dominant stability parameters for the compression system are the B-parameter and the quasi-steady compressor slope, $\partial \Psi / \partial \phi$. The quasi-steady compressor characteristic gives a direct relationship between the compressor slope and (steady state) mass flow so that the latter can also be used to characterize the system operating point. This substitution was made to illustrate in a conventional manner the shift in the surge line for a given compressor, but it should be noted that the maximum slope prior to the onset of instability is a more appropriate measure of the effectiveness of a control strategy.

The stability matrix for the system is shown below.

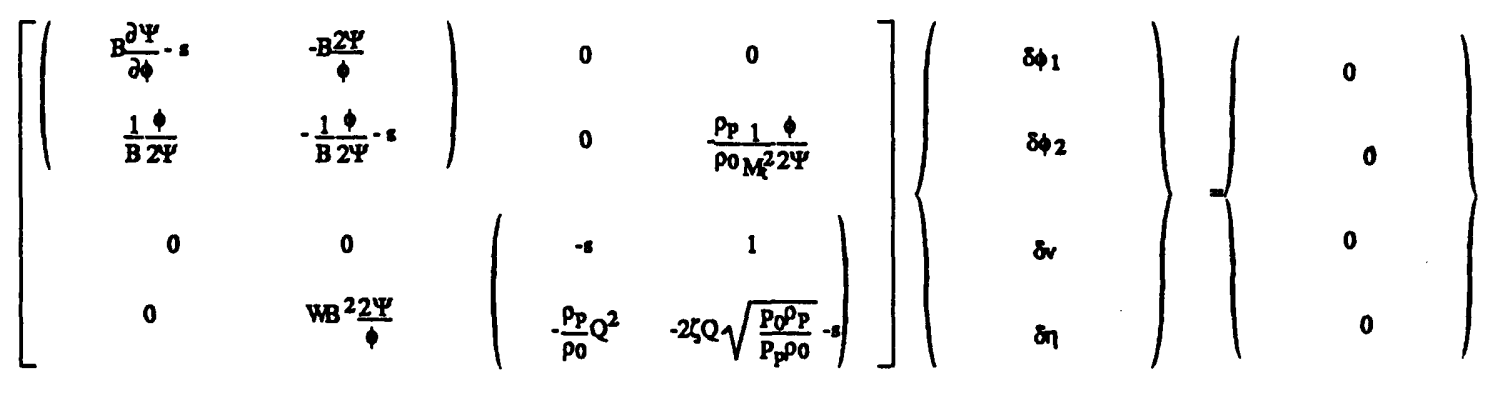

This essentially involves two coupled, dynamic systems; the compression system involving $\delta \phi_{1}$ and $\delta \phi_{2}$ and the wall system involving $\delta v$ and $\delta \eta$. The terms associated with the uncoupled fixed wall compression system and the flexible plenum wall dynamics are located on the tri-diagonal of the stability matrix and are shown in the dotted brackets. The two non-zero terms located off the tri-diagonal are the aeroelastic coupling terms.

Solving the eigenvalue problem as a function of $\mathrm{B}, \mathrm{M}_{\mathrm{t}}, \phi, \mathrm{W}, \zeta$, and $\mathrm{Q}$ and using the experimentally determined close-coupled characteristics of Pinsley ${ }^{9}$ provides a method to predict the movement of the surge line for various system and control parameters. Close- 
coupled characteristics refer here to compressor characteristics measured with a system having a very low value of $B$, and thus much resistant to surge. This allows the quasi-steady performance to be measured beyond the surge line for systems with higher B-parameters. The close-coupled $70 \mathrm{~K}$ rpm speedline used in the stability analysis is shown in Figure 2.1. Steady state plenum temperature was determined from the pressure ratio and a nominal $65 \%$ adiabatic efficiency; the effect on the results for any reasonable assumption for the efficiency is negligible.

\section{a. Fixed Wall Compression System}

The stability of the fixed wall system can be predicted using the above stability matrix in the limit of either $W=0$ or $Q=\infty$. Either has the effect of making the wall appear massive and the spring constant stiff,or essentially rigid. For the fixed wall system, the stability criteria, developed in Appendix A, is given as:

$$
\frac{\partial \Psi}{\partial \phi}<\frac{1}{B^{2}} \frac{\phi}{2 \Psi}
$$

The maximum stable compressor slope is determined by the B-parameter and steady state operating conditions.

The root locus plot for a fixed wall system with $B=1.0$, plotted as a function of mass flow, is shown in Figure 2.2. The root locus plot is based on a 3rd degree polynomial curve fit of the measured 70K speedline shown in Figure 2.1.The abscissa and ordinate of the root locus plots are non-dimensionalized by the system Helmholtz resonator frequency. The roots are plotted for mass flow coefficients ranging from .175 to .07 in increments of .005 .As the mass flow decreases, corresponding to increasing the compressor slope, the poles are driven from the left half plane (stable) to the right half plane (unstable), with the imaginary axis defining the neutral stability point. Also, as shown by the root locus plots, the Helmholtz frequency is predicted to be a good estimate of the system natural frequency over the range of 
mass flow coefficients. The system behaves like a damped (positive or negative) second order oscillator. The neutral stability point for the fixed wall system $(\phi=.116)$, shown on the compressor characteristic in Figure 2.1, occurs near the peak of the compressor characteristic which is located at $\phi=0.120$.

\section{b. Flexible Plenum Wall System}

The introduction of a flexible plenum wall introduces a second mode of oscillation to the compression system. In the limiting case, where the aeroelastic coupling is small, the frequencies of the structural and fluid modes are far apart. However, in the range of parameters for which the wall is effective in suppressing surge, the modes show strong fluid structural interaction and cannot be readily linked to either of the two modes of oscillations in the absence of the coupling.

To optimize the flexible wall compression system performance, a parametric study was performed. In most compression systems, the physical dimensions of the machine are independent of compressor operating conditions. However, for compression systems with a fixed inlet duct length and plenum volume, as was the case here, the tip Mach number and Bparameter are not independent, but scale directly with tip speed. The relationship between the Mach number and the B-parameter depends on the Helmholtz frequency and the inlet duct length. The relation between these two parameters used in this study is based on selecting values for the dimensions of the compression system which were both typical of modern compression machines and convenient from an experimental view point. This relation is shown in Figure 2.3.

The result of the parameter search showed that the flexible wall performance is optimized over the range of B-parameters and Mach numbers with the following control parameters: $W=11, \zeta=1.5$, and $Q=.51$. The root locus plot of the two mode system $(B=1.0$, $\mathrm{Mt}=.4$ ) is shown in Figure 2.4. The 70K compressor speed characteristic shown in Figure 2.1 was used here also. The Helmholtz frequency is again close to the characteristic frequency 
of the system, but now the system behaves as two second order oscillators and the neutral stability point occurs well past the peak of the characteristic in the positively sloped region, as shown in Figure 2.4. The frequency of the mode that first becomes unstable is approximately 0.9 of the fixed wall Helmholtz frequency.

Away from instability (high mass flow), the flexible wall system has one oscillatory mode and one non-oscillatory mode; these are close to the Helmholtz mode and an overcritically damped wall mode. Near instability, the two modes show more fluid-structure coupling and both become oscillatory.

As noted previously, the maximum controllable slope before deep surge is a more appropriate measure of the control system performance than the minimum mass flow before deep surge. This is demonstrated in Figure 2.5, where the maximum controllable slope for the optimized and fixed wall configurations is shown to vary little over a large range of mass flow coefficients. The maximum controllable slope in the figure is calculated by increasing the nondimensional compressor slope at a given operating point until the system developed a positive real root. Figure 2.5 shows that the steady state mass flow coefficient (by itself, for a given slope) is not a dominant stability parameter for the optimized flexible wall system, however, it is an important parameter for the fixed wall compression system as indicated by equation (2.21).

Determining the maximum controllable slope versus the B-parameter, (as suggested by Pinsley ), is much more useful. The larger the positive compressor slope before instability or surge, the more effective the control system. The maximum controllable slope versus Bparameter for the uncontrolled system and the optimized flexible wall system is shown in Figure 2.6. As shown, the maximum controllable slope is shown to be a function of the Bparameter for each system,with the optimized system capable of operating at much larger positive compressor slopes than the fixed wall system.

Plotting maximum controllable slope versus each of the control parameters about the optimized configuration in parameter space demonstrates that the result of the parameter study 
is at least a local maximum in parameter space. Figure 2.7 shows the maximum controllable slope versus $W, \zeta, Q$ varied respectively. The optimized values are indicated by arrows. As shown, any substantial changes in the structural control parameters away from the optimized configuration destabilizes the system. However, the linear analysis predicts the stabilization to be fairly insensitive to small changes in the control system parameters. The isolated effect of the B-parameter and the tip Mach number for system with a fixed set of control parameters is shown in Figure 2.8A, where one parameter is held constant and the other varied. Although this is not physically practical study since it would require varying the dimensions of the compression system with the operating point, it does demonstrate that the tip Mach number is an important parameter governing the stability of the flexible wall system over this range of parameters.

The parameter study yielded a system optimized over a range of Mach numbers $(0.4$ to 0.7). Over this range, which was selected because it is representative of the conditions in the intended experimental test rig, the aeroelastic coupling parameter was held constant, maintaining the independence of compression system geometry with operating point. The control system could have been optimized for a different range of Mach numbers, and the same range of B-parameters, by modifying the system Helmholtz frequency. Changing the range of design Mach numbers would require re-optimization of the system's control parameters, mainly modifying the aeroelastic control parameter, W. Figure $2.8 \mathrm{~B}$ demonstrates this by plotting the effect of Mach number on the stability of the optimized flexible wall compression system with a modified aeroelastic coupling parameter. As shown, for the system with the smaller aeroelastic coupling parameter $(W=.02)$, the maximum control occurs at a Mach number of 0.2 . For the system with $\mathrm{W}=.11$, the maximum control occurs at Mach number = 0.6. Although the Mach number for which the system is optimized varies between the two systems, the maximum controllable slope remains fairly constant. 


\subsection{ENERGY BALANCE}

The idea of an optimized configuration introduces the question of identifying the physical limitations on the maximum effectiveness of a flexible plenum control system. To address this issue, one must examine the physical mechanisms which stabilize the system. The dynamics of each mode of the flexible plenum wall compression system can be analyzed individually 12,13. From an energy viewpoint, the system is said to be unstable when more energy is fed into any mode of oscillation over a cycle than is removed.

If the net energy input over a cycle is positive, the oscillation grows until non-linearities force the system into a limit cycle or surge. Therefore, if one considers the net energy input to a mode over a cycle, neutral stability will correspond to zero net energy input. Using the orthogonality of the modes, it is only necessary to consider one mode at a time. If any mode is unstable,the system is unstable.

First, consider the fixed wall system. This model contains only one possible mode of oscillation and only two mechanisms for energy input and dissipation, flow through the compressor and throttle, respectively. The relative unsteady energy flows over a cycle can be calculated for neutrally stable modes with pure imaginary roots. Using the eigenvector of the nuetrally stable mode, the energy input by the compressor and dissipated by the throttle is calculated using the following relations.

The work transferred to the fluid by the compressor over a cycle can be expressed as:

$$
\mathrm{W}_{c}=\int_{t=0}^{t=2 \pi / \omega} \text { power dt }=\int_{1=0}^{t=2 \pi / \omega} \frac{\Delta \mathrm{P}}{\rho_{0}} \dot{\mathrm{m}}_{1} \mathrm{dt}
$$

Applying linearized small perturbation theory, the unsteady work can be expressed as: 


$$
W_{c}=\rho_{0} U^{3} A_{i n} \int_{t=0}^{t=2 \pi / \omega}\left(\Psi+\frac{\partial \Psi}{\partial \phi} \delta \phi_{1}\right) \delta \phi_{1} d t
$$

where: $\delta \phi_{1}=\Phi_{1} \mathrm{e}^{\mathrm{i} \omega \mathrm{t}}$; as given by the eigenvector of the neutrally stable mode. Performing the integration over an cycle yields:

$$
\mathrm{W}_{\mathrm{c}}=\frac{\pi \rho_{0} \mathrm{U}^{3} \mathrm{~A}_{\mathrm{in}}}{\omega} \frac{\partial \Psi}{\partial \phi} \Phi_{1}^{2}
$$

Similarly, for the throttle:

$$
\mathrm{W}_{\mathrm{t}}=\frac{\pi \rho_{0} \mathrm{U}^{3} \mathrm{~A}_{\text {in }}}{\omega} \frac{2 \Psi}{\phi} \Phi_{2}^{2}
$$

where $\Phi_{1}$ and $\Phi_{2}$, are components of the neutrally stable eigenvector and $\omega$ is the frequency of the neutrally stable mode. (The energy expressions given above, assume that density remains constant through the compression system.) At the neutral stability point the relative unsteady energy fed into the system by the compressor balances the unsteady energy dissipated by the throttle 10 .

A more interesting analysis results from looking at energy balances between the compressor, throttle, and the flexible wall in the controlled system. The relative unsteady energy dissipated by the flexible plenum wall for oscillations in the neutrally stable mode is given by:

$$
\mathrm{W}_{\text {wall }}=\int_{\text {cyde }} \mathrm{Fdq}=\mathrm{c} \int_{0}^{2 \pi / \omega} \dot{\mathrm{q}}^{2} \mathrm{dt}
$$

where $\dot{q}=i \omega \Theta \mathrm{e}^{\mathrm{i} \omega \mathrm{t}}$ and $\Theta$ is the wall motion component in the eigenvector of the 
neutrally stable mode. Performing the integration yields:

$$
\mathrm{W}_{\mathrm{wall}}=\pi \mathrm{c} \omega \Theta^{2}
$$

Figure 2.9 shows the relative energies input and dissipated over a cycle at the neutral stability point as function of the B-parameter for the optimized system. The energies calculated were based on the eigenvector of the neutrally stable mode. The flexible wall is the dominant unsteady energy dissipator for the tuned wall, dissipating approximately 10 - 15 times as much as the throttle over a large range of B-parameters. Also, the energy analysis shows that main stabilizing mechanism of the flexible plenum wall compression system is the plenum wall itself, rather than modification of the system dynamics to cause the throttle to dissipate more unsteady energy, as in Pinsley's experiments9.

For the wall to remove unsteady energy input by the compressor, the two components must have sufficient aeroelastic coupling to transfer the energy from the pressure fluctuations to the damper where it is dissipated. It is in this coupling where the limit to the effectiveness of the control scheme lies. To investigate the limitations of the method for suppressing surge, the flexible plenum wall dynamics can be uncoupled from the rest of the compression system and the aeroelastic coupling term can be viewed as an external forcing function. The format is shown below:

$$
\left[\begin{array}{cc}
-s & 1 \\
\frac{\rho_{\mathrm{p}}}{\rho_{0}} \mathrm{Q}^{2} & -2 \zeta \mathrm{Q} \sqrt{\frac{\mathrm{P}_{0} \rho_{\mathrm{p}}}{\mathrm{P}_{\mathrm{p}} \rho_{0}}}-\mathrm{s}
\end{array}\right]\left\{\begin{array}{c}
\delta \mathrm{v} \\
\delta \eta \sum_{\eta}^{?} ?
\end{array}\right\}=\left\{\begin{array}{c}
0 \\
\mathrm{WB}^{2} \frac{2 \Psi}{\phi} \delta \phi_{2}
\end{array}\right\}
$$

The magnitude of the external forcing function is dictated by the rest of the system dynamics through the solution to the complete eigenvalue problem.

For the purpose of the study, it was first necessary to characterize the energy dissipation in a simple mass-spring-damper system driven by a constant sinusoidal forcing 
function. The energy dissipation by the dashpot, the only non-conservative component in the system, increases with decreasing mass, if the forcing function, damping constant and frequency parameter are held constant. This indicates that the less massive the wall, the more effective it would be in suppressing surge. However, in the coupled system. the effective forcing function is dependent on wall motion through the system dynamics. When the system dynamics are considered, the aeroelastic coupling (or forcing function acting on the wall) decreases with decreasing plenum wall mass $\left(M_{t}=\right.$ constant). The amount of energy dissipation in the wall is limited by the amount which can be transferred into the wall through the coupling of the two systems. Therefore, unsteady energy dissipation by the wall has a finite limit or maximum at some optimized condition. The limit on energy dissipation in the dashpot limits the maximum effectiveness of this device.

\subsection{NON-LINEAR ANALYSIS}

The results of the linear stability analysis yielded a set of optimized, non-dimensional control parameters. Large increases in the maximum controllable slope and, hence, large increases in the stable flow range were predicted These predictions, however, were based soley on a linearized model of the flexible wall compression system.

A non-linear, numerical, time marching integration of the equations of motion was also performed. The equations of motion (Eq 2.29 - 2.34) were integrated using the Newmark time-averaged acceleration method. The equations presented below incorporate an aerodynamic spring in the flexible plenum wall dynamics. The spring restoring force is provided by a sealed chamber of air being compressed isentropically, as developed in Chapter 3. The equations of motion in state variable form are shown below.

$$
\text { (inlet momentum) } \quad \ddot{\mathrm{m}}_{1}=\frac{\mathrm{A}_{\text {in }}}{\mathrm{L}_{\mathrm{C}}}\left(\Delta \mathrm{P}_{\mathrm{c}}\left(\dot{\mathrm{m}}_{1}\right)-\mathrm{P}_{\mathrm{p}}+\mathrm{P}_{0}\right)
$$




$$
\begin{array}{ll}
\text { (plenum continuity) } & \dot{\mathrm{P}}_{\mathrm{p}}=\frac{\gamma \mathrm{P}_{\mathrm{p}}}{\rho_{\mathrm{p}} \mathrm{V}_{\mathrm{p}}}\left(\dot{\mathrm{m}}_{1}-\dot{\mathrm{m}}_{2}-\rho_{\mathrm{p}} \mathrm{A}_{\mathrm{p}} \mathrm{x}\right) \\
\text { (wall dynamics) } & \dot{\mathrm{x}}=\frac{\left(\mathrm{A}_{\mathrm{p}}\left(\mathrm{P}_{\mathrm{p}}-\mathrm{P}_{\mathrm{aux}}\right)-\mathrm{cx}\right)}{\mathrm{m}} \\
\text { (definition) } & \dot{\mathrm{q}}=\mathrm{x}
\end{array}
$$

with the following algebraic relations to close the system:

$$
\begin{array}{lc}
\text { (plenum volume) } & \mathrm{V}_{\mathrm{p}}=\overline{\mathrm{V}}_{\mathrm{p}}+\mathrm{A}_{\mathrm{p}} \mathrm{q} \\
\text { (throttle characteristic) } & \Delta \mathrm{P}_{\mathrm{t}}\left(\dot{\mathrm{m}}_{2}\right)=\frac{1}{2} \frac{\dot{\mathrm{m}}_{2}^{2}}{\rho_{\mathrm{t}} \mathrm{A}_{\mathrm{t}}^{2}}
\end{array}
$$

and from the isentropic relation applied to the aerodynmamic spring

$$
\frac{p}{\rho^{\gamma}}=\text { constant }
$$

the following relation can be derived:

(isentropic spring)

$$
P_{a u x}=\bar{P}_{a u x}\left[\frac{\bar{V}_{a u x}}{\bar{V}_{a u x}-A_{p} q}\right]^{\gamma}
$$

Again, knowledge of a compressor constant speed characteristic was needed to perform these calculations. As in the linear analysis, the characteristic shown in Figure 2.1 was used. The non-linear analysis was used mainly in the conceptual design phase of building a demonstrator rig to assess the effects of finite amplitude disturbances and Coulomb friction. The effect of finite amplitude disturbances was analyzed by perturbing the pressure at the inlet of the compressor and calculating the time response of the system. The non-linear analysis 
predicted the system stability to be rather insensitive to the magnitude of inlet disturbances. Figure 2.10 , shows the predicted time history response of a linearly optimized system to a $20 \%$ ambient pressure pulse disturbance at the inlet operating near the linearly predicted stability line $(\phi=.096)$. A $20 \%$ ambient pressure disturbance was much larger than any anticipated ambient or internal disturbance. As shown, the wall motion and pressure perturbations exhibit classical damped harmonic motion. To suppress this disturbance, the maximum non-dimensional wall motion required corresponded to $0.1 \%$ of the plenum volume. The non-linear analysis also showed that introducing wall motion into a fixed wall system undergoing deep surge cycles, could suppress the surge.

An important assumption in the linear analysis was linear wall motion. However, in physical devices Coulomb friction is always present. The presence of Coulomb friction, in a strict sense, invalidates the linearity assumption. However, the degree to which the accuracy of the linear model is affected is a function of the magnitude of the Coulomb friction forces compared to the other, essentially linear, forces in the system ${ }^{12}$. To assess this effect, the equations of motion of the system were modified to include a Coulomb friction term. A friction force of constant magnitude was imposed on the wall in the direction opposite to its motion to model the sliding friction that would be present in an actual design.

An important result of this friction force is that it prevents the wall from responding to disturbances below a certain threshold. Disturbances must grow to a critical amplitude before the wall can respond. Therefore, in the strictest sense, operating points to the left of the natural surge line remain linearly unstable for the actual (non-ideal) control system.

The linear instability resulting from the presence of Coulomb friction, grows into a limit cycle, the amplitude of which is independent of system excitation and depends only on system parameters 12,13 . For a given compression system and set of control parameters, the amplitude of the limit cycle is a function of the non-dimensionalized friction force and the slope of the compressor characteristic. Using the dynamic pressure based on wheel speed and the wall area, the non-dimensional friction force is defined as: 


$$
F=\frac{f}{\rho_{0} U^{2} A_{p}}
$$

The effect of Coulomb friction on surge suppression is demonstrated in Figure 2.11 where the root mean square of the (calculated) limit cycle pressure fluctuations divided by the steady state pressure rise is plotted versus local compressor slope for various non-dimensional friction levels. For a given friction force, the limit cycle has a region in which its amplitude grows very little with increasing slope until the slope reaches a critical value at which the amplitude changes rapidly into the large values associated with deep surge. The maximum value of the compressor slope before deep surge occurs decreases with increasing friction levels. In the limit of increasing Coulomb friction levels, the performance of the flexible plenum wall system approaches that of the fixed wall system.

As an example of limit cycle predicted by the non-linear equations of motion due to a different levels of Coulomb friction, the time response of identical systems with various levels of Coulomb friction to a small disturbance is shown in Figure 2.12. The transients are predicted for the optimized system operating at $B=1.5$ and $\phi=0.105$ on the $70 \mathrm{~K}$ speedline. The amplitude of each limit cycle is shown on Figure 2.11. The disturbance decays in the system without Coulomb friction, but grows into a limit cycle in the systems with Coulomb friction. For the system with a small amount of Coulomb friction $(F=.003)$ the limit cycle is small and approximately sinusiodal (mild surge) with a frequency near the predicted Helmholtz frequency. However, for the system with a larger amount of Coulomb friction $(F=.015)$, the limit cycle is more complex, containing regions of reversed flow (deep surge).

The deep surge boundary for the non-linear system with Coulomb friction and the predicted linear stability boundary for the linearly modeled system agree well in the limit of zero friction. However, as the friction term is increased, the non-linear analysis predicts deep surge at smaller positive compressor slopes. In the limit of large friction forces, the flexible wall system is predicted to encounter deep surge near the linear stability boundary of the fixed wall system. Analysis showed that the flexible plenum wall became unable to suppress surge 
past the rigid wall surge line for non-dimensional friction levels greater than $F=.02$.

The non-linear analysis identified two additional requirements needed to design a test facility. For the wall to suppress finite amplitude disturbances, there should be a minimum range over which the wall responds quasi-linearly. In addition, the analysis shows the importance of minimizing the non-dimensional Coulomb friction in actual applications of the flexible plenum wall control strategy.

\subsection{SUMMARY}

The present analytical investigation of the flexible plenum wall has shown that:

* There is a substantial potential for increases in the maximum controllable slope for a large range of compression systems.

* Maximum controllable slope is a more appropriate measure of a control system's performance than minimum mass flow at instability.

* The use of the flexible plenum wall to suppress surge is predicted to be robust and insensitive to large amplitude disturbances and small changes in control parameters or operating conditions.

*Only a relatively small amount of wall motion is required to suppress large amplitude disturbances, resulting in little power being dissipated by the wall in the controlled regions.

* Coulomb friction in the wall is highly destabilizing, causing the system to go into deep surge at lower compressor slopes than linear theory predicts and causing mild surge to exist over the entire stabilized regions. 
* The damper in the flexible wall, not the throttle, is the dominant unsteady energy dissipator in the optimized flexible plenum wall compression system.

* The maximum effectiveness of a flexible plenum wall to suppress surge is limited by the finite amount of coupling between the wall dynamics and the compression system dynamics.

* The tip Mach number governs the degree to which wall motion modifies the basic compression system dynamics. 


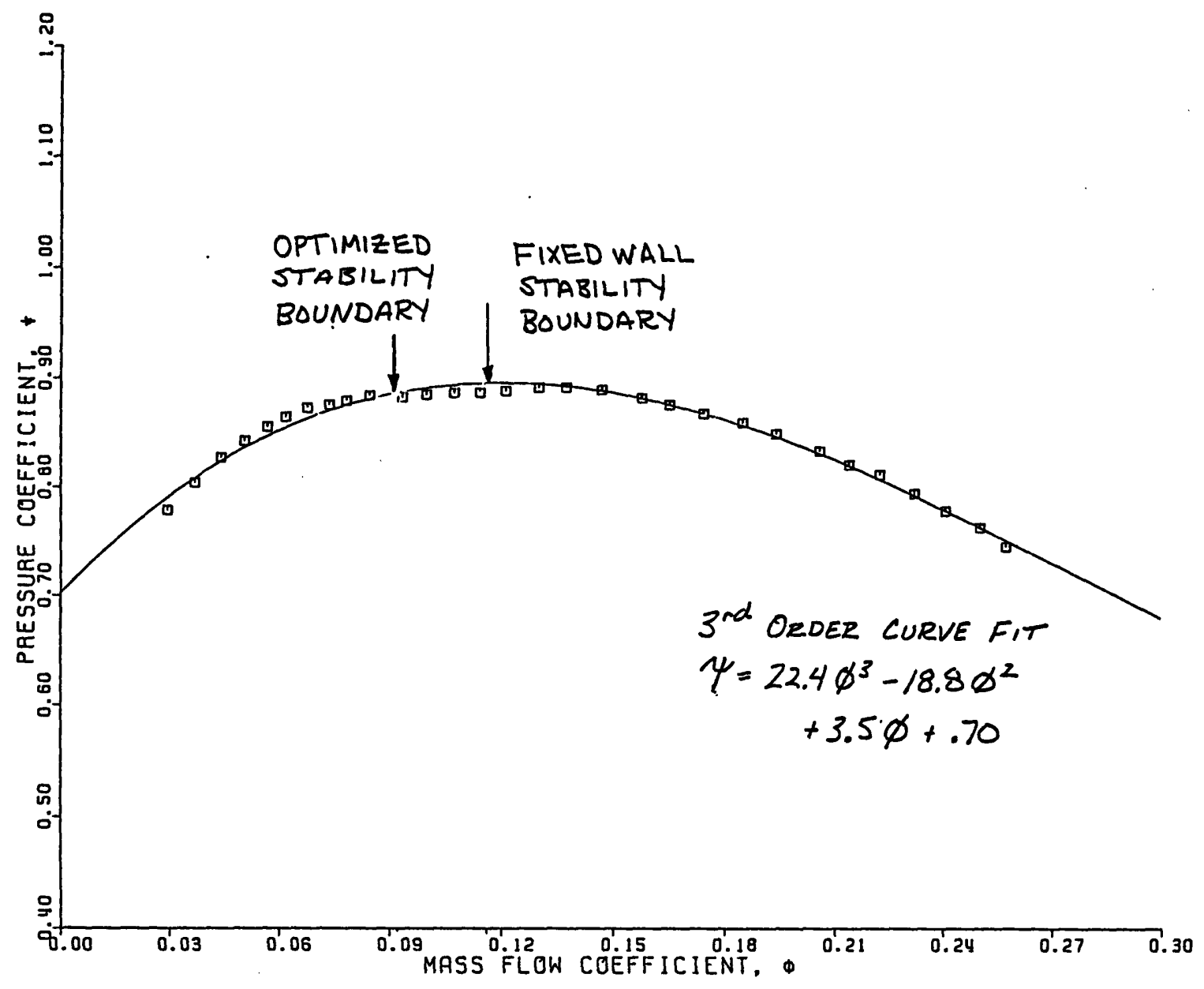

Figure 2.1: $70 \mathrm{~K}$ rpm Speedline Recorded with Close-Couple Throttle (from Pinsely 1988) 


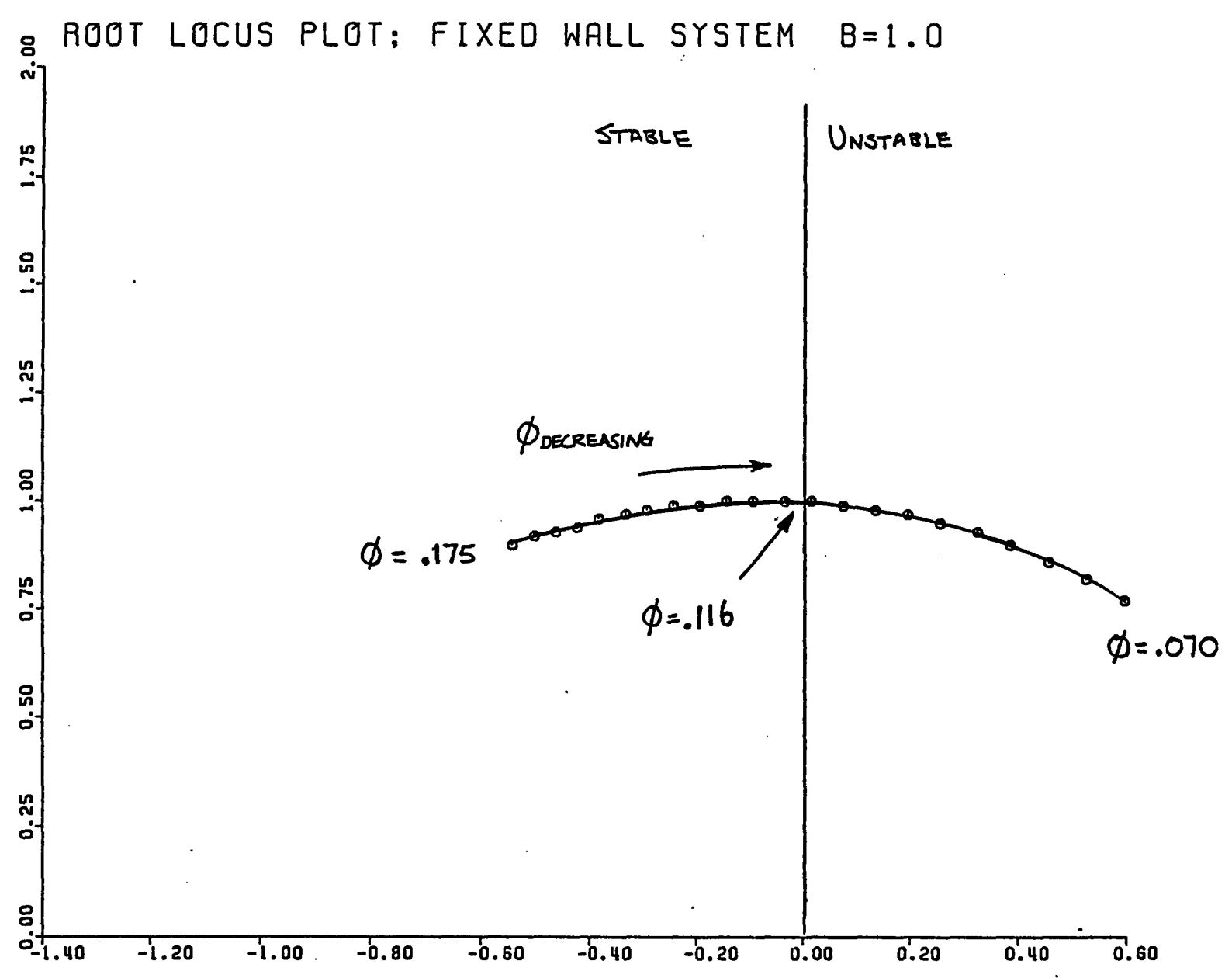

Figure 2.2: Root Locus Plot of Fixed Wall System $(B=1.0)$ 


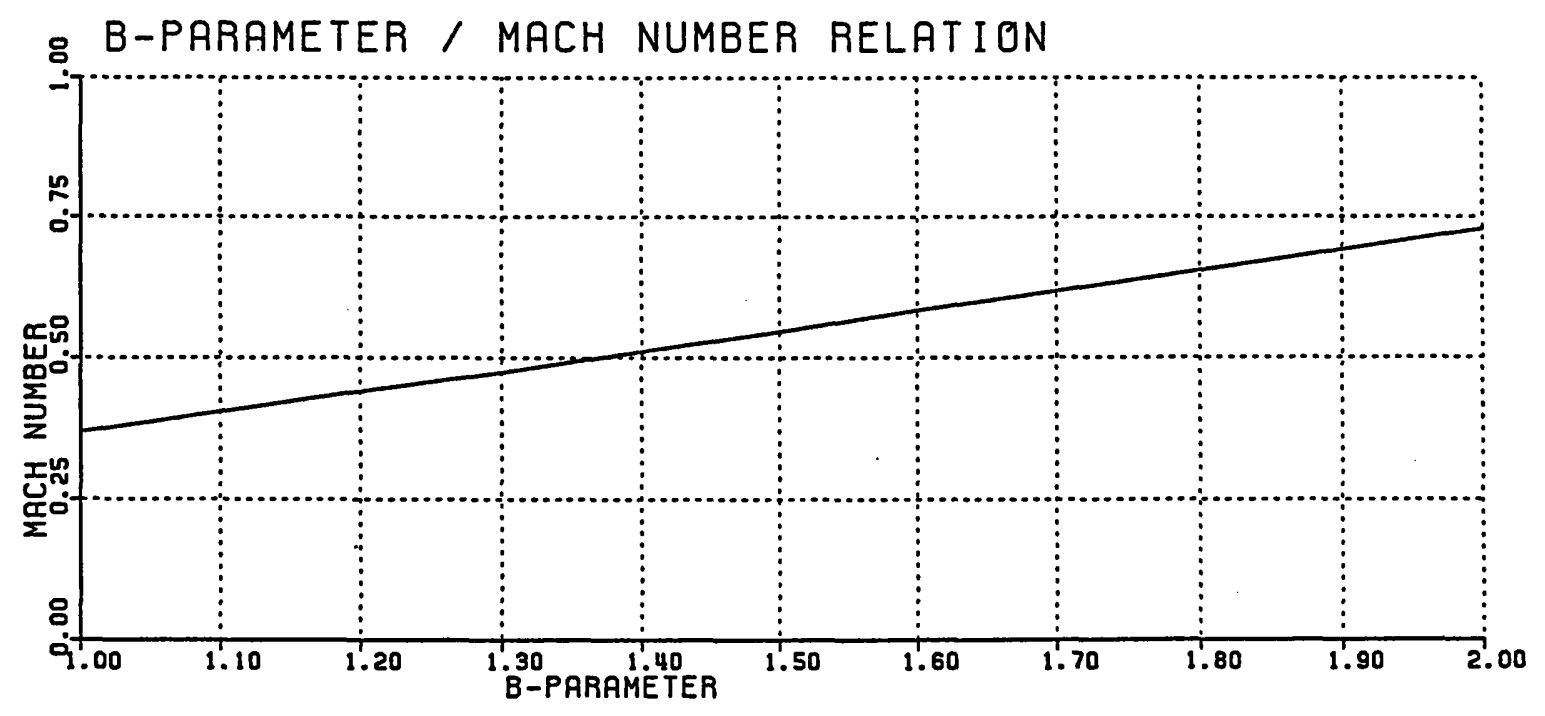

Figure 2.3: B-parameter / Tip Mach Number Relation 


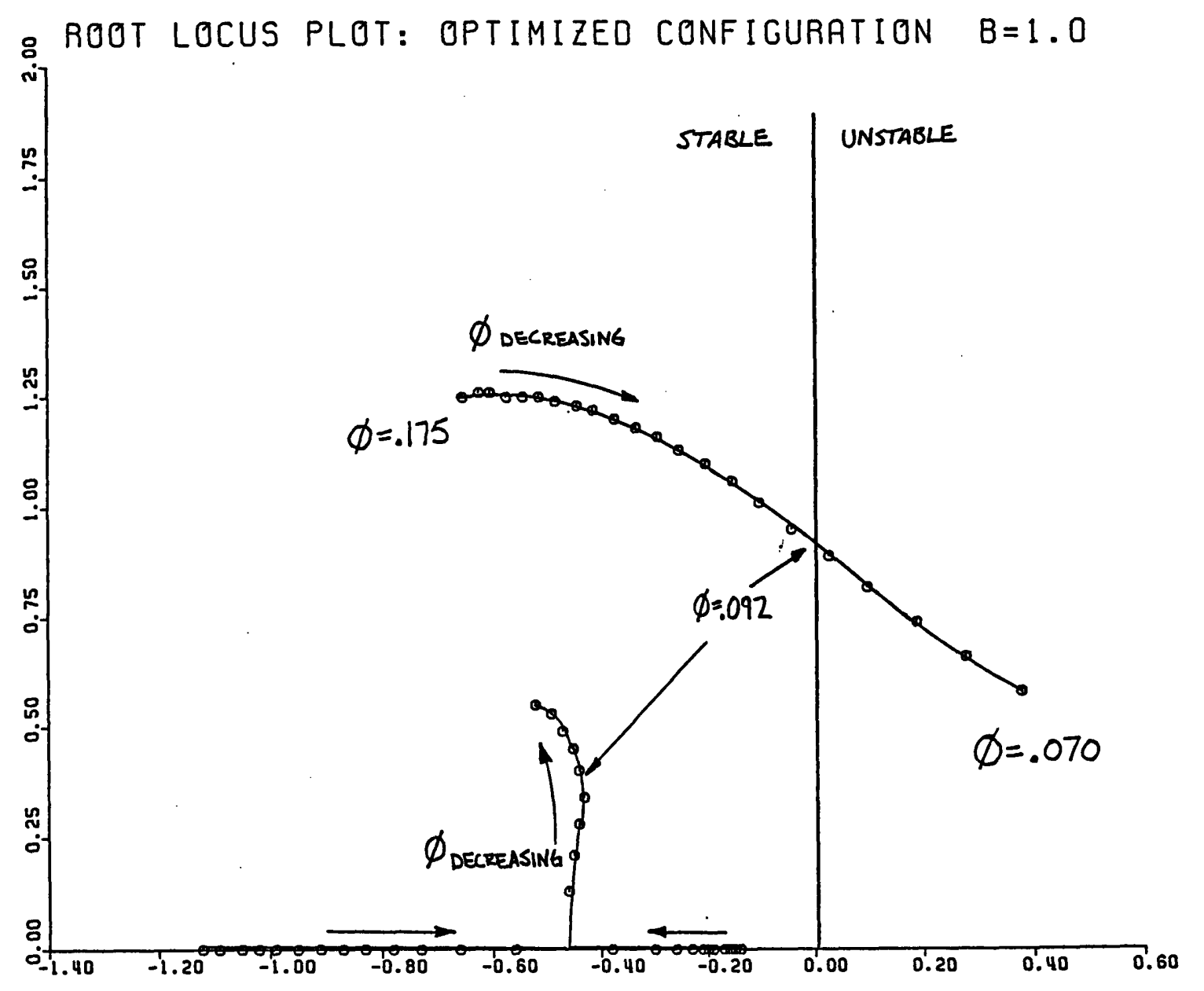

Figure 2.4: Root Locus of Optimized system $(B=1.0)$ 


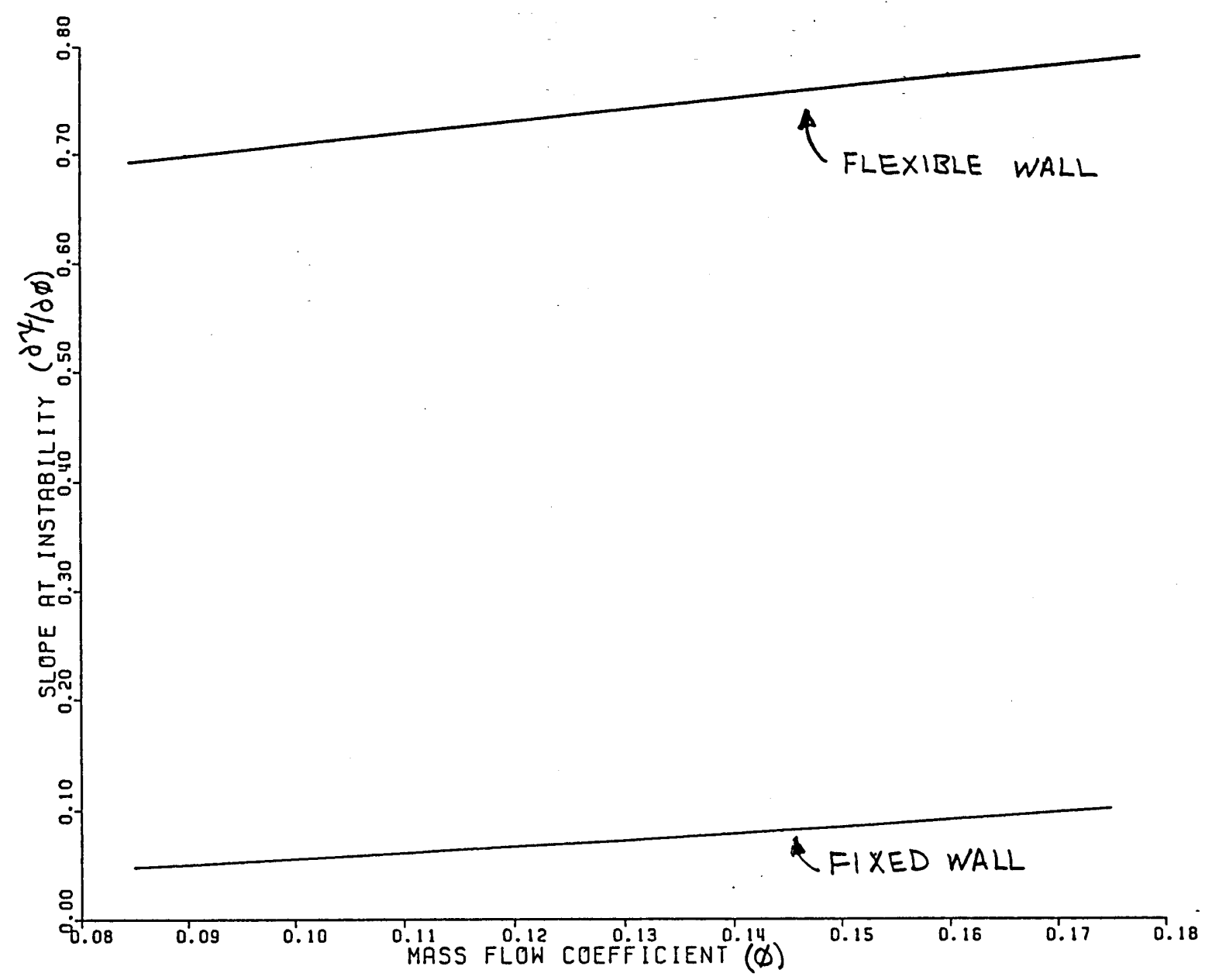

Figure 2.5: Effect of Mass Flow Coefficient on Maximum

Stable Compressor Slope for Fixed Wall and Optimized Systems $(B=1.0)$ 


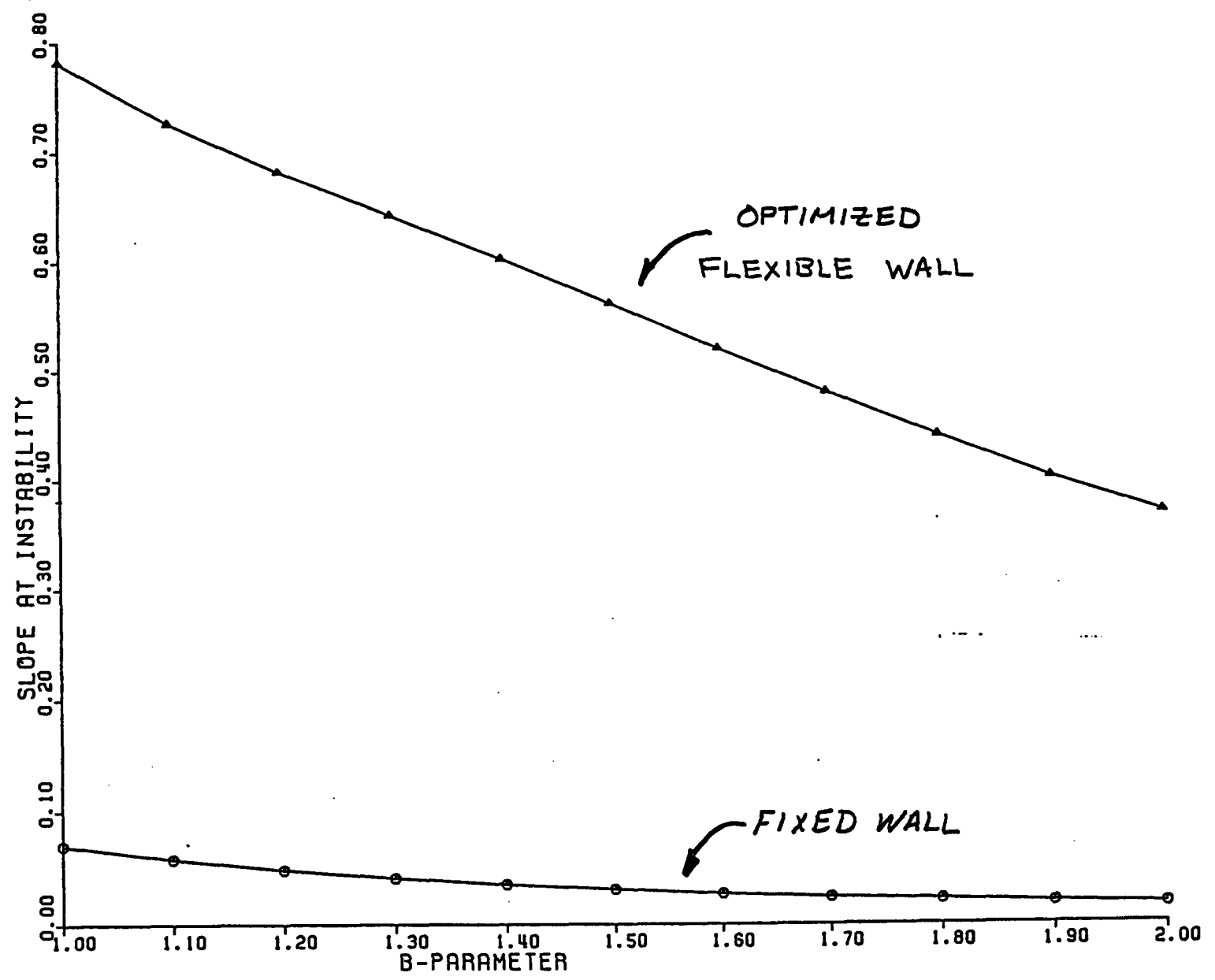

Figure 2.6: Maximum Controllable Slope versus B-Parameter (Fixed Wall and Optimized Systems )

$$
(B=1.0)
$$



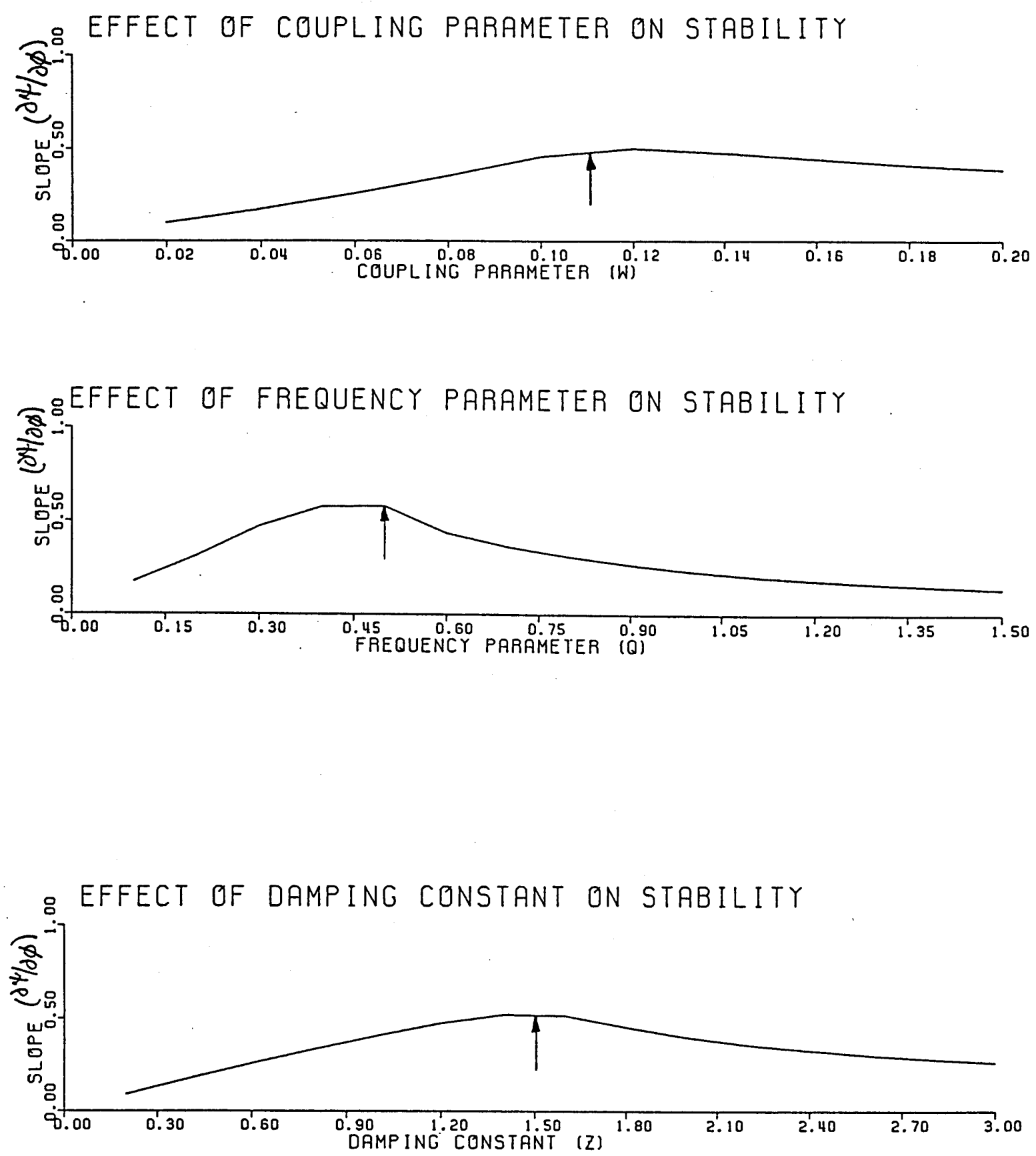

Figure 2.7: Maximum Controllable Slope for the Optimized System (structural control parameters varied independently) ( $B=1.5)$ 

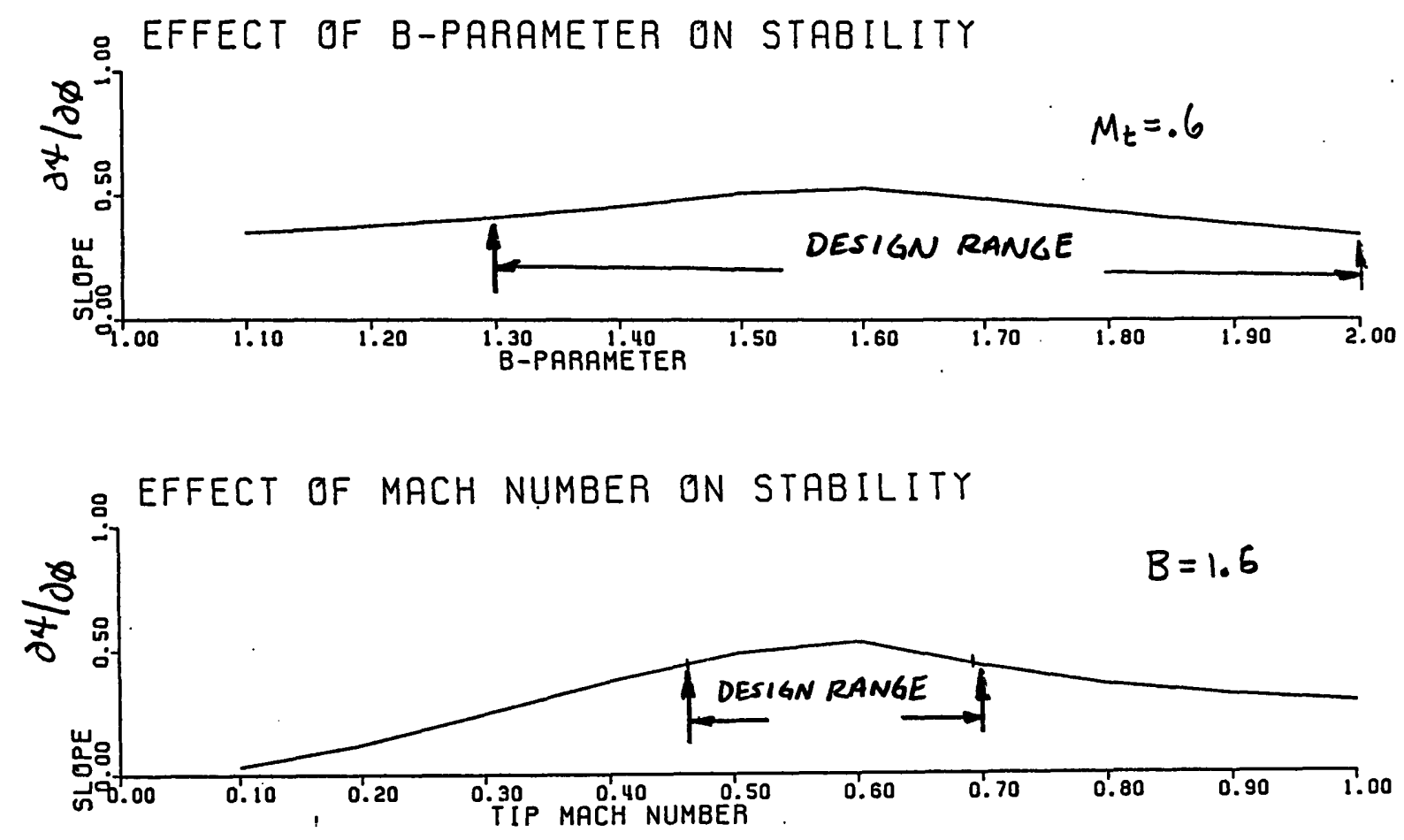

Figure 2.8A: Maximum Controllable Slope for Optimized System (Mach number and B-parameter varied independently)

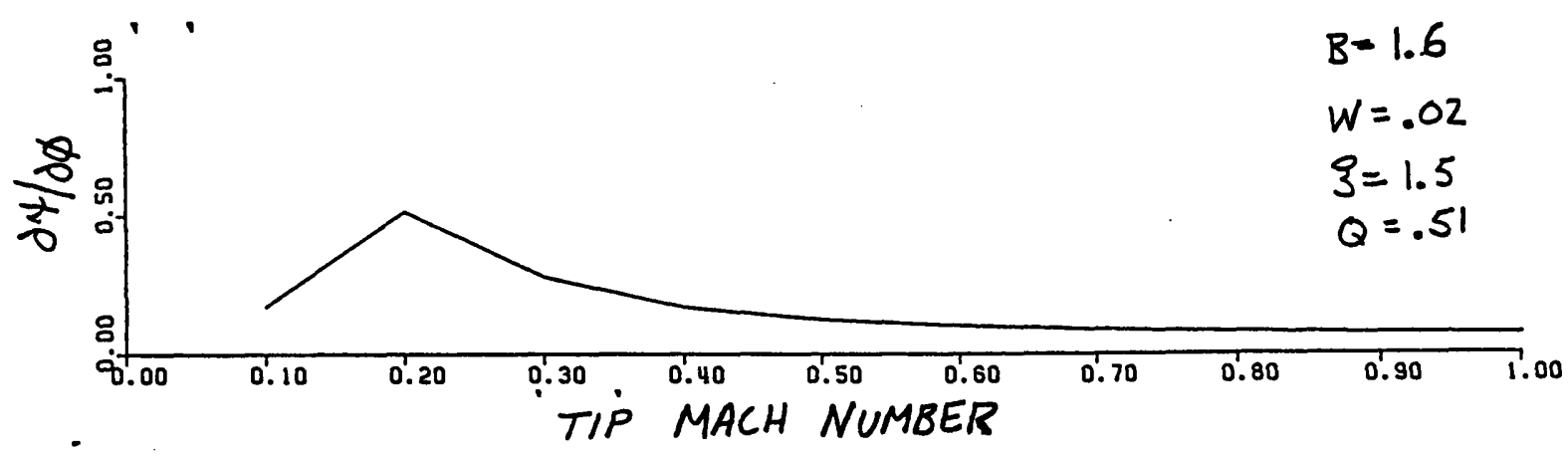

Figure 2.8B: Maximum Controllable Slope for System Optimized For $\mathrm{M}_{\mathrm{t}}=0.2$ 


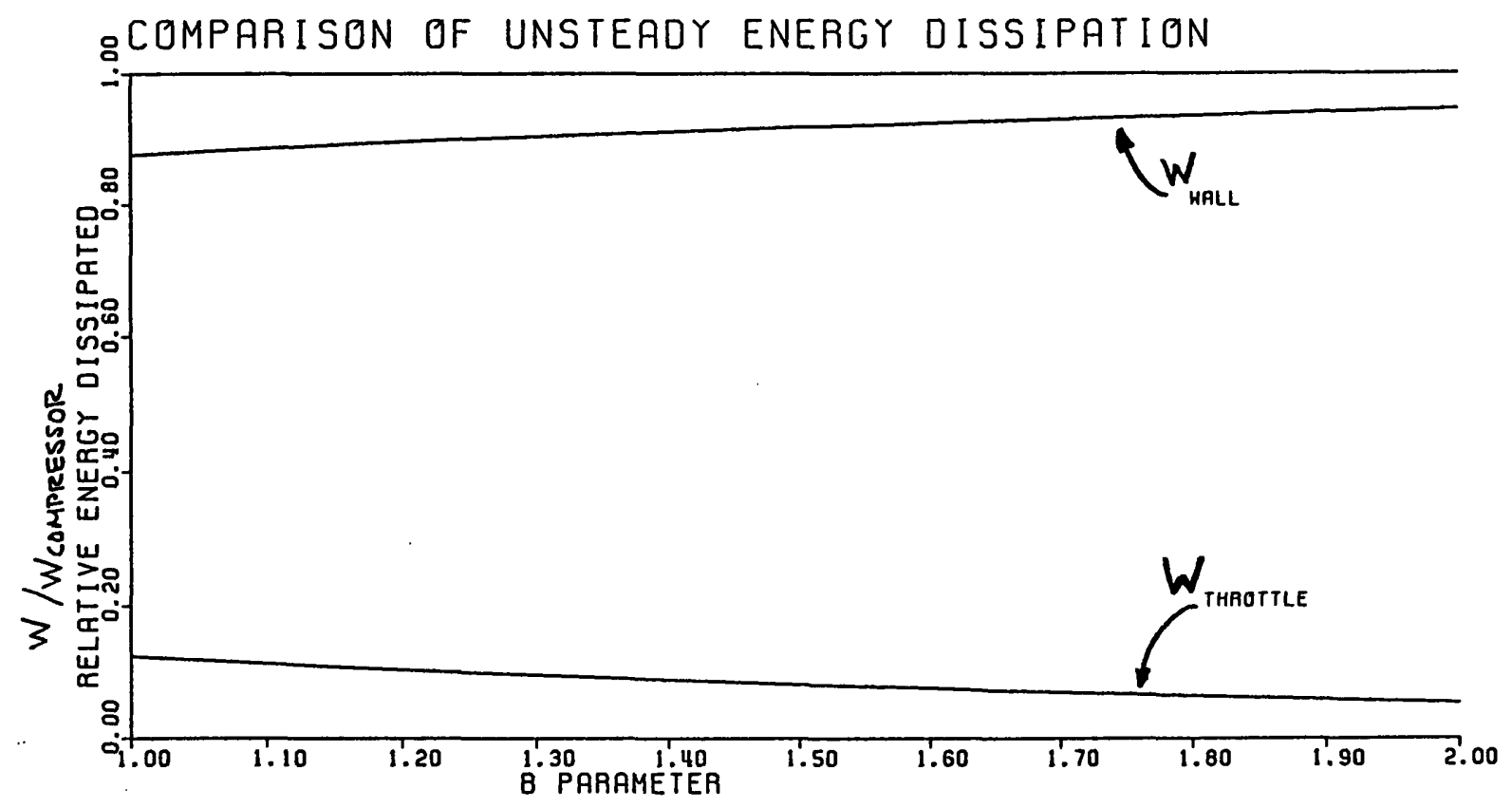

Figure 2.9: Comparison of Unsteady Energy Dissipation at the Neutral Stability Point 

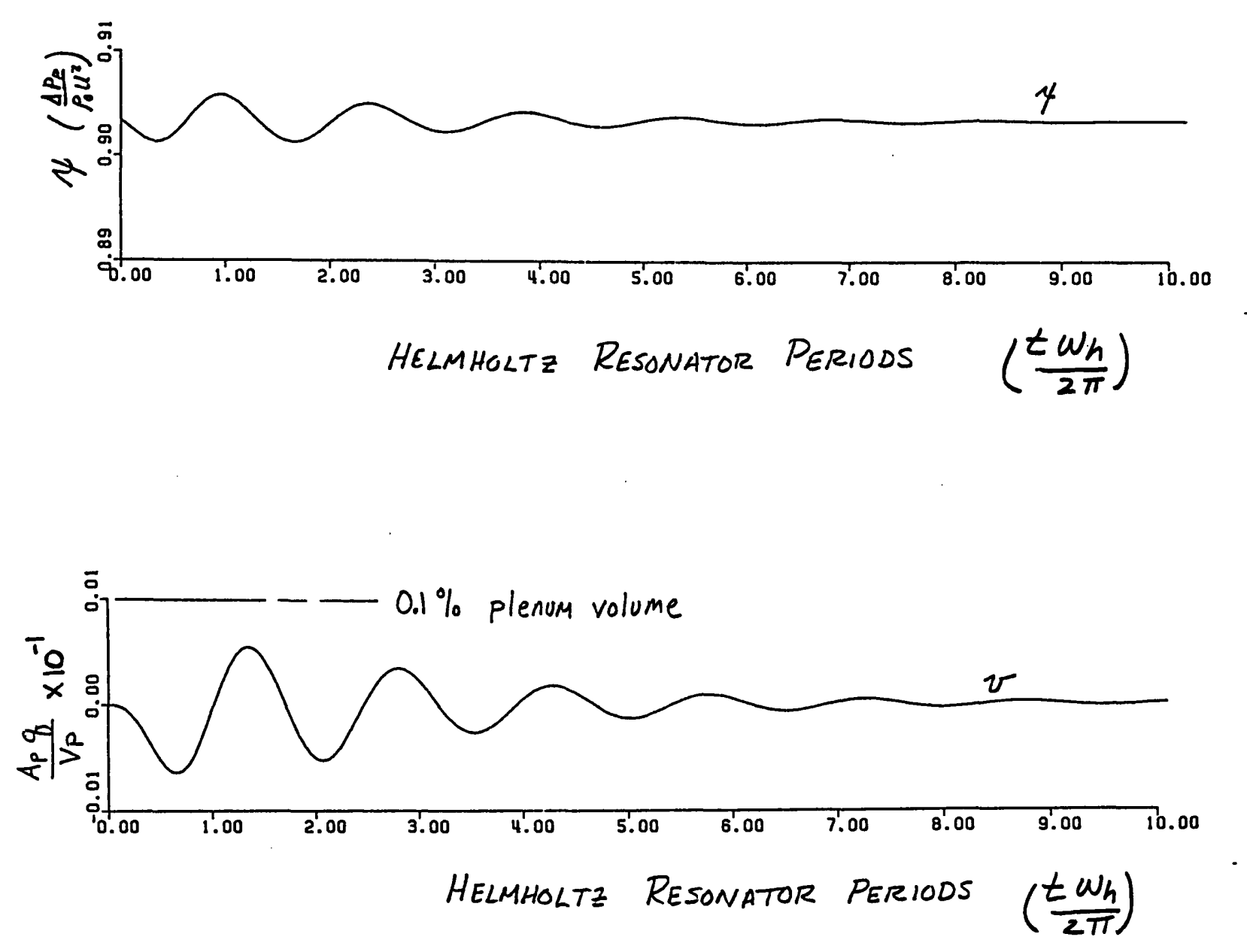

Figure 2.10: Time History Response to a 20\% Ambient Pressure

Disturbance $(B=1.5)$ 


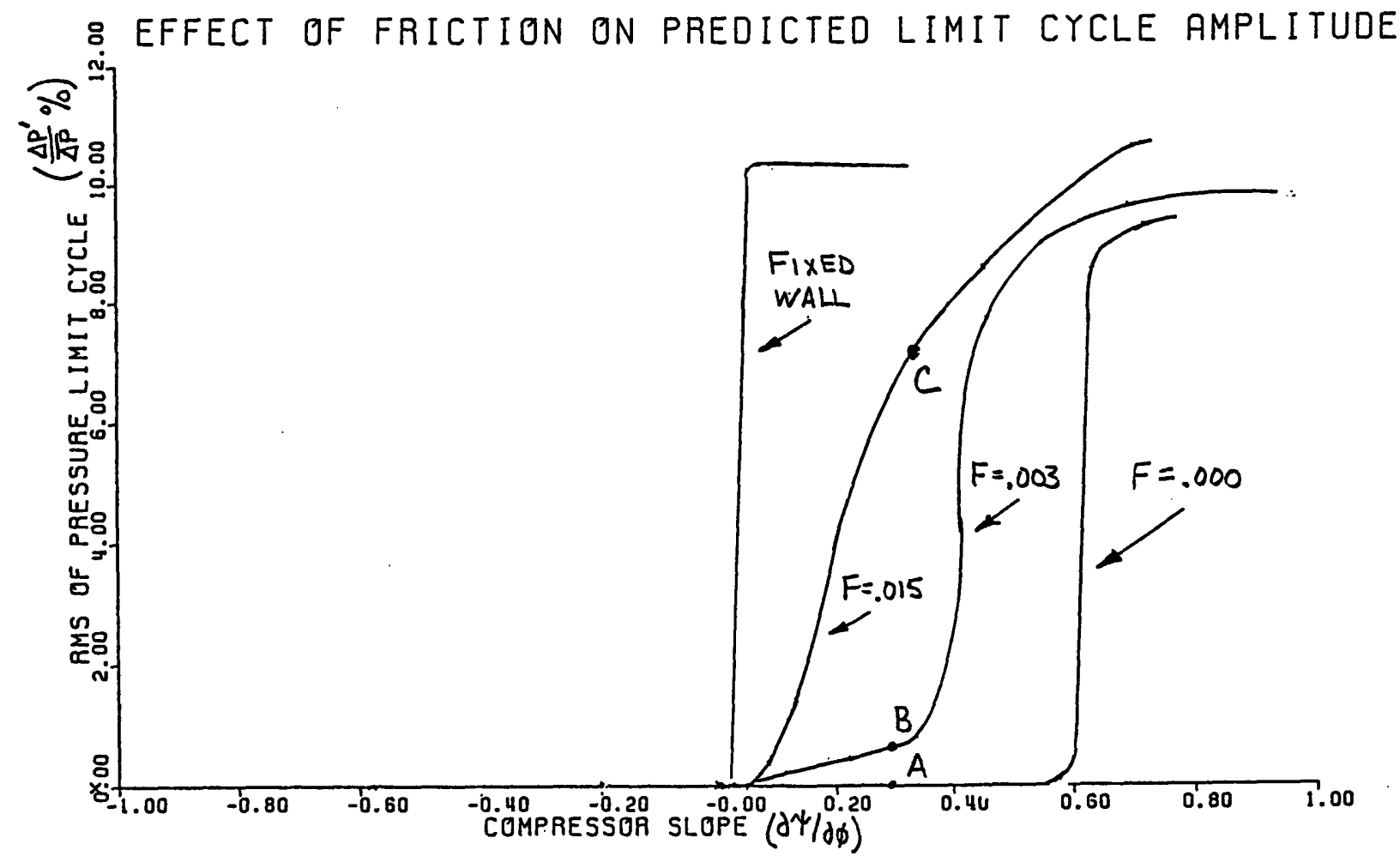

Figure 2.11: Predicted Limit Cycle Amplitude Versus Local Compressor Slope for Various Friction Levels 

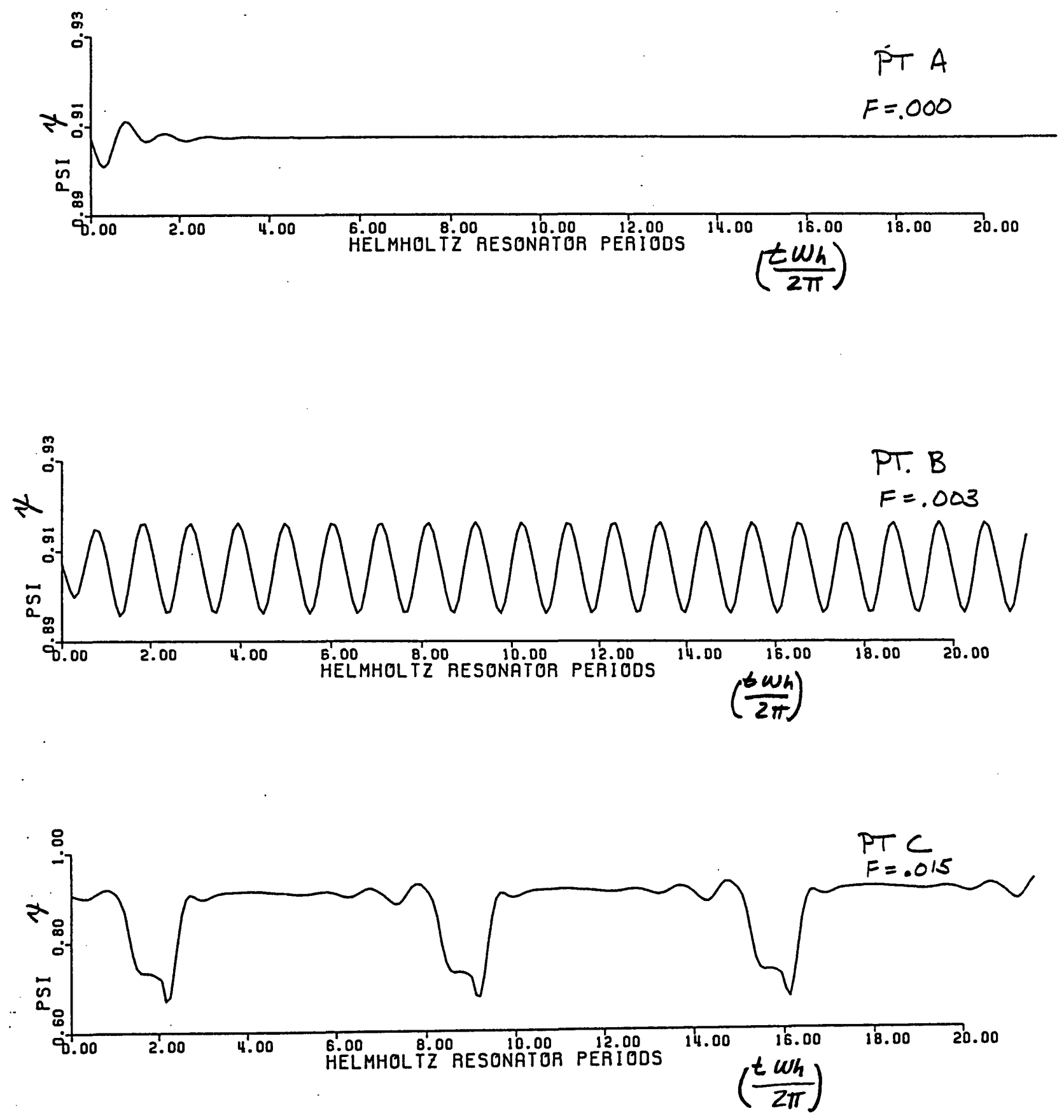

Figure 2.12: Predicted Limit Cycles for Various Friction Levels 


\section{DESIGN OF THE EXPERIMENTAL FACILITY}

\subsection{INTRODUCTION}

The analyses predict that a properly tuned, flexible plenum wall could substantially increase the stable flow range of typical compression systems. To investigate this experimentally and thus demonstrate passive surge control, a design study was undertaken to match the non-dimensional control parameters, while minimizing the effects of non-linearities, in a physically realistic device.

\subsection{EXISTING FACILITIES}

The conceptual design of the rig was based on an existing centrifugal compressor facility, constructed to investigate active throttle control of surge. The facility is described in

detail by Pinsley ${ }^{9}$, however, for completeness, the major components will be outlined in this thesis.

The centrifugal compressor was a Holset model H1D turbocharger developed for diesel engines. The impeller has an inlet area of $.00125 \mathrm{~m}^{2}$ with a hub to tip radius ratio of .37 . The impeller exit tip diameter is $.055 \mathrm{~m}$. The compressor has no inlet guide vanes, 6 blades, 6 splitter blades, and a vaneless diffusor. A performance map supplied by Holset is shown in Figure 3.1. The compressor is driven by a radial inflow turbine supplied by the high pressure lab air supply at the inlet. The turbine exhaust was connected to low pressure suction pulled by the laboratory steam ejector.

A schematic of the basic compression system facility is shown in Figure 3.2. During operation, air is drawn into the turbocharger through a bellmouth inlet, pressurized across the compressor and discharged into a plenum. From the plenum, the air exhausts across a throttling valve into a settling chamber. The air then exhausts to the atmosphere across a low pressure drop orfice plate. The settling chamber was designed to be dynamically isolated from the surge dynamics, also described by Pinsley 9 . 
The design of the compression system allowed for modification of the inlet duct length, plenum volume, and for the capacity to operate over a wide range of conditions. There was thus a large amount of freedom in selecting the system dynamic characteristics.

\subsection{DESIGN OF FLEXIBLE PLENUM WALL}

\section{a. Aerodynamic Spring}

With the basic compression system already existing, most of the design challenges were in designing a flexible plenum wall that could respond to pressure fluctuations in a linear manner as the model required. The basic analysis only required that the plenum volume respond to pressure perturbations, with the dynamics as dictated by the non-dimensional control parameters. In the conceptual design phase, several different ways to mechanically implement this control scheme were reviewed. A major constraint was that the wall had to be capable of withstanding large steady state and transient pressure loading, yet still respond to small amplitude perturbations in plenum pressure. A rigid plenum wall and an aerodynamic spring were determined to be practical solutions to these constraints.

To serve as the flexible wall, the rigid piston was mounted on a shaft, guided by linear bearings, and allowed to float between the main plenum and an auxilliary plenum. The seal between the two plenums was made with a low friction, convoluted diaphragm. Although the two plenums were effectively isolated for high frequency pressure disturbances (i.e. surge oscillations), the steady state pressures were equalized by a small diameter tube, so that there was no steady state load on the piston. The compressiblity of the air in the auxilliary plenum could thus serve as a self-adjusting spring and the mass of the piston itself could serve as the mass.

A small mechanical spring was necessary to maintain a constant equilibrium position for the piston over various operating conditions since, in the steady state limit the aerodyamic spring had no preferred equilibrium postion. The mechanical spring also allowed the steady state position of the wall to be adjustable. 
Once the conceptual design was developed, sizing of the components and selecting the operating range of the compressor was done by matching the non-dimensional control parameters. These dictate the characteristics of components relative to other components, but place no limitations on the absolute size or operating point of any component. Figure 3.3, illustrates this by showing the mass of the piston versus area of the piston for a given auxiliary plenum volume required to match the non-dimensional control parameters. The plot also shows how the required auxillary plenum volume varies as a function of wall mass for various mechanical spring constants.

Since the presence of the steady state equalization tube could affect the behavior of the aerodynamic spring, the non-linear code was modified to quantify the effect of leakage between the two plenums. The leakage was modelled as the flow through an orifice plate -i.e. a parabolic pressure drop versus mass flow relation was used. The results of the analysis for B $=1.5$ are shown in Figure 3.4 , where the decrease in maximum slope before surge is plotted versus a non-dimensional orifice area for the optimized system at typical operating conditions. Leakage caused small amplitude limit cycles similar to those predicted to occur as a result of Coulomb friction. An example of a limit cycle resulting from the steady state pressure equalization leakage is shown in Figure 3.5. The calculated response is for the optimized configuration with $B=1.5$ and $\phi=.095$ on the $70 \mathrm{~K}$ speedline. The leakage is modelled as flow through a $.003 \mathrm{~m}$ diameter orifice plate. Without the leakage term modelled, a small disturbance decays; with the leakage, the small disturbance grows into a limit cycle as shown. As shown, leakage should be minimized for this design to successfully suppress surge.

\section{b. Viscous Damper}

It was determined in the conceptual design stage that a viscous dashpot could provide the damping required if the effects of Coulomb friction were small. It was also determined that the damper should be mounted co-linearly with the piston shaft to minimize side load on the linear bearing guiding the shaft. 
In the initial stages of sizing the components of the flexible wall, it was found that commercially available dashpots quoted unacceptable friction levels. To avoid this problem, a low friction, pnuematic, double acting actuator was modified to serve as a damper. The actuator was filled with 5 w30 oil and the ports on either end were connected through a variable area valve. In this configuration, oil was forced through the valve when the actuator was displaced, yielding a quasi-linear, adjustable dashpot. Testing of various dashpots developed from the same basic design, showed that the dashpot was closely linear over the expected range of wall velocities, indicating that viscous dissipation was the dominant damping mechanism within the dashpot.

Displacement limiters were installed to limit the maximum wall displacement to $+/-.5$ inches (approximently $5 \%$ of plenum volume) during surge, much greater than the predicted range required to suppress ambient disturbances.

The finalized rig specifications are shown below and a detailed drawing of the flexible plenum wall apparatus is shown in Figure 3.6. 
Design Specifications for the Flexible Plenum Wall

Experimental Facility

$\begin{array}{ll}\text { B Parameter } & 1.3 \text { to } 2.0 \\ \text { Wheel Speed } & 60,000 \mathrm{rpm} \text { to } 100,000 \mathrm{rpm} \\ \text { Tip Speed } & 170 \mathrm{~m} / \mathrm{s} \text { to } 290 \mathrm{~m} / \mathrm{s} \\ \text { Helmholtz Frequency } & 18.5 \mathrm{~Hz} \text { to } 19.9 \mathrm{~Hz} \\ \text { W Parameter } & 0.11 \\ \text { Q Parameter } & 0.51 \\ \zeta \text { Parameter } & 1.5 \text { to } 3.0 \\ \text { Area of Wall } & .0669 \mathrm{~m}^{2} \\ \text { Mass of Wall } & 6.2 \mathrm{~kg} \\ \text { Volume of Plenum } & 0.0108 \mathrm{~m}^{3} \\ \text { Inlet Duct Length } & 1.16 \mathrm{~m} \\ \text { Inlet Area } & 0.00125 \mathrm{~m}^{2} \\ \text { Auxiliary Plenum Volume } & 0.0388 \mathrm{~m} 3 \\ \text { Plenum Operating Temperature } & 340 \mathrm{~K} \mathrm{to} 390 \mathrm{~K} \\ \text { Pressure Rise } & 30 \mathrm{KPa} \text { to } 90 \mathrm{KPa} \\ \text { Plenum Operating Density } & 1.4 \mathrm{~kg} / \mathrm{m}^{3} \text { to } 1.7 \mathrm{~kg} / \mathrm{m}^{3} \\ \text { Aerodynamic Spring Constant } & 24,000 \mathrm{n} / \mathrm{m} \mathrm{to} 35000 \mathrm{n} / \mathrm{m} \\ \text { Mechanical Spring Constant } & 2100 \mathrm{n} / \mathrm{m} \\ \text { Damping Ratio } & 1000 \mathrm{n} \mathrm{s} / \mathrm{m} \mathrm{to} 2000 \mathrm{n} \mathrm{s} / \mathrm{m} \\ \text { Maximum Wall Motion } & +/ 1.25 \mathrm{~cm} \\ \text { Coulomb Friction } & 10 \mathrm{n}\end{array}$

\subsection{RIG INSTRUMENTATION}

The rig was instrumented for both steady state and time resolved measurements. The following quantities were recorded:

1) inlet mass flow,temperature and pressure

2) main and auxillary plenum pressure/temperature

3) system exit mass flow

4) wall displacement

5) force on damper

6) wheel speed 
(The transient quantities recorded are listed in Section 3.5)

Inlet mass flow measurements were recorded using a TSI hot wire anemometer. The hot wire was placed approximately 1-diameter downstream of the bellmouthed inlet. The boundary layer displacement thickness was estimated by assuming laminar flow over a flat plate $^{14}$ given as:

$$
\delta=\frac{1.72 \mathrm{x}}{\sqrt{\mathrm{Re}_{\mathrm{x}}}}
$$

For typical flow conditions, blockage due to the boundary layer displacement thickness was calculated to be less than $1 \%$ of the of the flow area and was therefore neglected. The hot wire was used to measure fluctuations in mass flow about the mean only, due to difficulties associated with DC drift. To measure these fluctuations in mass flow, the slope of the transducer output voltage versus mass flow rate curve was needed. The voltage versus mass flow calibration curve, as measured by the steady state orifice plate, is shown in Figure 3.7 . Hot wire anemometer output was filtered with a $200 \mathrm{~Hz}$ low pass filter contained within the TSI anemometer.

The steady state inlet, main plenum, auxilliary plenum, and upstream of the orifice temperatures were measured using an Omega type $\mathrm{K}$ thermocouple.The error of the thermocouple is estimated at $+/-1.6 \mathrm{~F}$. The compressor bearing temperature was also monitored to prevent the bearing from overheating. The oil recirculation system used to lubricate the bearings is desribed by Pinsley 9 .

The turbocharger rotational speed was measured using a once per revolution magnetic pickup. The pulses were fed into an Analog Devices frequency to voltage converter, which was calibrated against an HP 5310A Universal counter. The calibration is shown in Figure 3.8.

The main plenum static pressure was recorded using a Druck model PDCR 820, 0-30 psi pressure transducer. The frequency response of a similar pressure measurement system using the same tranducers, amplifiers and mounting techniques was examined by Pinsley 9 
who found no appreciable lag or attenuation of the signal for the range of relevant frequencies $(8-15 \mathrm{~Hz})$. The transducer was supplied with 10 volts excitation and the output voltage was amplified to approximately 10 volts full scale with an Analog Devices 2B31L high performance strain gauge signal conditioner. The cutoff frequency was set at $100 \mathrm{~Hz}$.

Calibration of the pressure transducer was performed against a Wallace-Tiernan (Model FA 145) 0-35 psi gauge pressure with an estimated precision of $+/-0.01 \mathrm{psi}$. The transducer calibration was routinely checked against the Wallace-Tiernan gauge. A typical calibration is shown in Figure 3.9 .

The position of the wall was measured using a Trans-Tek model 244-0000 DCDC displacement transducer, supplied with $+/-15$ volts excitation. The manufacturer's quoted frequency response range of $0-100 \mathrm{~Hz}$ with $0.5 \%$ linearity over the total working range of 4 inches was assumed to hold. A two point calibration of the transducer is shown in Figure 3.10 .

The force on the damper was measured to provide a means to calculate a damping constant for the damper and to investigate the actual damping characteristics of the quasiviscous dashpot. The force was measured with a strain gauge bridge attached to a section of steel with a contracted section in series with the damper and the pistion shaft as shown in Figure 3.6. The strain gauges were positioned on the contracted section to be insensitive to bending deflections yet amplify stretching and compression. The strain gauge bridge was exicted with 10 volts and the output signal was amplified to approximately $50 \mathrm{lbs}$ per volt using an Analog Devices 2B31L high performance strain gauge signal conditioner. The cutoff frequency was set at $50 \mathrm{~Hz}$. A force versus voltage calibration for the force transducer is shown in Figure 3.11 .

The auxillary plenum was instrumented with pressure and temperature instrumentation identical to the main plenum. The calibration curve for the auxillary plenum pressure transducer is shown in Figure 3.12 
The steady state mass flow was measured with an orfice plate located downstream of the throttle valve, at the exit of a settling chamber. The flow temperature and pressure were recorded upstream of the orifice. Pressure was recorded using a 2.5 psi Druck model PDCR 811 silicon strain gauge bridge transducer, excited with 10 volts and the output was amplified to approximately 10 volts full scale with the Analog Devices amplifier described above. Calibration of the pressure transducer was performed against a 0-15 psi Wallace-Tiernam (Model FA 145) with an estimated precision of 0.005 psi. pressure gauge.and is shown in Figure 3.13. The cutoff frequency was set at $100 \mathrm{~Hz}$.

The orifice plate was a standard, 1.75 inch diameter, ASME, sharp-edged orifice plate, and therefore, could be calibrated empirically 15 . The empirical calibration isolated the effects of compressibility and Reynolds number. The empirical calibrations, quoted to be accurate to approximately $0.2 \%$, were developed based on large amounts of data obtained using standard sharp edged orifice plates for the measurement of air flow. The mass flow through the orifice $^{15}$ is given by:

$$
\dot{\mathrm{m}}=\varepsilon\left(\frac{\Delta \mathrm{P}}{\mathrm{P}_{0}}\right) \alpha(R e) a_{p} \sqrt{\rho_{o r} \frac{\Delta \mathrm{P}}{1-\mathrm{m}^{2}}}
$$

where $m$ denotes the ratio of orifice area to the upstream flow area. The orifice measuring system in this facility had $m=.19 . \varepsilon$ is the compressibility correction term and is given empirically 15 as:

$$
\varepsilon=1-\beta \frac{\Delta \mathrm{P}}{\mathrm{P}_{0}}
$$

where $\beta$ is an empirical constant given as a function of $m^{15}$. For $m=.19, \beta$ is taken to be .32. $\alpha$ is the contraction ratio, given as a function of Reynolds number based on orifice diameter in Figure 3.14 . 


\subsection{DATA ACQUISTION}

The data acquistion was done using of an IBM AT personal computer equipped with an internal Data Translation model DT2801 analog to digital converter card. Analog signal ports were provided by a DT707 terminal panel, as described by Pinsley. Data acquisition routines were developed using Data Translations PCLAB software package.

The analog to digital system could read up to eight differential voltage channels. The following transient signals were sampled.

1) Inlet mass flow

2) Main plenum pressure

3) Auxillary plenum pressure

4) Force on the damper

5) Pressure across orifice

6) Wall position

7) Wheel speed

Steady state temperature measurements were recorded manually as displayed from an Omega thermocouple thermometer (Model 650).

The sampling rate of the A/D system was adjustable, but due to memory constraints, unsteady data records lengths were limited to 1000 samples per channel. For the 1 second transient data records, the sampling rate was set at $1000 \mathrm{~Hz}$, satisfying the Nyquist criteria for the time resolved measurements up to $500 \mathrm{~Hz}$. For the longer, 10 second record lengths, a sampling rate of $100 \mathrm{~Hz}$ was used. Although the lower sampling frequency violates the Nyquist criteria, comparison of the digital spectrum of signals sampled at $100 \mathrm{~Hz}$ to signals sampled at $1000 \mathrm{~Hz}$, showed that there was no significant frequency content above $50 \mathrm{~Hz}$ and therefore no significant aliasing was occuring. 


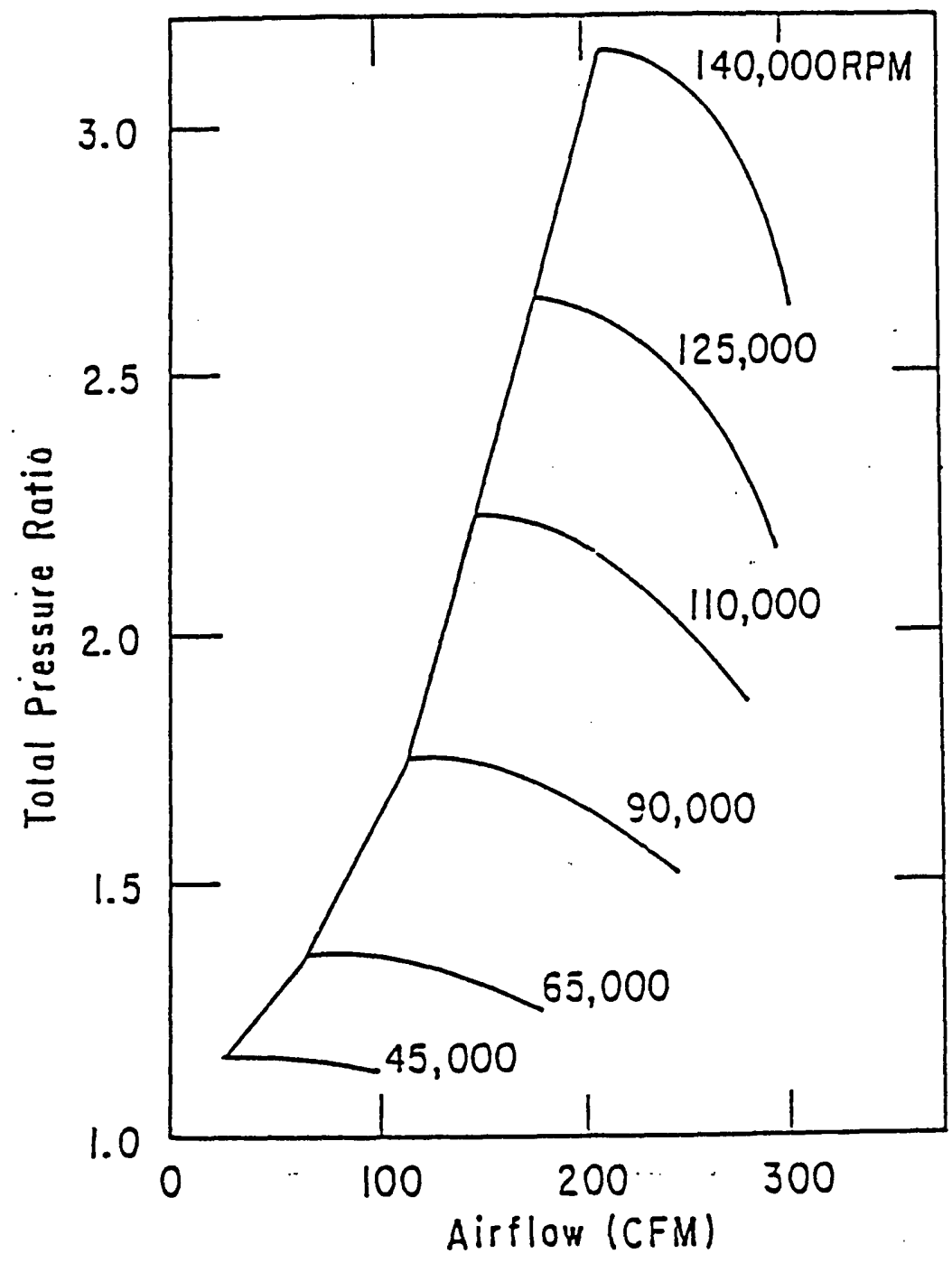

Figure 3.1: Compressor Performance Map for Holset H1D 


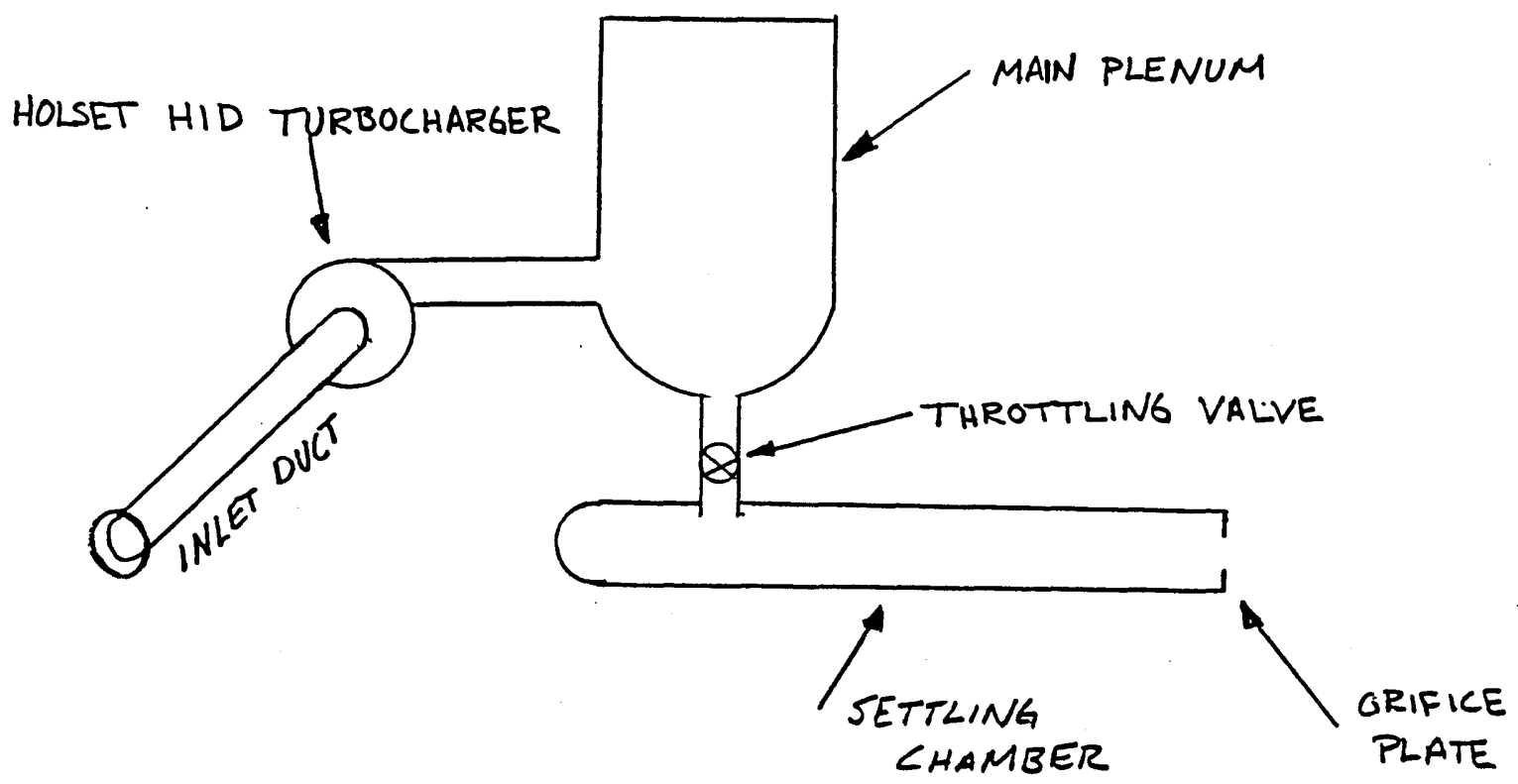

Figure 3.2: Schematic of Basic Compression System 


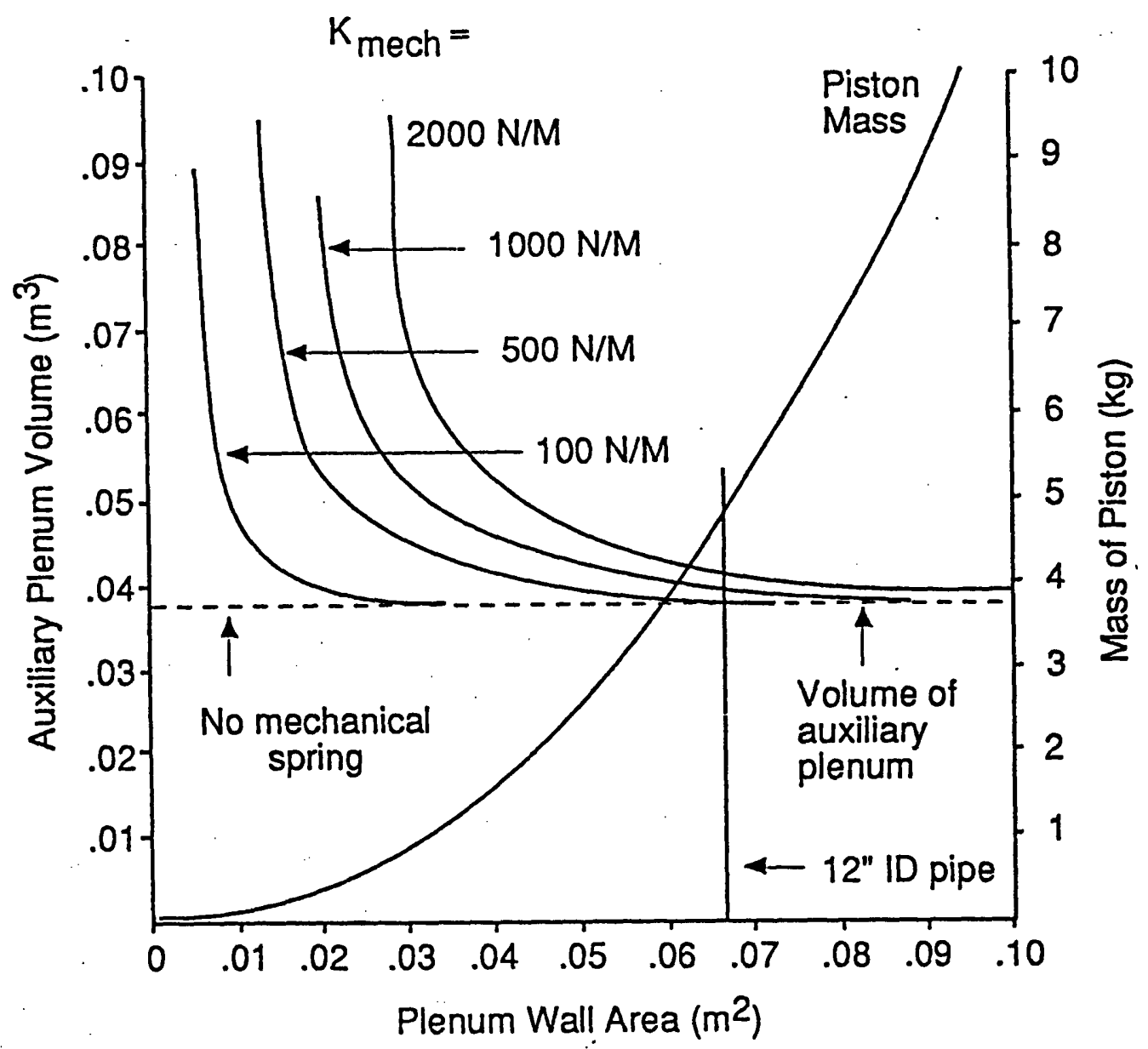

Figure 3.3: Preliminary Design Curves:Auxiliary Plenum Volume, Piston Mass And Area 


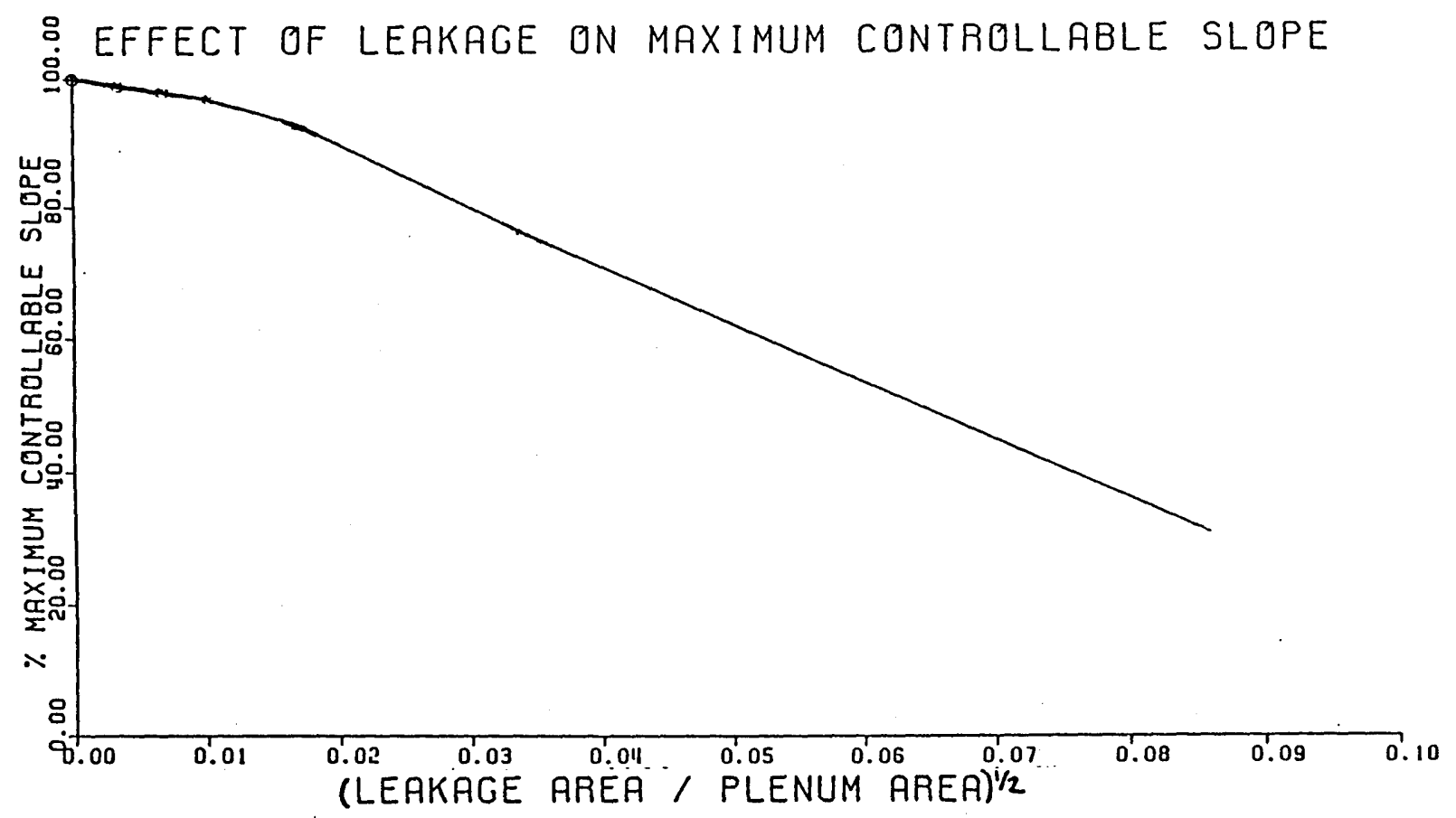

Figure 3.4: Effect of Leakage on Stability; Maximum Stable Compressor Slope with Leakage Divided by Maximum Slope without Leakage for Various Amounts of Leakage

$$
(\mathrm{B}=1.5)
$$




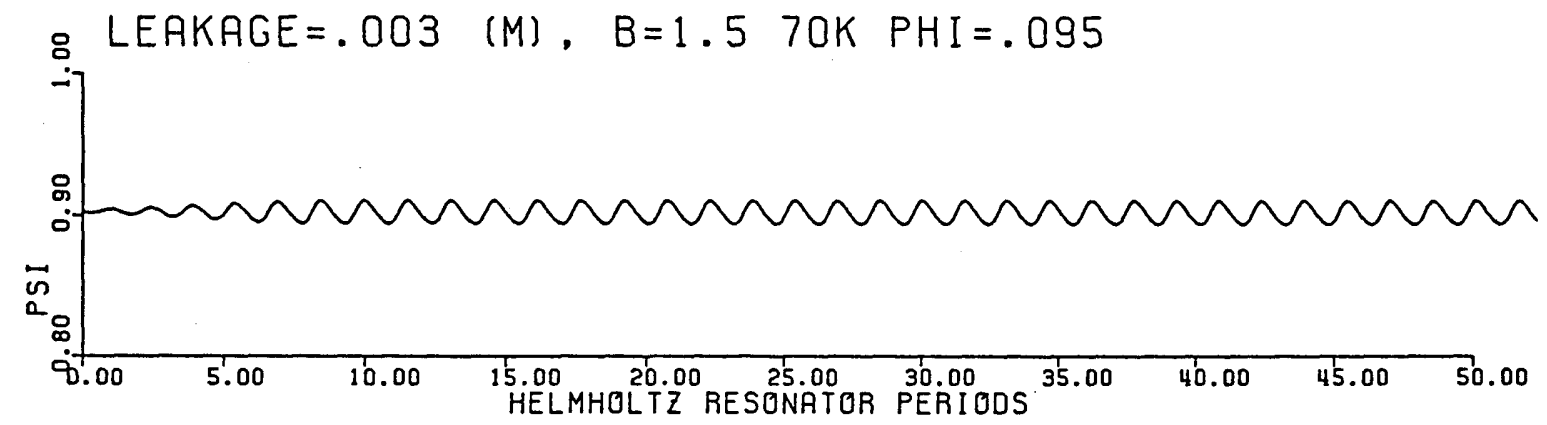

Figure 3.5: Limit Cycle Due to Leakage 


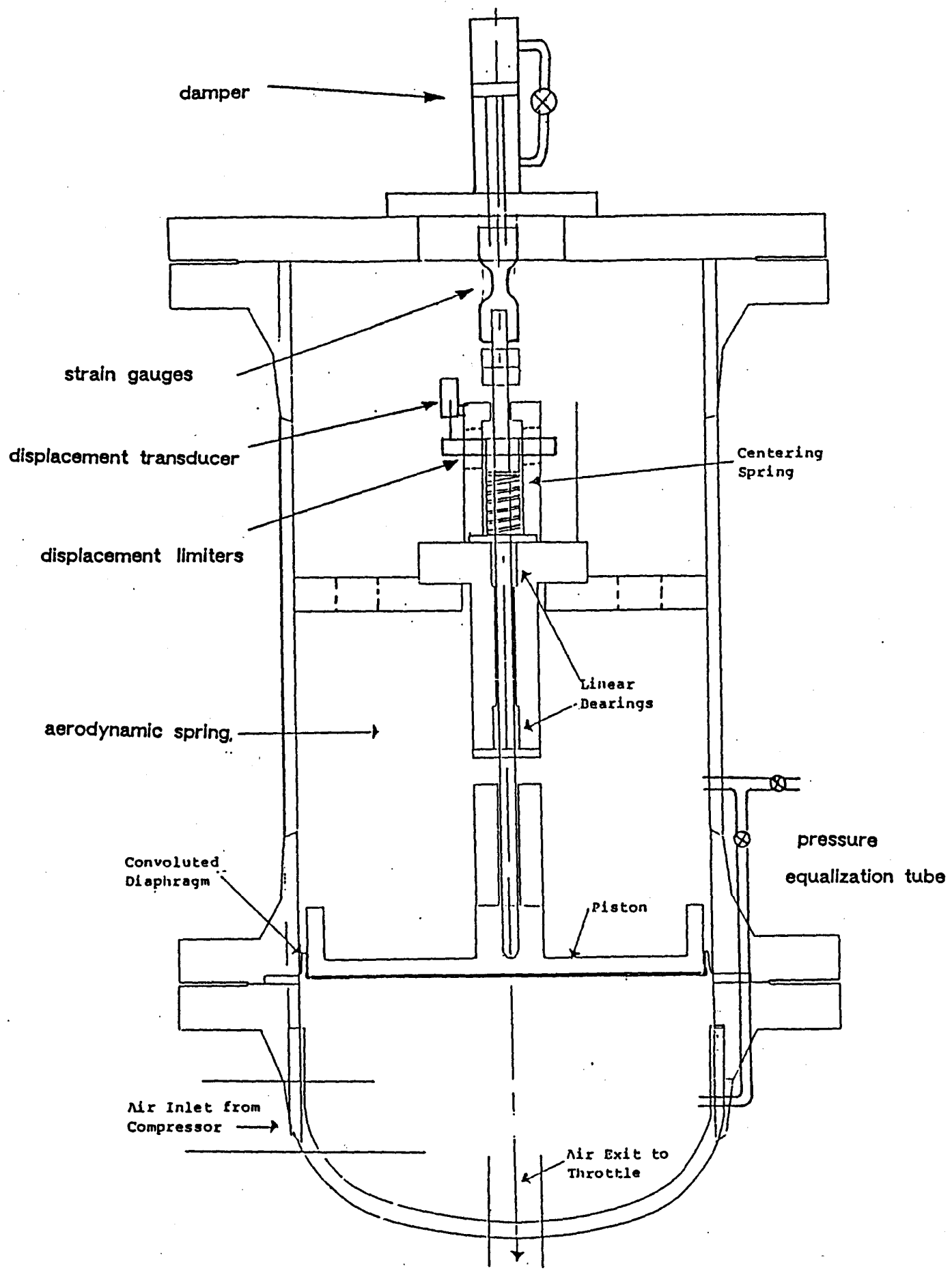

Figure 3.6: The Flexible Plenum Wall 


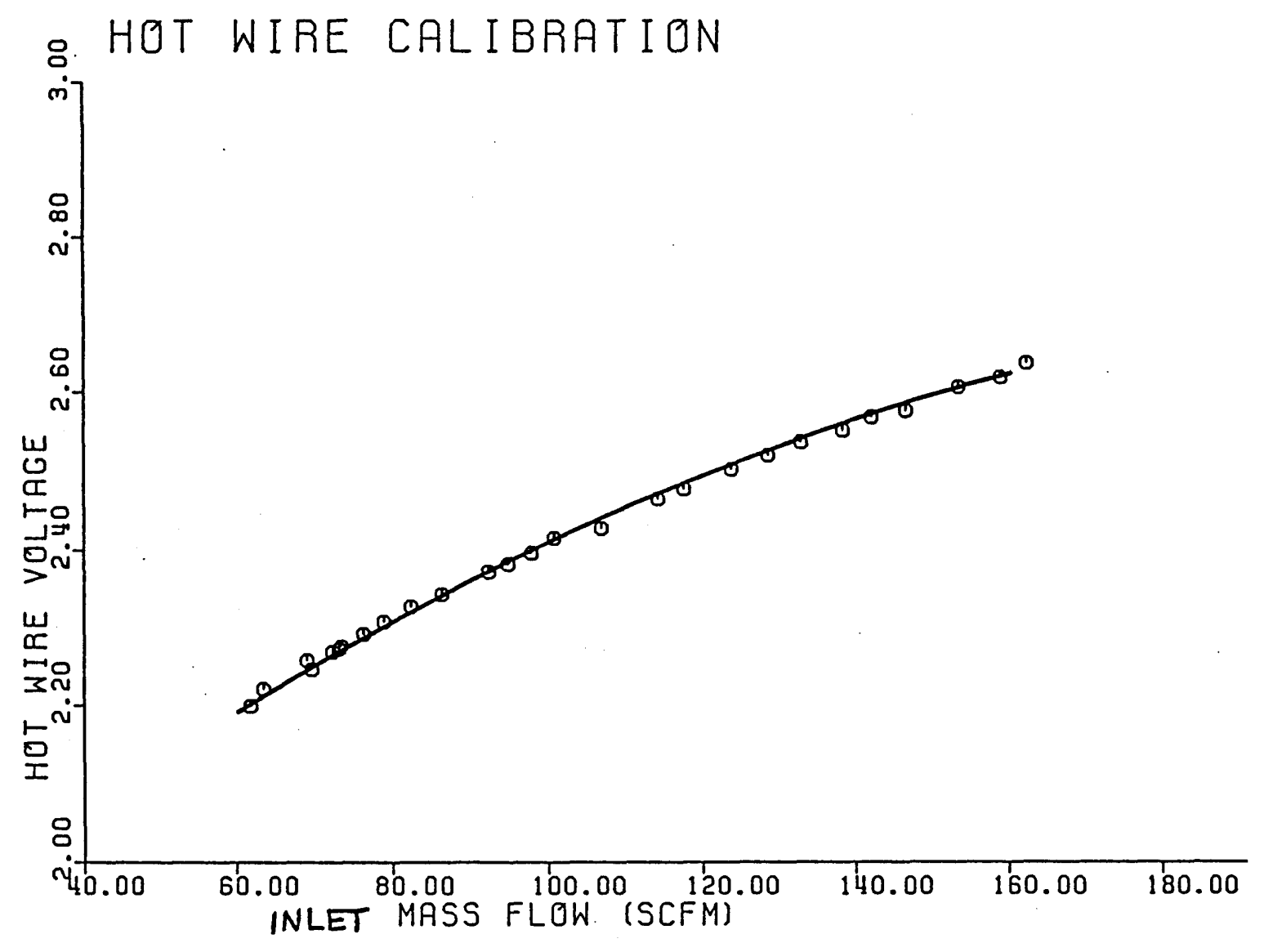

Figure 3.7: Inlet Hot Wire Calibration 


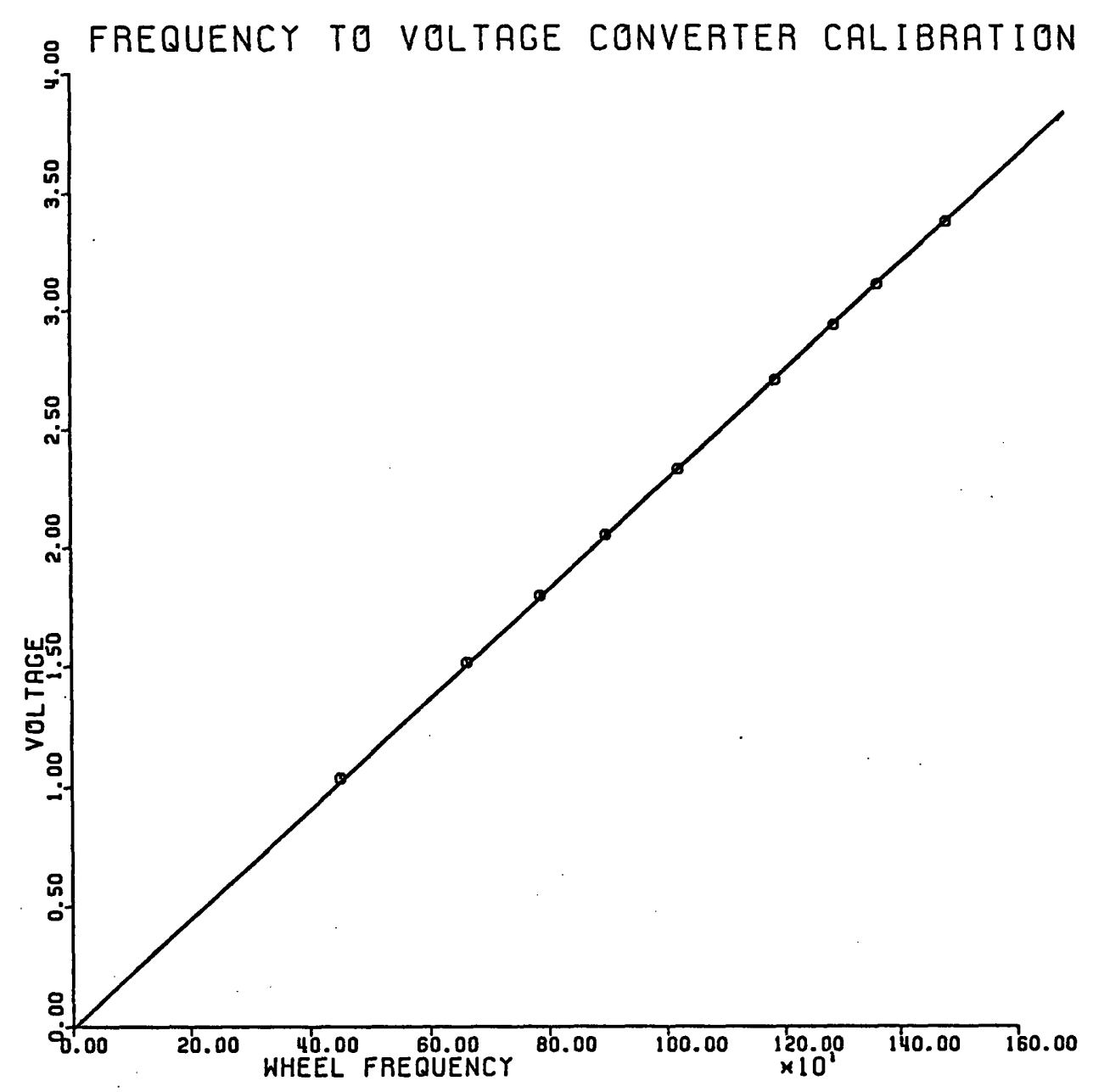

Figure 3.8: Frequency to Voltage Converter Calibration 


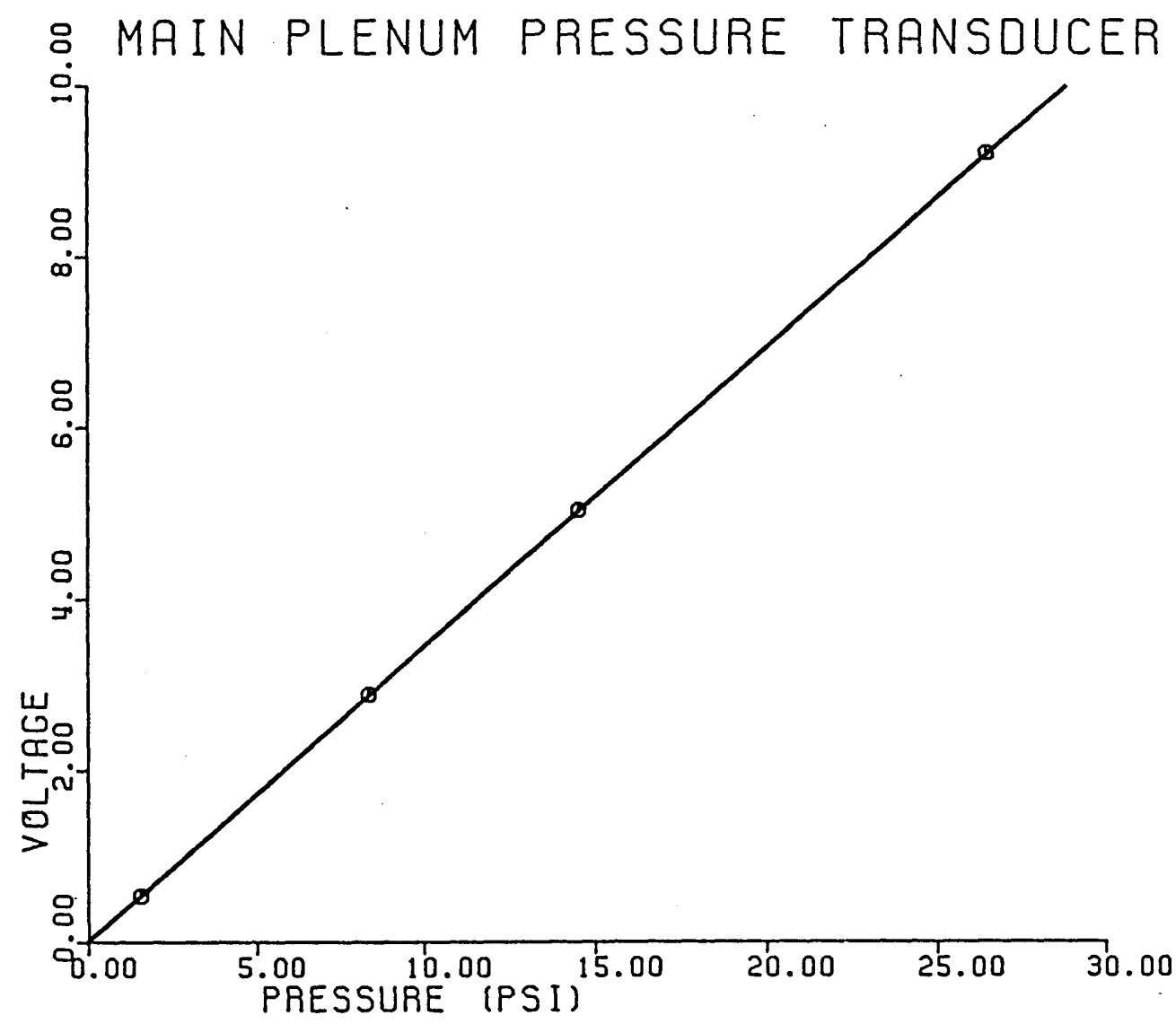

Figure 3.9: Main Plenum Pressure Transducer Calibration 


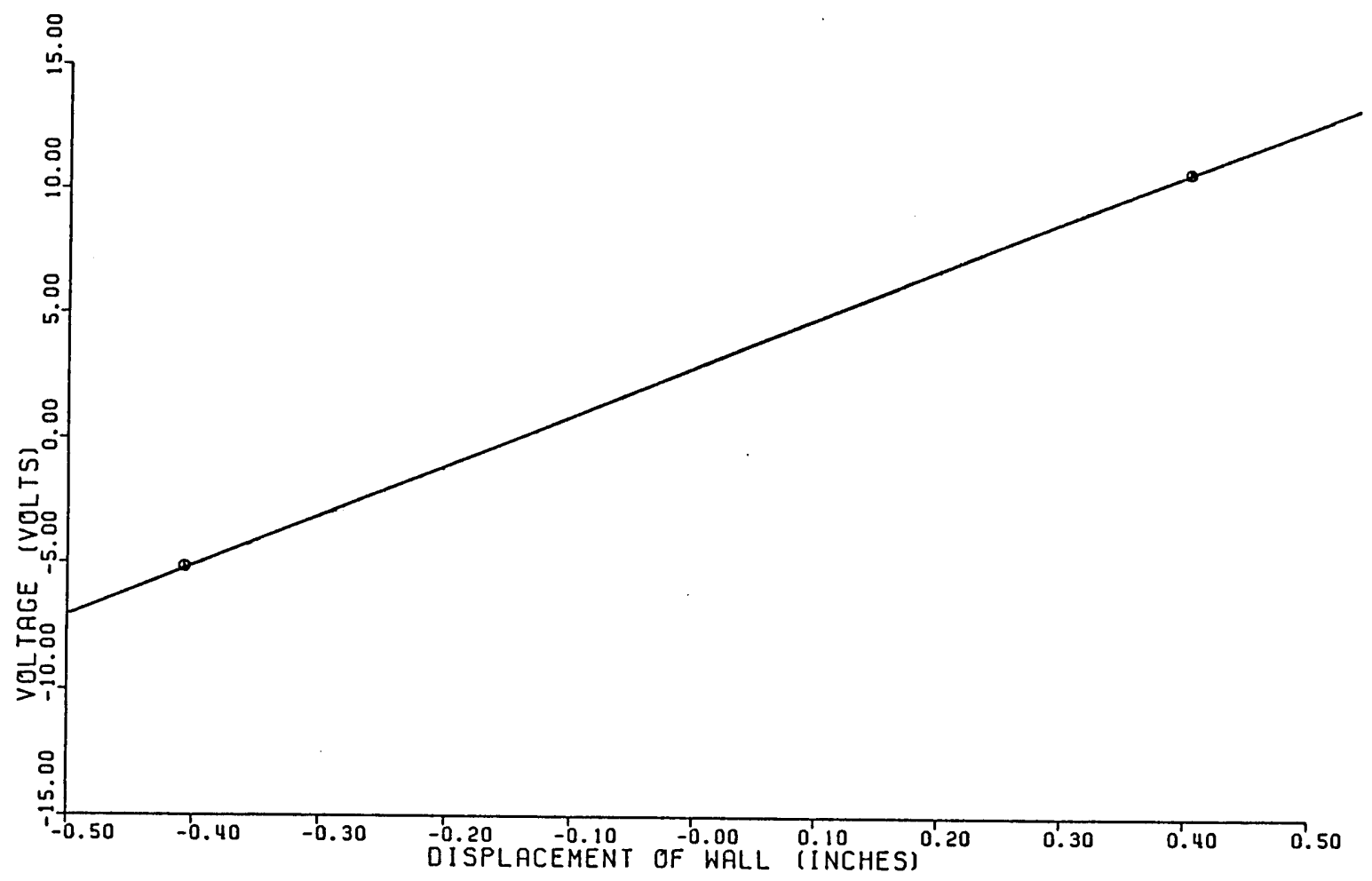

Figure 3.10: Displacement Transducer Calibration

(two point) 


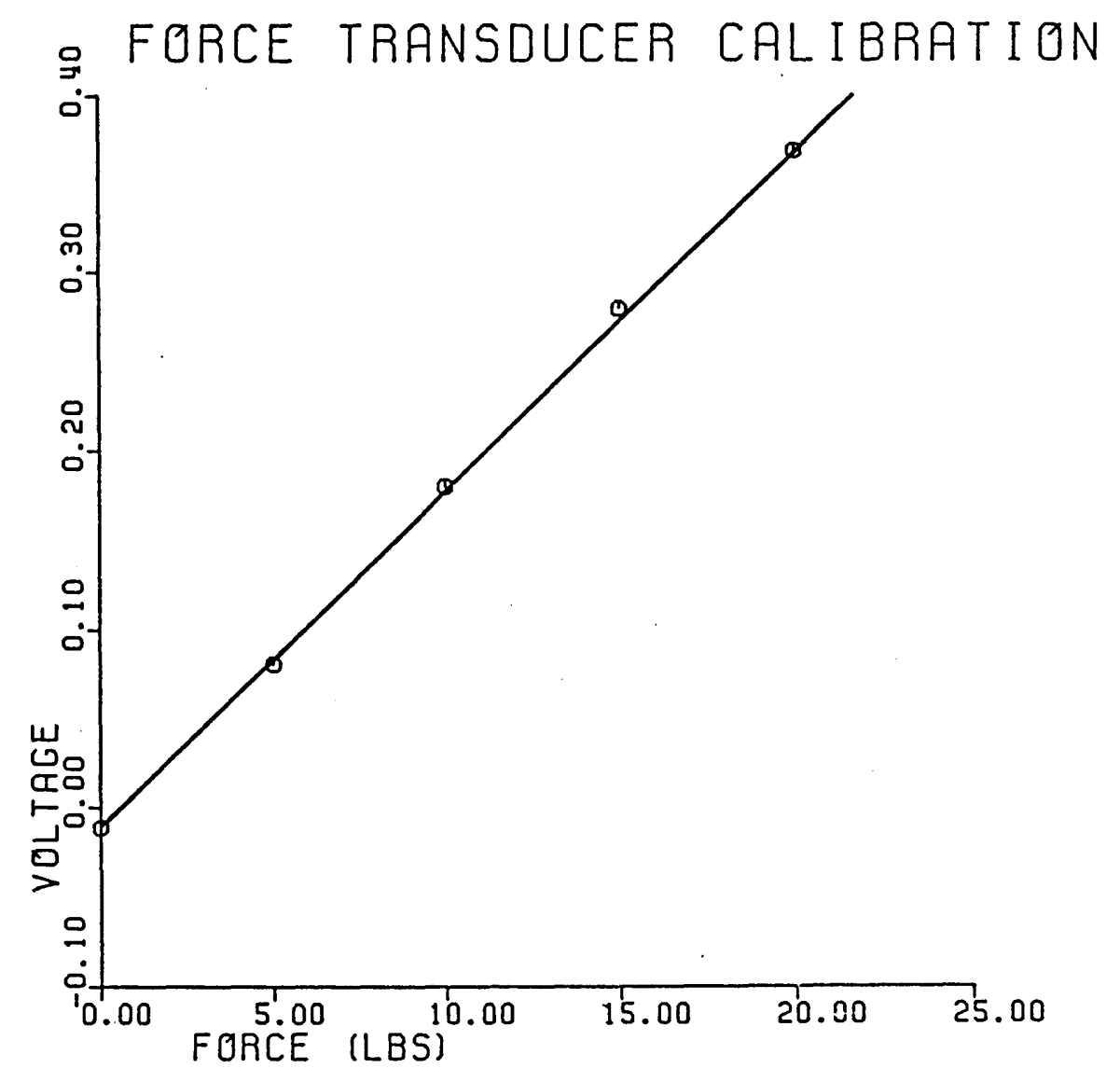

Figure 3.11: Damping Force Calibration 


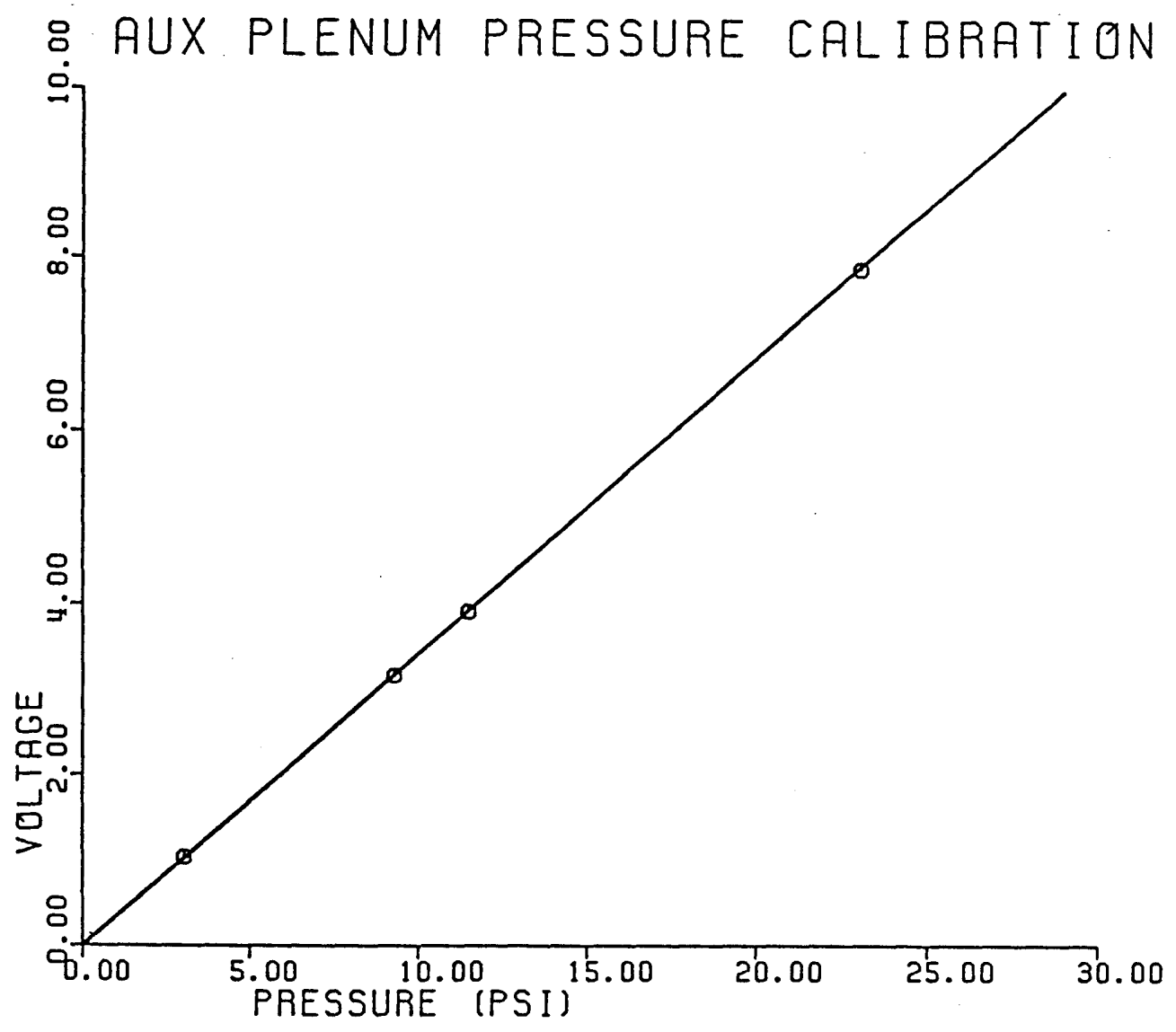

Figure 3.12: Auxiliary Plenum Pressure Calibration 


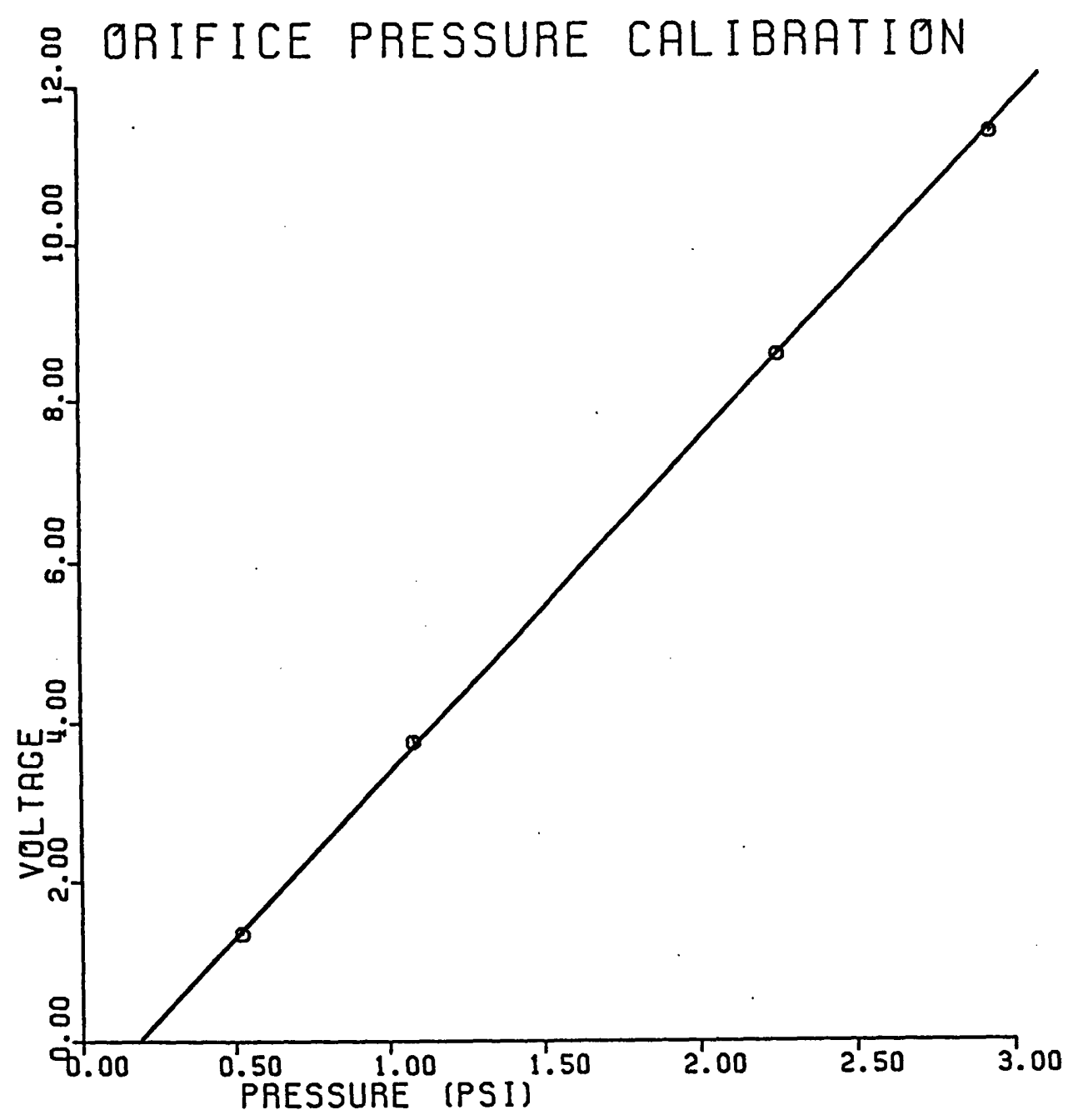

Figure 3.13: Exit Mass Flow Orifice Pressure Transducer Calibration 


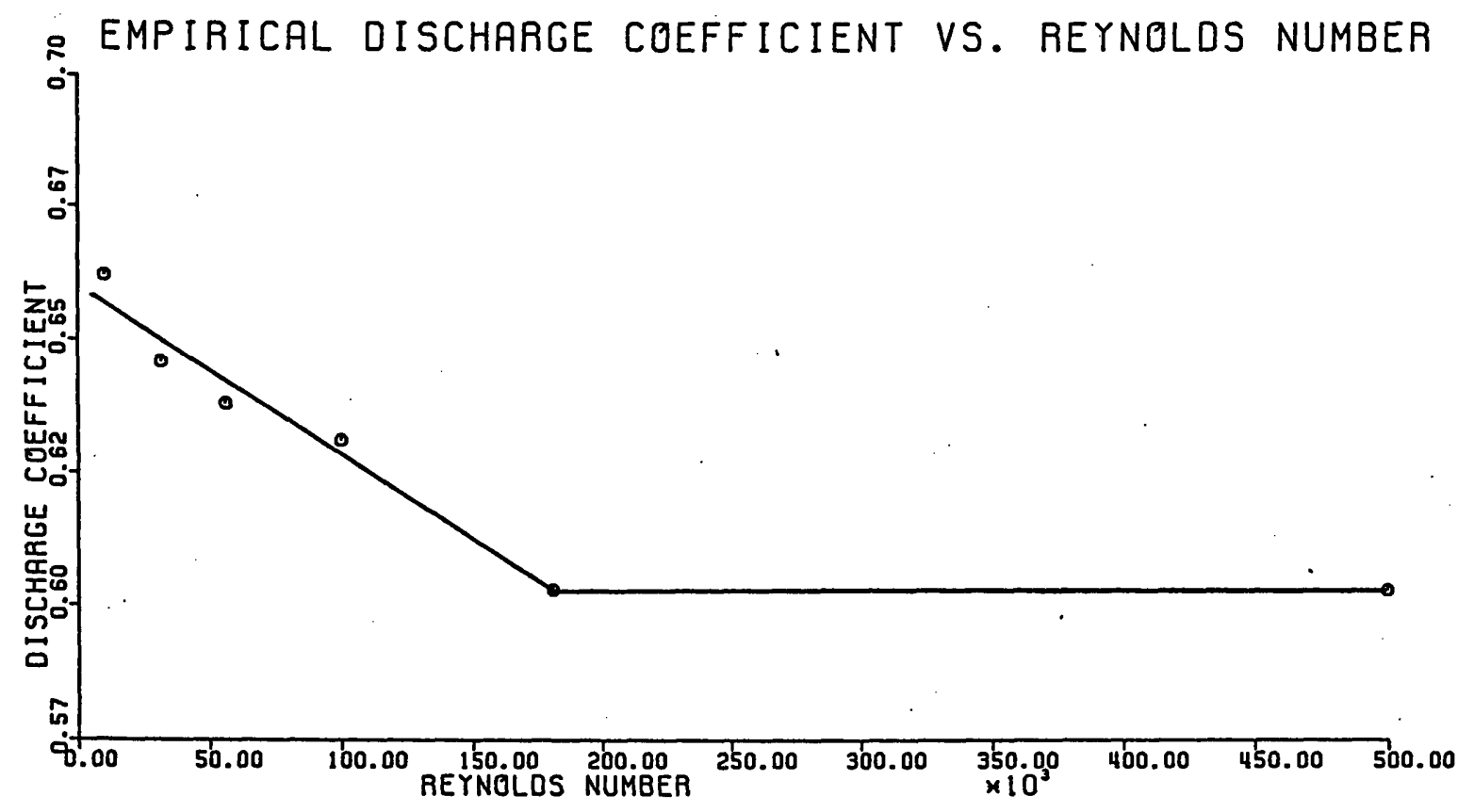

Figure 3.14: Empirical Orifice Discharge Coefficient versus

Reynolds Number 


\section{EXPERIMENTAL DATA AND ANALYSIS}

\subsection{INTRODUCTION}

The experimental phase of this investigation was divided into steady state and transient measurements. The compression system was investigated with fixed and flexible wall for three different sets of structural control parameters, at $B$ parameters ranging from 1.3 to 2.0. The steady state measurements were used to map the compression system performance and to define the surge line for both the fixed and flexible wall systems. The transient measurements were used to evaluate the model assumptions and to determine the actual performance characteristics of each component in the compression system and the flexible wall.

\subsection{STEADY STATE DATA}

\section{a. Fixed Wall System}

The experimental compression system could be operated in a fixed wall configuration by shutting the steady state pressure equalization line and bleeding the auxiliary plenum to atmospheric pressure. Under these conditions, the pressure in the main plenum forces the wall against its upper stops, yielding a fixed wall plenum.. Forcing the plenum wall against the upper stop resulted in increasing the plenum volume approximately $5 \%$, and, hence, the Bparameter $2.5 \%$. This increase had a slightly destabilizing effect on the fixed plenum wall compression system so any movement of the surge line to the left due to wall motion slightly underestimates the increase in stable flow range due solely to the movable wall.

The constant speed performance for the fixed wall system was recorded on a pressure rise versus mass flow compressor map. The compressor was operated at corrected speeds ranging from $60,000 \mathrm{rpm}$ to $100,000 \mathrm{rpm}$, corresponding to a range of B-parameters of 1.3 to 2.0. The corrected speed is based on a reference temperature of $59 \mathrm{~F}(15 \mathrm{C})$.

The speedlines were obtained by closing the steady state throttle value in small increments and recording the time averaged pressure and mass flow at each operating point. Closing the throttle valve, and hence changing the power requirements of the compressor, 
caused the steady state wheel speed to vary. To account for this, at each throttle setting, the compressed air supply to the drive turbine was adjusted to maintain constant speed. Due to the lack of precision flow regulating devices, the wheel speed could only be controlled to approximately $1 / 2 \%$ of the desired value. For this reason, pressure and mass flow measurements taken at the actual speed (as recorded by the frequency to voltage converter) were adjusted back to the intended corrected speedline. The pressure measurement was adjusted by assuming a nominal adiabatic efficiency of $65 \%$ and the non-dimensional mass flow coefficient, $\phi$, was held constant to adjust the mass flow measurements.

For the pressure adjustment, the ideal total temperature rise through the compressor was calculated assuming that the actual tangential flow velocity at the exit of the blade is given by the tip velocity times a slip factor ${ }^{16}$.

$$
\frac{T_{p}}{T_{0}}=1+\frac{(\gamma-1)}{2} \chi M_{t}^{2}
$$

The slip factor, $\chi$, is given empirically 16 by:

$$
\chi=1-\frac{2}{\# \text { of blades }}
$$

Using the definition of adiabatic efficiency:

$$
\eta_{\mathrm{th}}=\frac{\left(\frac{\mathrm{P}_{\mathrm{p}}}{\mathrm{P}_{0}}\right)^{\frac{\gamma-1}{\gamma}}-1}{\frac{\mathrm{T}_{\mathrm{p}}}{\mathrm{T}_{0}}-1}
$$

a relation between tip speed and pressure rise can be derived.

$$
\frac{\mathrm{P}_{\mathrm{p}}}{\mathrm{P}_{0}}=\left(\eta_{\mathrm{th}} \chi(\gamma-1) \mathrm{M}_{\mathrm{t}}^{2}+1.0\right) \frac{\gamma}{\gamma-1}
$$


Linearization of this relation about the measured operating point allows the pressure in the plenum to be corrected to the intended corrected speed as follows:

$$
P_{p \text { adj }}=P_{p \text { meas }}+\frac{\partial P_{p}}{\partial U}\left(U_{\text {cor }}-U_{\text {meas }}\right)
$$

where:

$$
\frac{\partial \mathrm{P}_{\mathrm{p}}}{\partial \mathrm{U}}=3.5 \mathrm{P}_{\mathrm{p}}\left(\eta_{\mathrm{Lh}} \chi(\gamma-1) \mathrm{M}_{\mathrm{t}}^{2}+1\right)^{2.5} 2 \eta_{\text {th }} \chi(\gamma-1) \frac{\mathrm{U}_{\mathrm{t}}}{\mathrm{a}_{\mathrm{p}}^{2}}
$$

The mass flow correction was calculated as follows:

$$
\dot{\mathrm{m}}_{\mathrm{adj}}=\dot{\mathrm{m}}_{\text {meas }} \frac{\mathrm{U}_{\mathrm{cor}}}{\mathrm{U}_{\text {meas }}}
$$

The compressor map for the rigid wall system is shown in Figure 4.1 , where the static pressure ratio is plotted against mass flow. The surge line shown in Figure 4.1 respresents the line where deep surge (reverse flow) occurred when the compressor was operated to the left of this line. The inlet total to plenum static pressure ratio is defined as:

$$
\pi=\frac{\mathrm{P}_{\mathrm{p}}}{\mathrm{P}_{0}}
$$

Mass flow is given in standard cubic feet per minute (SCFM).

The compressor performance is also presented in non-dimensional form as $\Psi$, the pressure rise coefficient, versus $\phi$, the mass flow parameter,in Figure 4.2. These nondimensional quantities are defined once more as:

$$
\Psi=\frac{P_{p}-P_{0}}{\rho_{0} U^{2}} \quad \text { and } \quad \phi=\frac{\dot{m}}{\rho_{0} A_{i n} U}
$$

Plotting the compressors performance in this manner tends to bring the data close to a single speedline, although, the effects of compressibility cause the speedlines to vary slightly with speed.

To aid in characterizing compression system performance during quasi-steady state operation, the root-mean-square of the pressure fluctuations was recorded at each operating 
point. The rms value of the unsteady limit cycle pressure fluctuations in the plenum, nondimensionalized by the static pressure rise, was found to generally increase as the system moved toward deep surge and it was determined to be a good indicator of the stability of the system at each operating point.

The dominant system frequency was also determined at each operating point by performing a Fast Fourier Transform (FFT) on the pressure signal and selecting the largest frequency component. This was done to determine whether the pressure fluctuations were random, or whether they could be attributed to a slightly damped system mode or limit cycle.

\section{b. Flexible Wall System}

Speedlines for the flexible wall systems were recorded in a similar manner. The experimental rig was designed to operate with various levels of wall damping, because it was of interest to operate the flexible plenum wall in the theoretically optimized configuration as well as non-optimized configurations. The variable dashpot was thus used to change the structural parameters. The wall was operated at the theoretically optimized configuration $(\mathrm{W}=.11, \mathrm{Q}=.51, \zeta=1.5)$ corresponding to the low damping constant, a slightly mistuned configuration (W=.11, $\mathrm{Q}=.51, \zeta=2.25)$ corresponding to the medium damping configuration, and a more mistuned configuration $(\mathrm{W}=.11, \mathrm{Q}=.51, \zeta=3.0)$ corresponding to the high damping configuration.

The damping constant in the dashpot was strongly dependent on both the valve setting and on temperature. For these reasons, it was not possible to calibrate the damping constant of the dashpot over long periods of time (over which the temperature varies), and it was necessary to measure and adjust the damping constant before each speedline was recorded.

The damping constant was measured by causing the rig to surge at low compressor speeds (approx. 40,000 rpm). The deep surge cycle at low speeds drove the wall periodically, but did not cause the wall to hit the displacement limiters. The wall displacement and damper force transducers thus registered a strong $10-15 \mathrm{~Hz}$ (surge frequency) oscillatory signal. The 
transient signals were recorded for 10 seconds, then processed with an FFT. Using this data, the damping constant was determined in the frequency domain for the three largest frequency components. The method used is outlined below.

We assume that the relation between the force in the damper and the velocity of the damper in the time domain is given by the following linear expression:

$$
\mathbf{F}=\mathbf{c} \dot{\mathbf{q}}
$$

Transforming the expression to the frequency domain yields the following relation.

$$
\sum \widetilde{F}_{i} e^{i \omega t}=\sum c_{i} i \omega \tilde{q_{i}} e^{i \omega t}
$$

From this expression, a damping constant can be calculated as a function of frequency using the force and displacement time traces.

$$
c_{i}=\frac{\widetilde{F_{i}}}{i \omega \tilde{q_{i}}}
$$

The steady state pressure rise versus mass flow data was recorded for each of the three flexible wall configurations. The steady state performance for these configurations, compared with the fixed wall system, is shown in Figure 4.3 . The steady state pressure rise is unaffected by the presence of the wall in the stable flow range of the rigid walled system, but, the surge line is shown moved to the left substantially. The shaded region indicates the extension in the stable flow range due to structural control. This figure shows that the flexible plenum wall is effective in suppressing surge. Also, as can be seen in Figure 4.3, the degree of surge suppression achieved is dependent on the flexible wall control parameters, as predicted. The optimized configuration performed the best, with the performance of other two configurations decreasing as the degree of mis-tuning increased. A surgeline recorded for the flexible plenum wall system with a lower than optimum damping ratio $(\zeta=.75$ ) confirmed analytical predictions that movement in any direction in parameter space away from the optimal configuration is destabilizing.

The dominant system frequencies and the non-dimensional rms value of the pressure fluctuations in the plenum were also recorded at each operating point for the flexible wall 
systems. Figure 4.4 shows the root-mean-square value of the fluctuations in plenum pressure versus mass flow coefficient for the fixed wall system and the optimized flexible plenum wall system operating at $B=1.83$ (90K speedline). On the negatively sloped region of the speedline ( above $\phi=.155$ ), the rms of the pressure fluctuations for the fixed and flexible wall system are equivalent. However, small amplitude limit cycles exist in the stabilized region, with amplitude a function of the control parameters and mass flow coefficent (primarily due to the relation with the compressor slope). The non-linear analysis described previously shows that the limit cycles in the stabilized region can be primarily attributed to the Coulomb friction in the wall and the steady state pressure equilization leakage. Small amplitude limit cycles also occur over a limited range of mass flow in the fixed wall system prior to deep surge. These small amplitude limit cycles appear to be a result of the non-linearities in the compressor and throttle characteristics.

\section{c. Comparison with Theory}

Pressure rise and mass flow are the variables used to characterize the performance of a compression system, and it is common practice to define the stability boundary on the compressor map. As mentioned previously, however, the time averaged mass flow, in itself, does not have a strong effect on system stability. The stability of a given compression system is dominated by the shape, or slope of the local compressor characteristic, not by the mass flow itself. Time averaged mass flow can only be used in defining stability through an implicit relation to the compressor slope, as defined by the compressor characteristic. The shape of these characteristics vary among compressors and also among the same compressor operating on different speedlines, even when non-dimensionalized by the dynamic head. This is demonstrated in Figure 4.2 , where the non-dimensional speedlines for the Holset turbocharger ( $60 \mathrm{~K}-100 \mathrm{~K}$ ) operating with a fixed wall are shown. The shape of each speedline, and hence the mass flow / slope relation, varies with speed, indicating that the shape of the characteristic is dependent on other parameters (predominantly Mach number.) To 
account for these effects, it is important to insure that the speedlines used for analytical predictions of the surge line on a compressor map are representative of the actual machine.

Figure 4.5 compares the 70K close-coupled characteristic for the Holset H1D turbocharger obtained by Pinsely 9 with steady data obtained for both the rigid and flexible wall systems. There is a systematic shift in the steady state mass flow between the data obtained in this research and Pinsley's data. However, with this considered, the extension of the speedlines due to the flexible plenum wall has the same effect on steady state system performance as lowering the B-parameter of the compression system. This demonstrates that the flexible wall suppresses surge with negligible performance losses.

Although the maximum controllable slope stability criteria is not sensitive to small changes in the specific characteristic used, the predicted mass flow at instability can be greatly affected by small variations in the shape of the characteristic. In fact, the predicted mass flow at instability can vary upwards of $10 \%$ depending on the method used to curve fit the characteristic from the same set of experimental data. Despite this, the surge line on a pressure ratio versus mass flow performance map is still a common method to assess the effectiveness of a surge control method.

Figure 4.6 shows the predicted and the experimentally determined surge lines for the rigid wall and optimized system. The predicted surge line is based on the linear instability point as determined by the eigenvalue stability analysis described previously. The compressor characteristics used in this analysis resulted from a 3rd degree polynomial curve fit of the closecoupled speedlines of the Holset turbocharger measured by Pinsely ${ }^{9}$ shown in Figure 4.7.

Although the $\Psi, \phi$ relation is not exact and non-linear terms are not considered, the predicted and experimental surge lines are in reasonable agreement. The same results are plotted in non-dimensional form as predicted versus experimental mass flow coefficient at surge for the fixed wall and optimized system in Figure 4.8 .

The flexible plenum wall also demonstrated the ability to suppress deep surge if the wall was freed by equalizing the pressure in the aerodynamic spring to the main plenum 
pressure during a rigid wall surge cycle. This demonstrated in Figure 4.9, where fixed wall surge is suppressed by equalizing the auxiliary plenum pressure, thus freeing the wall from the displacement limiters. The time traces in Figure 4.9 show the wall bouncing on the stops as the auxiliary plenum pressure equalizes. As shown, when the wall is free from the stops, the deep surge cycle is suppressed. Although not shown, the wall continues to move towards its steady state equilibrium position as the pressure in the auxiliary plenum continues to equalize. This ability to suppress surge in a highly non-linear regime was also predicted by the nonlinear analysis. Similar behavior was also found separately by both Pinsely ${ }^{9}$ and Huang 8 with their respective (linearly designed) control schemes.

The experimental results can be compared to the non-linear analysis by comparing the amplitudes of pressure fluctuations in the plenum as a function of mass flow. The pressure fluctuations with a dominant frequency near the Helmholtz frequency were determined to be small amplitude limit cycles and not the result of random system excitation.

As input to the non-linear calculation, the friction force present during wall motion was measured to be approximately 10 newtons and the leakage was estimated to be equivalent to a .003 meter diameter orifice plate. The $3 \mathrm{rd}$ order fit of the $90 \mathrm{~K}$ speedline was used as for the compressor characteristic. The results of the non-linear calculation and the experiment for the optimized system operating at $B=1.83$ are shown in Figure 4.10 , where the amplitude of the small plenum pressure limit cycles before the onset of deep surge are shown versus mass flow coefficient. The linear stability boundary is also shown in Figure 4.10 for comparison. Although the linear analysis could not predict the small amplitude limit cycle behavior in the stabilized region, it was able to accurately predict the deep surge line. This indicates that the small amplitude limit cycles in the stabilized region are the result of non-linear effects not modelled in the linear analysis and that, if theses effects are small, the linearly predicted surge line corresponds to deep surge. The non-linear analysis accurately predicts limit cycles in the stabilized region, however, the detailed relationship between mass flow and limit cycle amplitude is not captured. This indicates that the 3rd degree curve fit of the quasi-steady 
compressor characteristic is not an exact relationship between steady state mass flow and slope.

\subsection{TRANSIENT SYSTEM BEHAVIOR}

The steady state data demonstrated that a properly tuned, flexible plenum wall can stabilize surge and shift the surge line significantly to the left on a compressor map. The steady state data also demonstrated that the lumped parameter model can be applied sucessfully to the flexible wall system. However, to fully investigate the system performance and the assumptions made in modelling the system, time resolved, unsteady data is required. Time resolved measurements were thus recorded for the fixed wall compression system and for the three control parameter configurations at the $70 \mathrm{~K}$ and $90 \mathrm{~K}$ speedlines corresponding, to $\mathrm{B}$ parameters of 1.45 and 1.83 .

\section{a. Fixed Wall System}

As noted previously, the flow through the compression system becomes progressively more unsteady as the system approaches the surge line. To demonstrate this, the time resolved non-dimensional mass flow coefficient and the non-dimensional pressure rise for the fixed wall system are shown in Figure 4.12 for 5 operating points at a B-parameter of 1.45 corresponding to points on the $70 \mathrm{~K}$ speedline. The transient mass flow measurements are based on a linearized hot wire calibration and therefore, the large oscillations in mass flow are presented for qualitative information only. The data shown correspond to points in both the stable and unstable operating regions. The 5 operating points, A-E, are shown on the compressor operating map in Figure 4.11.

Points A and B are on the negatively sloped region of the speedline and exhibit steady, stable operation with only slight unsteadiness.

Point $\mathrm{C}$ is slightly to the left of the peak of the compressor characteristic (peak is at $\phi=$ $.120)$ and is exhibiting a small amplitude limit cycle (mild surge). The frequency of the mild 
surge cycle is approximately $14.5 \mathrm{~Hz}$, compared to the predicted Helmholtz frequency of 17.5 Hz.

Points $\mathrm{D}$ and $\mathrm{E}$ are in deep surge. The time averaged pressure rise and mass flow are decreased and the frequency of the deep surge oscillations has been changed to approximately $10 \mathrm{~Hz}$. Since the hotwire cannot distinguish reversed flow, the reversed flow regions are shown with a dashed line. The decrease in frequency is due to non-linearities associated with plenum blow-down and re-pressurization as described by Fink 1 .

The surge cycles for points $\mathrm{C}$ and $\mathrm{E}$, plotted as non-dimensional pressure rise versus mass flow, are shown in Figure 4.13. As shown, the limit cycles grow in amplitude as the compressor approaches the deep surgeline. The compressor is shown to orbit its timeaveraged operating point on the compressor's steady state characteristic primarily due to the inertia of the fluid in the inlet duct. For both mild and deep surge, the variation in $\phi$ is much larger than variation in $\Psi$.

The Fourier transform of the pressure signal for an operating point located between points $B$ and $C,(\phi=.121)$, is shown in Figure 4.14 . The system has a narrow band frequency spectrum, dominated by the mode near the Helmholtz frequency, supporting the validity of the one-mode lumped parameter model.

\section{b. Flexible Wall System}

The transient behavior of the flexible wall system was somewhat similar to the fixed wall system, although surge occured at lower mass flow coefficient. Small amplitude limit cycles existed over the entire stabilized region. The amount of wall motion required to suppress surge in the stabilized region is a strong function of the non-linearities in the flexible wall control system such as friction and leakage.

The unsteady behavior of operating points on the $70 \mathrm{~K}$ and $90 \mathrm{~K}$ speedlines were similiar, however, since more data was recorded at the lower speed, the $70 \mathrm{~K}$ data is presented. Figures 4.15A and 4.15B show the non-dimensional mass flow, pressure rise, and 
corresponding non-dimensional wall motion for the medium damping configuration operating at the $70 \mathrm{~K}$ speedline $(\mathrm{B}=1.45)$ for 5 operating points, F-J shown on the compressor map in Figure 4.12.

Point $F$ is in smooth operation on the negatively sloped region of the map. The steadystate and unsteady behavior is similar to points $\mathrm{A}$ and $\mathrm{B}$ for the fixed wall configuration. The position of the wall, as shown in Figure 4.15B , shows the wall to be stationary, indicating that the ambient and internal disturbances in the stable system are not large enough to overcome the wall friction.

Points G, H, and I are located on the positively sloped, stabilized region of the characteristic and exhibit small amplitude limit cycles. The non-dimensional wall motion, shown in Figure 4.15B, demonstrates that the wall is exhibiting a small amplitude limit cycle at the system frequency. As shown, the maximum wall motion required to stabilize the system is approximately $0.1 \%$ of the plenum volume. The power dissipated by the flexible plenum wall during limit cycles of this order is approximately $0.05 \%$ of the steady power of the compression system.

The reduced amplitude of the limit cycles for points $\mathrm{H}$ and I are due to the 'hump', or flattening, near the peak of the speedline as shown in Figure 2.1. This 'hump' is not captured in the 3rd degree curve fit of the steady-state data and is, therefore, not in the analytical results. The unsteady time traces demonstrate that the amplitude of the limit cycle is dependent on the local compressor slope and not directly dependent on the mass flow.

At point $\mathrm{J}$, deep surge, the pressure and mass flow traces are similar in amplitude to those with the fixed wall although the fluctuations have a much lower natural frequency (4-5 $\mathrm{Hz}$ ). Also, in deep surge, the wall is shown to be hitting the displacement limiters, indicated by the flat spots on the time trace of the wall motion.

The surge cycles for points $\mathbf{G}$ and $\mathrm{J}$ are plotted as pressure rise versus mass flow in Figure 4.16. As with the fixed wall surge cycles, the reversed flow regions are indicated with 
a dashed line and are presented for qualitative information only. The surge cycle is simliar in behavior to the fixed wall surge cycle shown in Figure 4.13.

The Fourier transform of the pressure signal for point $I$ is shown in Figure 4.17. Here again, the signal is narrow banded about the Helmholtz frequency, however, there are now two peaks in the spectrum, indicating the two modes of the system.

\section{c. Comparison to Theory}

The time traces of limit cycles obtained in the stabilized region of the compressor map can be compared to the time domain response calculated using the non-linear equations of motion. Figure 4.18A, shows both the experimental and calculated time resolved nondimensional pressure rise and mass flow coefficients for a stabilized operating point. The medium damping configuration is shown operating at $B=1.83$ and $\phi=.128$ on the $90 \mathrm{~K}$ speedline. A Coulomb friction term of $F=.002$ (corresponding to $10 \mathrm{~N}$ ) and an equivalent leakage orifice of $.003 \mathrm{~m}$ diameter was used as input to the calculations. The unsteady data non-dimensional pressure oscillations have an rms value of $0.7 \%$ at approximately $16 \mathrm{~Hz}$. The predicted non-dimensional pressure fluctuation limit cycle has a rms value of $0.4 \%$ at approximately $19 \mathrm{~Hz}$.

The predicted and experimental responses are in good agreement, when the simplicity of the model is considered. However, as mentioned previously, the surge dynamics of the system are not strongly related to the steady state mass flow coefficient, but rather to the compressor slope. Therefore, a better comparison of actual versus predicted response would be obtained by matching the amplitude of the pressure oscillations in the limit cycle, since the amplitude of the limit cycle was shown to be strongly dependent on compressor slope.

Figure $4.18 \mathrm{~B}$, shows the predicted response of the same system at a $3 \%$ lower mass flow coefficient, $\phi=.124$, yet a $30 \%$ larger positive compressor slope. At this operating point, a limit cycle of $0.7 \%$ is predicted at a frequency of $18 \mathrm{~Hz}$. The amplitude of the wall motion motion for both the predicted and measured $0.7 \% \mathrm{rms}$ pressure limit cycles is shown in Figure 
4.19. As shown, the amplitude of the predicted wall motion (approximately $0.1 \%$ of plenum

volume) agrees with experimental results indicating that the analysis does accurately model the coupling between the wall mass-spring-damper system and the basic compression system.

\subsection{VALIDATION OF ASSUMPTIONS}

\section{a. Quasi-steady Compressor Slope}

One of the major assumptions used in modelling the compression system is that the compressor remains on its steady state characteristic during transient operation of frequencies on the order of the Helmholtz frequency. The quasi-steady characteristic assumption has been used by many other researchers $1,4,5$. The degree to which this is valid has been investigated by others 8,11 , notably Fink ${ }^{1}$, who correlated experiment and analytical results by introducing a first order time lag to the steady-state characteristic. Fink determined that the time lag should be on the order of the flow through time of the compressor. Huang 8 fit a compressor resistance term needed to equate the measured unsteady compressor slope to the steady slope obtained from a curve fit through steady data. However, in developing each of the correlation methods the researchers based the correction terms on the steady state slope determined from a curve fit method, which, as described previously, is only an estimate of the quasi-steady slope.

Here, the unsteady pressure rise versus mass flow relation is calculated directly from the unsteady data and compared to the quasi-steady slopes. The unsteady data was calculated from operating points exhibiting small amplitude limit cycles. The instantaneous pressure rise versus mass flow slope was determined by applying the inlet duct momentum equation and using a measured transfer function between the inlet mass flow and plenum pressure. Since we did not try to excite the system at different frequencies, the transfer function could only be accurately determined at or near the system Helmholtz frequency where enough frequency component existed for an acceptable signal to noise ratio. The transfer function was thus only determined over a small, but relevant, range of frequencies. 
The compressor inlet mass flow to compressor exit pressure transfer function is related to the slope of the instantaneous compressor slope through the inlet momentum equation.

$$
\frac{\partial \Delta \mathrm{P}_{\mathrm{c}}}{\partial \dot{\mathrm{m}}} \delta \dot{\mathrm{m}}_{1}-\delta \mathrm{P}_{\mathrm{p}}=\rho_{0} \mathrm{~L}_{\mathrm{c}} \frac{\partial \mathrm{C}_{\mathrm{x}}}{\partial \mathrm{t}}
$$

non-dimensionalizing and solving for the slope yields:

$$
\frac{\partial \Psi}{\partial \phi}=\frac{1}{\rho_{0} U} \frac{\partial P_{p}}{\partial C_{x}}+i \omega \frac{L_{c}}{U}
$$

The compressor slope, as measured yields a real and imaginary part.The real part of the transfer function represents the instantaneous compressor characteristic. The imaginary part can be viewed as a lag term, similar to the lag term developed by Fink. The real part of the transfer function is plotted versus steady state mass flow coefficient for the $70 \mathrm{~K}$ and $90 \mathrm{~K}$ speedlines in Figure 4.20. The compressor slopes plotted in Figure 4.20 contain slopes measured from both rigid and flexible wall systems, indicating that the presence of the wall has no noticeable effect on the instantaneous compressor slope. Also plotted in this figure, are the quasi-steady slopes resulting from two methods of fitting the same steady state data. One method shown is the derivative of a 3rd order polynomial curvefit, the other is a 3rd order polynomial fit of the derivative of a cubic spline fit of the steady state compressor data. As shown, the quasi-steady slopes and the compressor slopes determined from unsteady data are in reasonably good agreement. It should be noted that the unsteady compressor slope falls within the variance between the two quasi steady slopes calculated by curve fits. Therefore, the quasi-steady assumption was determined to be an adequate representation of the unsteady compressor slope over the range of flow coefficients investigated.

The time lag for both the $70 \mathrm{~K}$ and $90 \mathrm{~K}$ speedlines was approximately 5 - 10 milliseconds, corresponding to $.065-.13$ Helmholtz resonator periods. The compressor through flow time was determined to be approximately 3.0 milliseconds. The lag term is thus on the order of the through time of the compressorand it seems reasonable to attribute the lag to 
unsteady aerodynamic effects within the compressor passages. This is in agreement with the conclusions of Fink $^{1}$, who determined that a lag term on the order of compressor through flow time was needed for agreement between the predicted and experimental behavior of a compression system in deep surge. However, Fink found that a lag term of this order should have a negligible effect on system stability over the range of B-parameters investigated. The results of this study thus indicate that the quasi-steady compressor slope is adequate for predicting the onset of surge.

\section{b. Assumption of Constant Wheel Speed}

Another basic assumption used in modelling the compression system was the assumption that wheel speed remains constant for perturbations in mass flow and pressure rise. This is generally not valid since pressure and mass flow perturbations vary the power requirements of the compressor causing the wheel to also vary with these oscillations, assuming constant drive torque from the turbine. Fink ${ }^{1}$ assessed the constant wheel speed assumption by modelling a variable wheel speed compression system. Variable wheel speed was shown to have a stabilizing effect on the compression system; the degree of stabilization was shown to be a function of the A-parameter. Using this analysis, the effect of variable wheel speed on the stability of the compression system at B-parameters of $1.3-2.0$ was determined to be negligible. The unsteady data was used to evaluate this prediction and to also investigate the effect of variable wheel speed during deep surge.

Figure 4.21A shows the wheel speed and pressure fluctuations for the fixed wall system operating near the surge line in a small amplitude limit cycle. The B-parameter is 1.83 and the mass flow coefficient is $\phi=142$. If the wheel speed and pressure fluctuations were strongly coupled, the pressure and wheel speed variations would scale as follows:

$$
\frac{\mathrm{P}_{\mathrm{p}}+\delta \mathrm{P}_{\mathrm{p}}}{\rho_{0} \overline{\mathrm{U}}^{2}} \approx \frac{\rho_{0}(\mathrm{U}+\delta \mathrm{U})^{2}}{\rho_{0} \overline{\mathrm{U}}^{2}}
$$




$$
\text { or } \quad \delta \Psi \approx 2 \frac{\delta U}{\bar{U}}
$$

However, for the $\mathbf{2 . 4 5 \%} \mathrm{rms}$ pressure fluctuations, the corresponding wheel speed fluctuations of $0.2 \%$ are an order of magnitude below the wheel speed fluctuations required by the above scaling law. Therefore, the assumption of constant wheel speed was determined to be adequate for modelling the small perturbation response of the compression system.

Time traces of the wheel speed and pressure fluctuations for deep fixed wall surge cycles $(B=1.83 ; \phi=.105)$ are shown in Figure 4.21B. Variable wheel speed was shown by Fink ${ }^{1}$ to have a large effect on the deep surge behavior of some compression systems, dependent on the A and B parameters. For the ranges of parameters used in this investigation, wheel speed was shown to have a negligible effect on deep surge. For a typical deep surge cycle, with rms pressure fluctuations of $10 \%$, the wheel speed fluctuations were only approximately $0.5 \%$, also shown in Figure $4.21 \mathrm{~B}$.

\subsection{COMPONENT PERFORMANCE}

The unsteady data was also used to determine the dynamic behavior of the wall. In the analytical model, the wall was modelled as a linear mass-spring-damper system. By analyzing the unsteady data during large and small amplitude surge cycles, the non-linearities in the spring and damper could be evaluated.

\section{a. Aerodynamic Spring}

The air in the aerodynamic spring used in the experimental rig was assumed to behave isentropically. Using the isentropic assumption and gas properties for air, a linearized spring constant can be derived. For a closed chamber this linearized spring constant is given by:

$$
k=\frac{\gamma P_{p} A_{p}^{2}}{V_{\text {aux }}}
$$


To assess the linearity of the aerodynamic spring, the spring restoring force was plotted against wall displacement. The results for the medium damping constant system operating in deep surge at $B=1.83$ are shown in Figure 4.22 . The response of the spring is essentially linear with the exception of regions where wall is hitting the displacement limiters. The spring constant measured from Figure 4.22 , is approximately $24,000 \mathrm{n} / \mathrm{m}$. Using the isentropic relation given above, the spring constant was predicted to be $27,000 \mathrm{n} / \mathrm{m}$.

A spring constant could also be determined from Fourier analysis of the unsteady data. The spring constant was determined by evaluating the ratio of the Fourier components of the auxiliary plenum pressure and the wall displacement signal at the dominant system frequency. Explicitly:

$$
\mathbf{k}_{\mathrm{i}}=\frac{\widetilde{\mathrm{F}_{\mathbf{i}}}}{\widetilde{\mathrm{q}}_{\mathrm{i}}}
$$

The spring constant calculated from the measured transfer function is shown as a function of frequency in Figure 4.23. The Fourier spectrum of the wall motion used in this calculation is also shown in Figure 4.23. As shown, the measured transfer function is reasonably constant for frequencies with significant Fourier components. Those frequencies not excited by system dynamics yield erroneous result due to the low signal to noise ratio, however, the frequencies with significant excitation yield a spring constant of approximately $24,000 \mathrm{n} / \mathrm{m}$, in agreement with the spring constant calculated using the time domain approach.

\section{b. Dashpot Behavior}

The damping constant can be determined in a similar manner as the spring constant. As noted previously, the damping constant for each speed line was measured during a deep surge cycle at low speed. By measuring the damping constant during large amplitude periodic motion, the effect of the sliding friction on the Fourier analysis is minimized. However, the behavior of the dashpot during small amplitude motion determines the effectiveness of the 
control scheme. This behavior can be examined if the effect of the constant amplitude Coulomb friction force is considered.

Figure 4.24 shows a sample of the damper force and the wall displacement signals during a small amplitude limit cycle. The pressure fluctuations in the limit cycle correspond to $0.5 \%\left(\Delta \mathrm{P}^{\prime} / \mathrm{P}_{\mathrm{c}}\right)$ rms limit cycle. The damping constant calculated from the transfer function of these two signals is shown as a function of frequency in Figure 4.25. Also shown is the frequency spectrum of the displacement signal. Again, for frequencies with significant excitation, the results are quite consistent. For frequencies near $15 \mathrm{~Hz}$, the damping constant is measured to be approximately $1700 \mathrm{n} \mathrm{s} / \mathrm{m}$. A similar analysis was performed for a larger, $2 \%$ rms pressure fluctuation limit cycle with the same damping characteristics and the damping constant was determined to be $1600 \mathrm{n} \mathrm{s} / \mathrm{m}$. The damping constant measured for this operating condition from a large amplitude limit cycle was $1500 \mathrm{n} \mathrm{s} / \mathrm{m}$. This decrease in calculated damping constant with increasing limit cycle amplitude agrees with the predicted effect of the constant amplitude friction force in the damper on the damping constant determination.

\subsection{SUMMARY OF RESULTS}

The results of the experiment investigation of surge suppression using a tailored flexible plenum wall show that the flexible plenum wall is an effective technique for suppressing centrifugal over a wide range of operating conditions. The modified compression system extended the stable operating range 25 to 30 percent in flow while increasing the maximum tolerable compressor slope by an order of magnitude. There was no noticeable decrease in compression system performance as a result of the flexible plenum wall control scheme. The effectiveness of the flexible plenum wall was also shown to be a function of the non-dimensional control parameters, as predicted.

Linear and non-linear analyzes both gave useful descriptions of unsteady system behavior. The linear model showed trends in stability limits as functions of control and compression system parameters. It also gave good agreement with the measured surge line, 
within limits of the compressor characteristic discussed previously. The non-linear analysis predicted the existence of limit cycles in the stabilized region and was used to demonstrate that small amplitude limit cycles occured primarily due to Coulomb friction and leakage. The nonlinear analysis was crucial for setting limits on the non-linearities to insure that the linear analysis was adequate to predict the flexible plenum wall's performance.

The unsteady data validated many of the assumptions used in modelling the compression system. Compressor transfer functions, measured at or near the Helmholtz frequency, showed that the quasi-steady compressor characteristic was adaquate for modelling the compression sytem. The compressor transfer function revealed the existence of a small lag term, on the order of through flow time. However, a lag term on this order was determined to have a negligible effect on compressor stability. Time traces of the wheel speed during mild and deep surge cycles showing the wheel speed to remain essentially constant, validated the conclusions from Fink's analysis ${ }^{1}$ on assessing the effect of finite wheel inertia using the Aparameter.

The unsteady data was also used to characterize the performance of the flexible wall itself. By analyzing the Fourier content of the wall displacement and force in the damper signals, the dashpot was shown to to have a constant damping constant over the range of frequencies with a significant Fourier component. The aerodynamic spring was also shown to behave linearly, as modelled. 


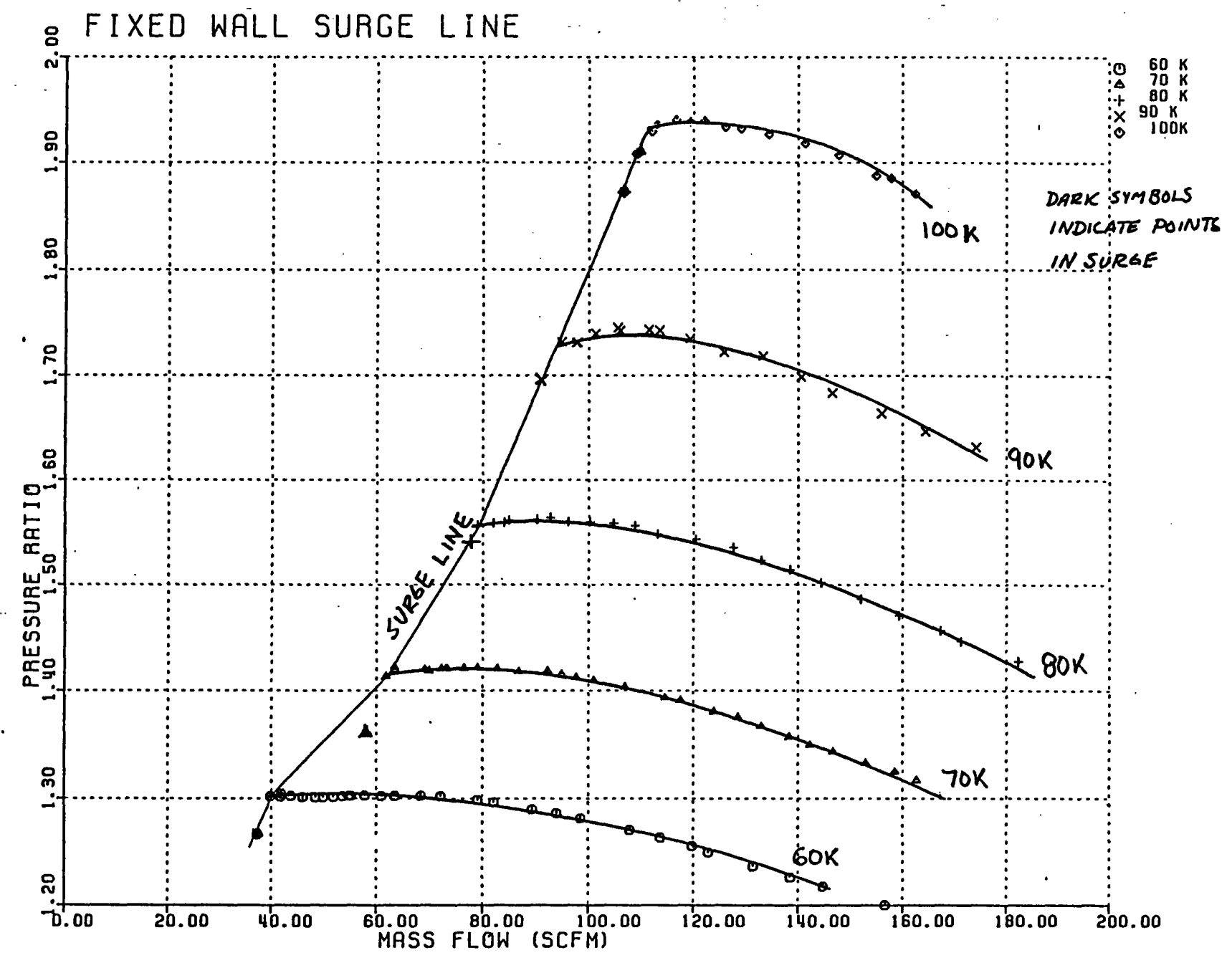

Figure 4.1: Fixed Wall Compressor Performance Map 


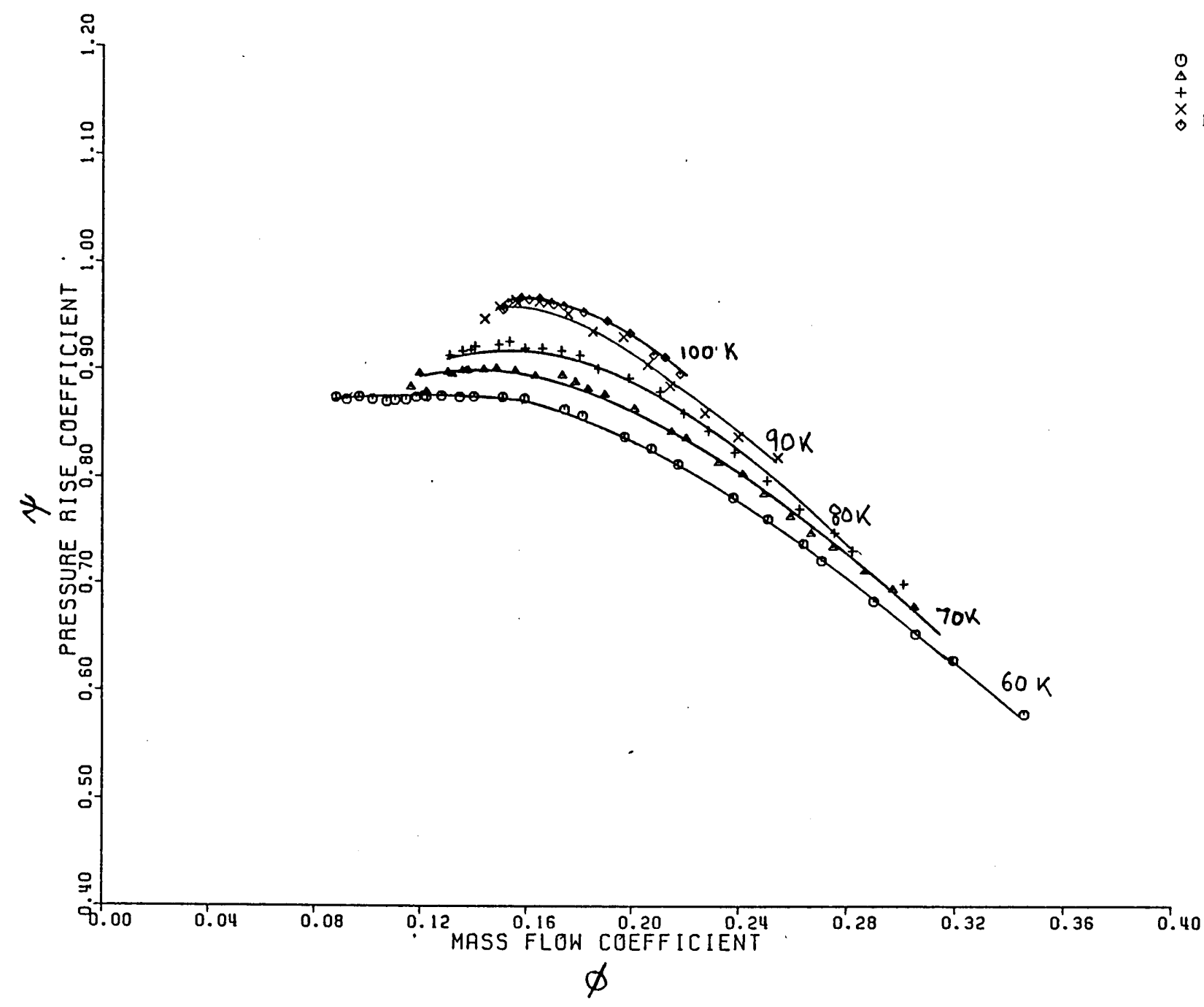

Figure 4.2: Non-dimensional Fixed Wall Performance Map 


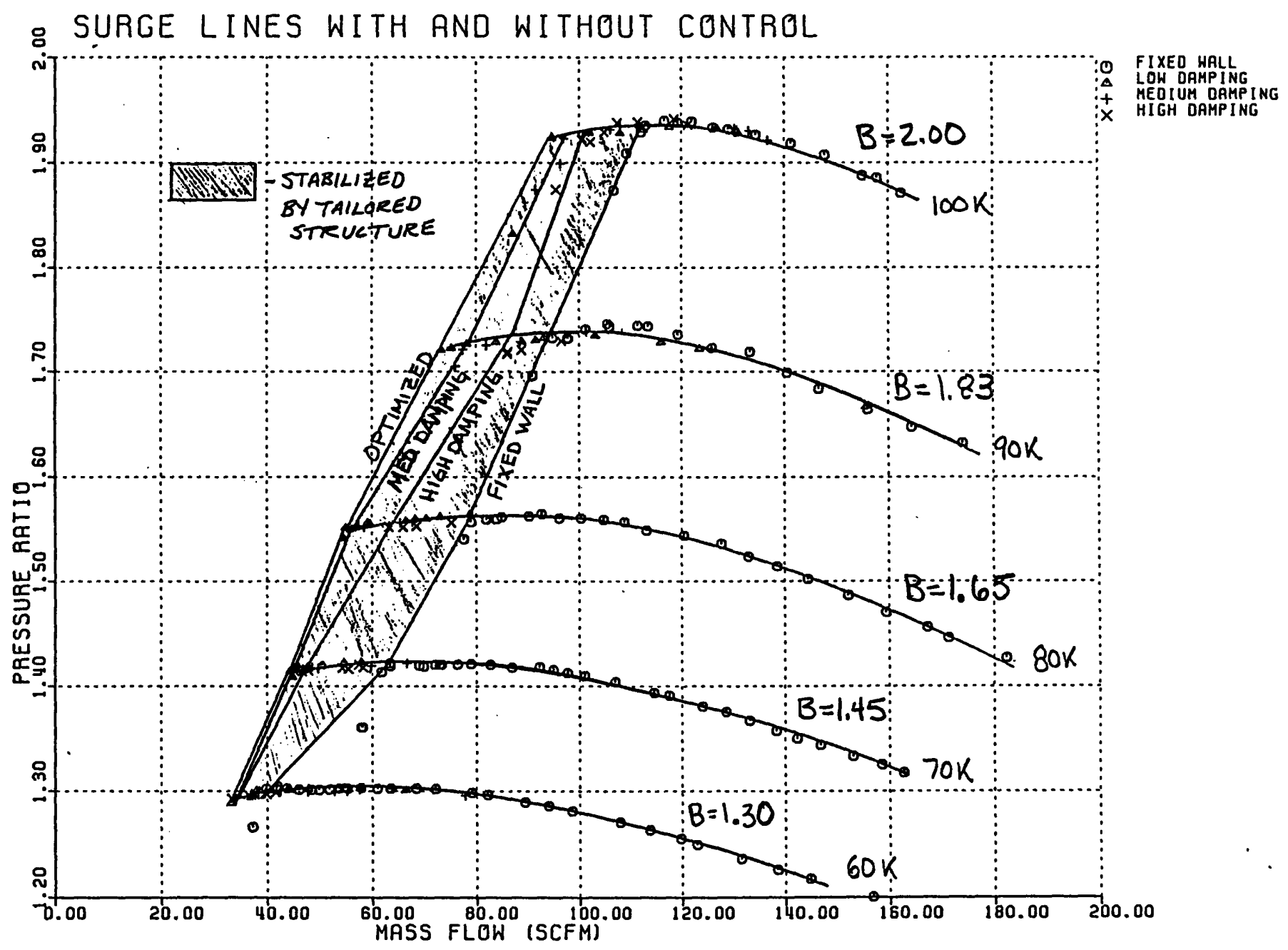

Figure 4.3: Compressor Performance Map

Fixed and Flexible Wall

95 


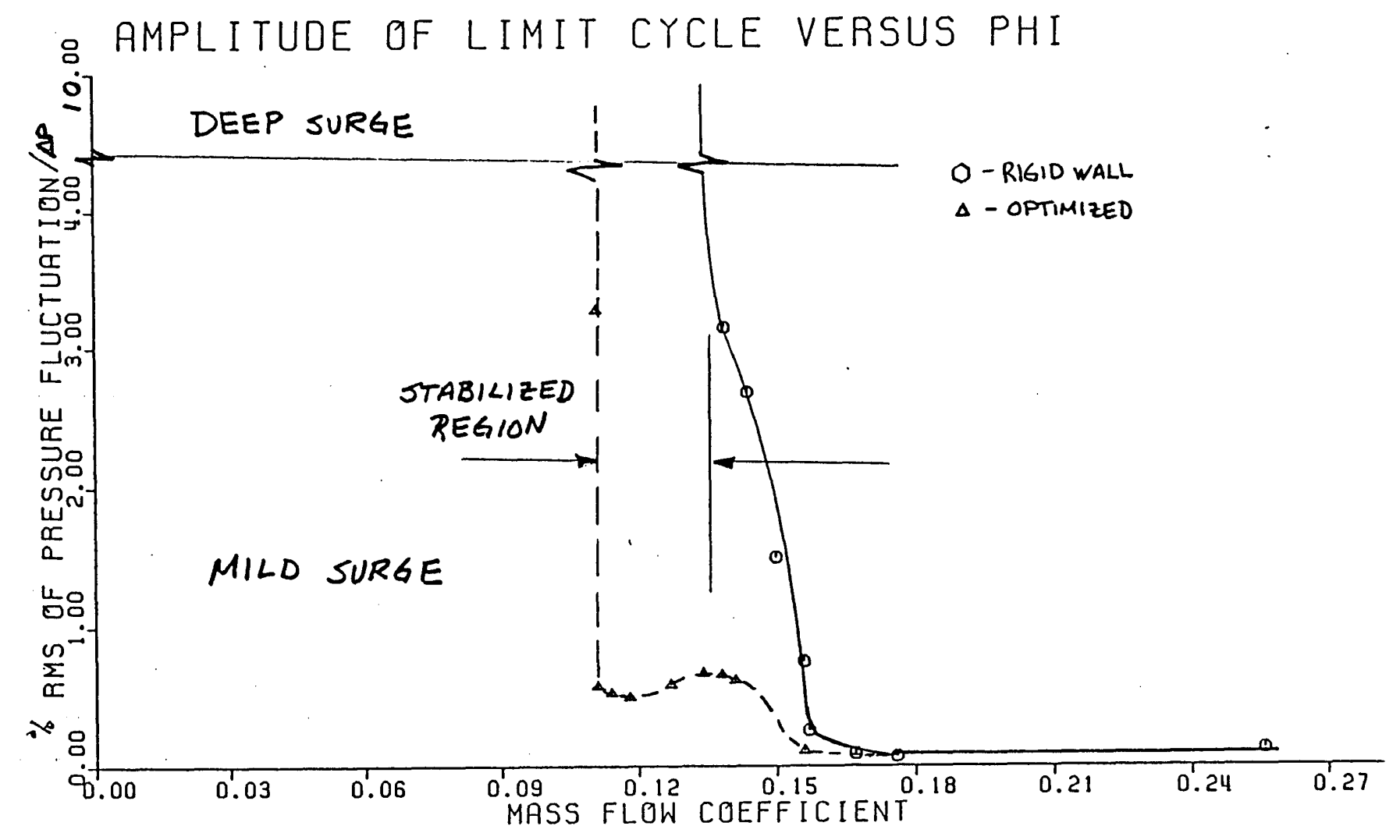

Figure 4.4: Amplitude of Limit Cycle in Stablized Region

$$
\text { ( } B=1.83)
$$




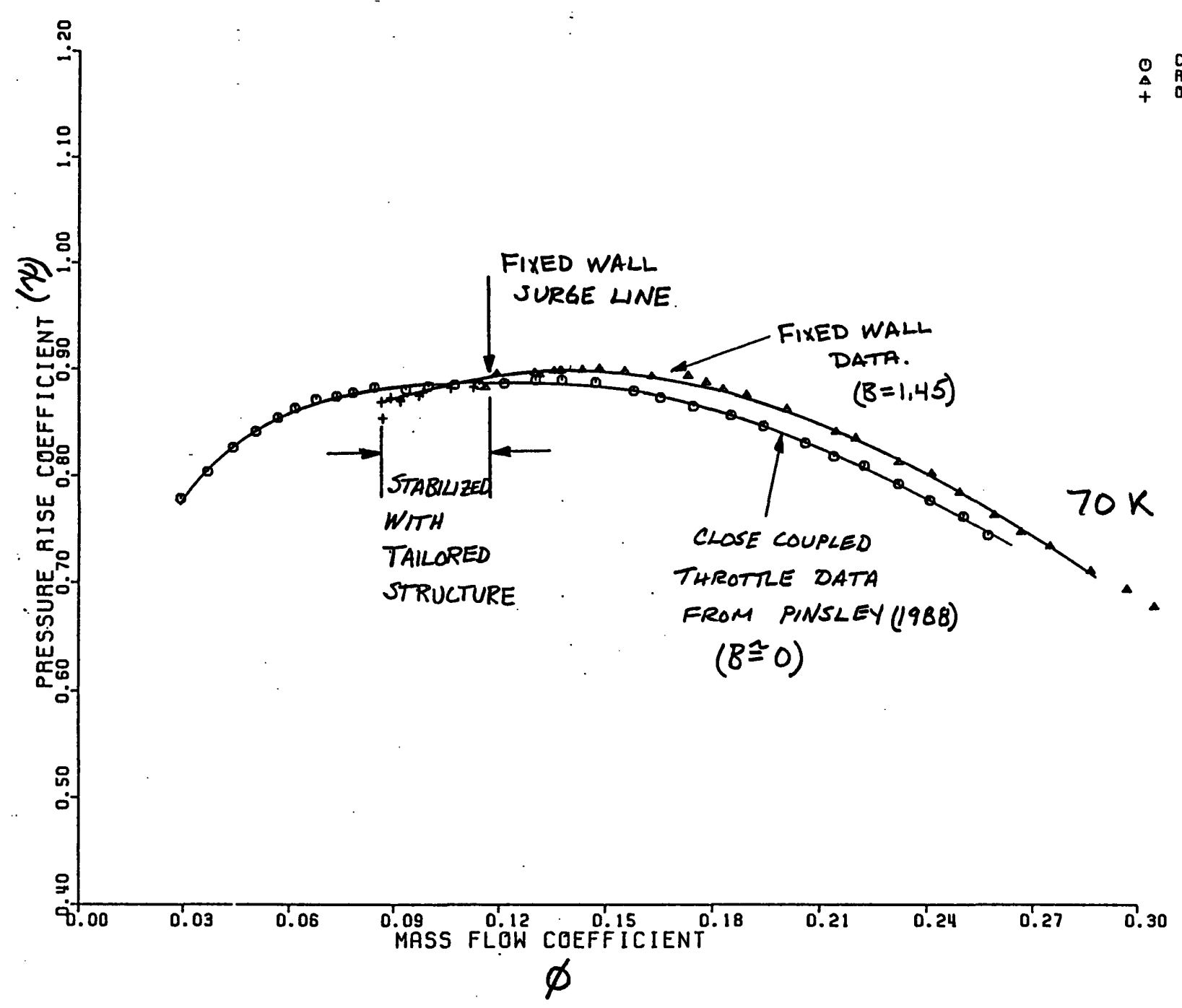

Figure 4.5: Comparison of Close Coupled and Structurally Stabilized Speedline ( $70 \mathrm{~K}$ ) 


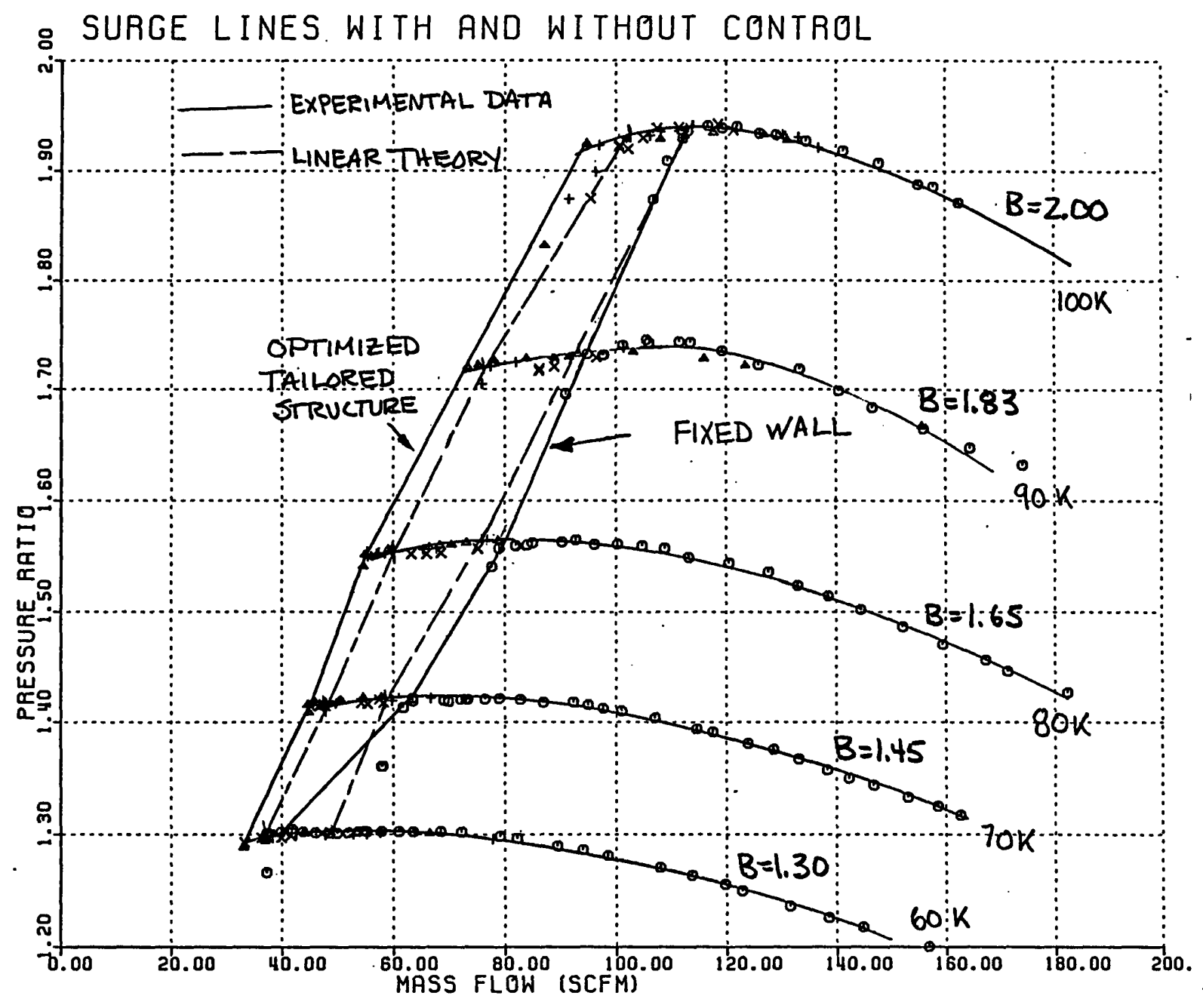

Figure 4.6: Predicted and Experimental Surgelines Fixed Wall and Optimized Systems 


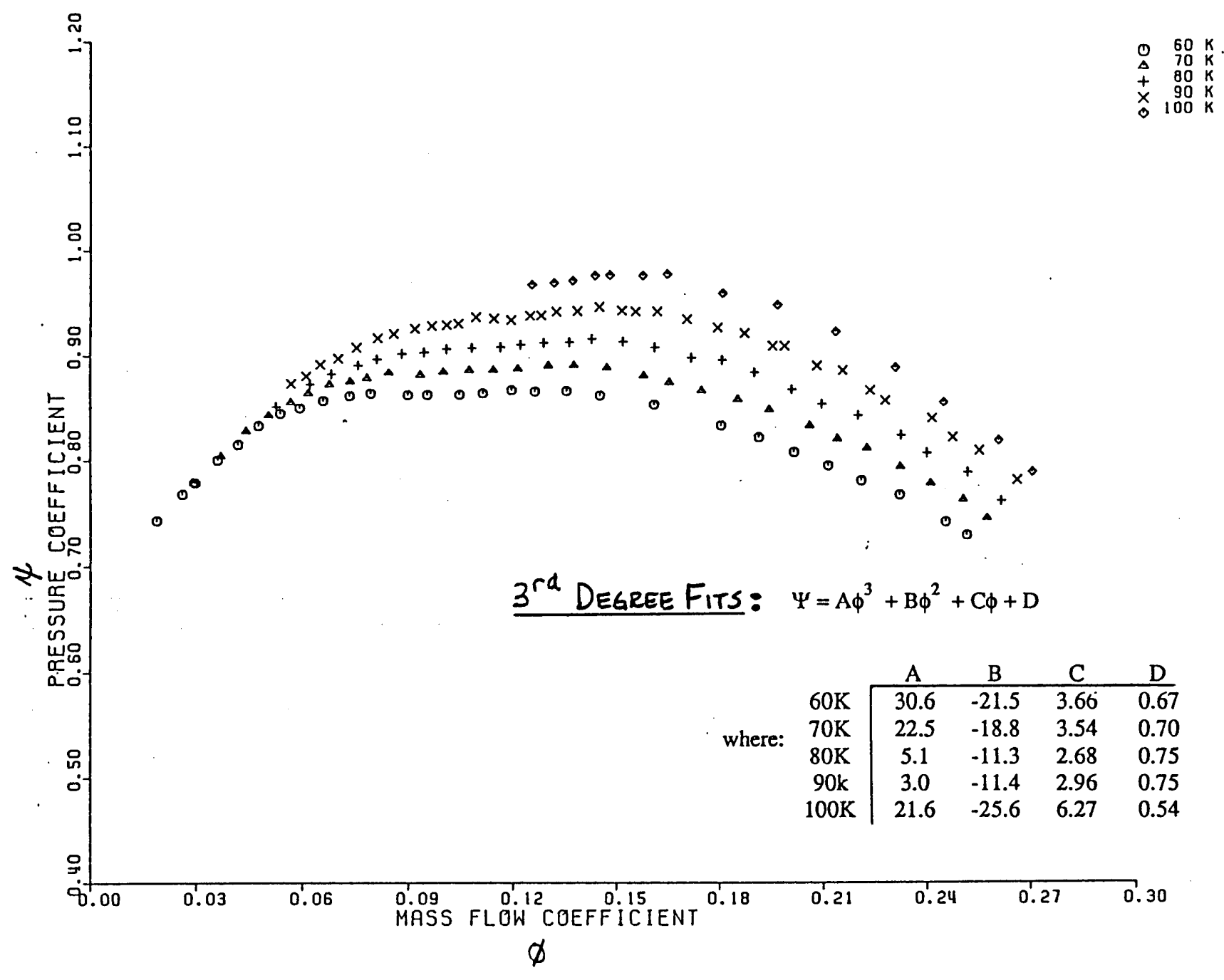

Figure 4.7: Close Coupled Speedlines for Holset H1D

(from Pinsley 1988) 


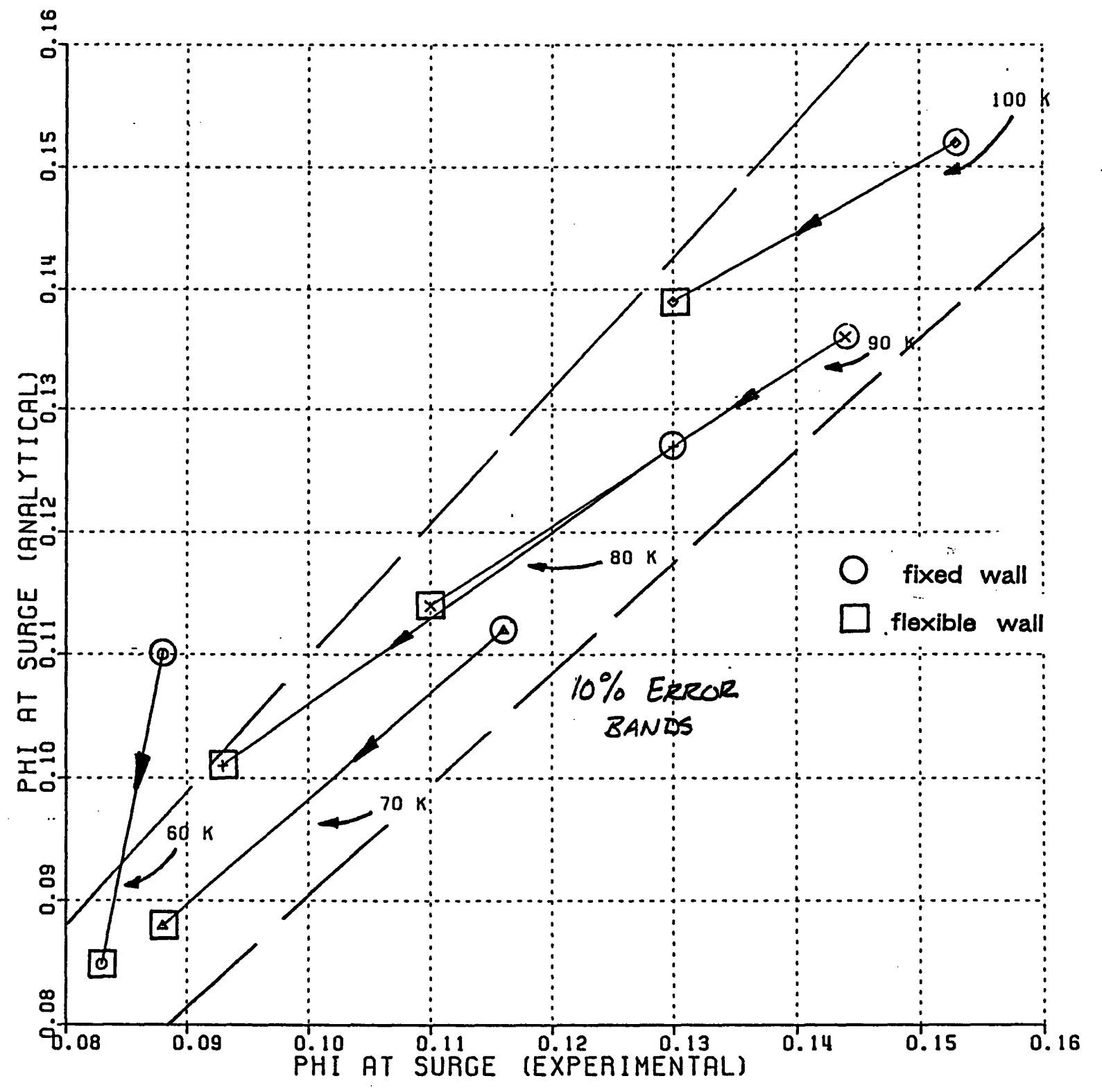

Figure 4.8: Predicted versus Experimental Mass Flow

Coefficients at Deep Surge

(Fixed Wall and Optimized Systems) 

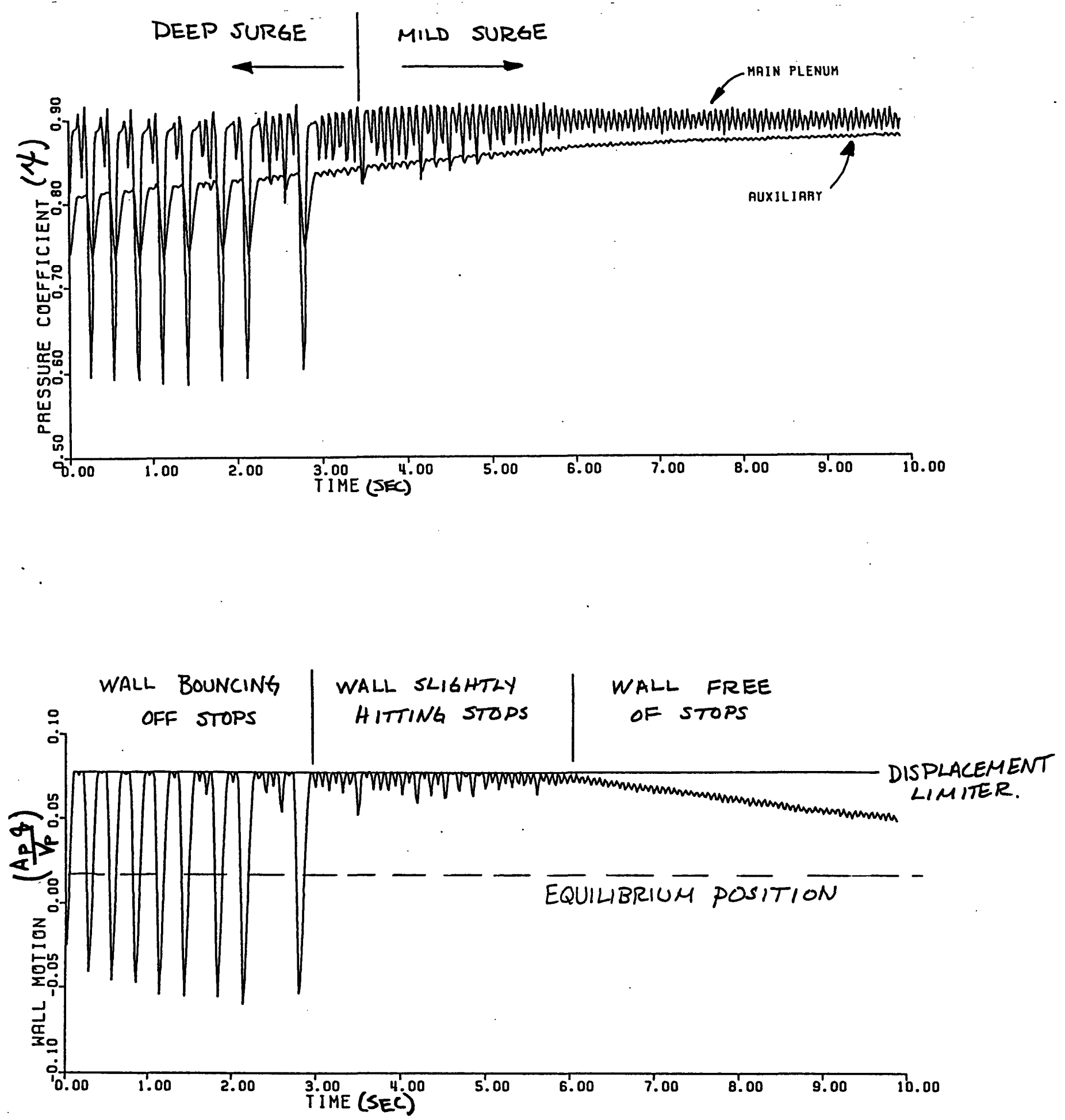

Figure 4.9: Suppression of Fixed Wall Deep Surge with

Flexible Wall 


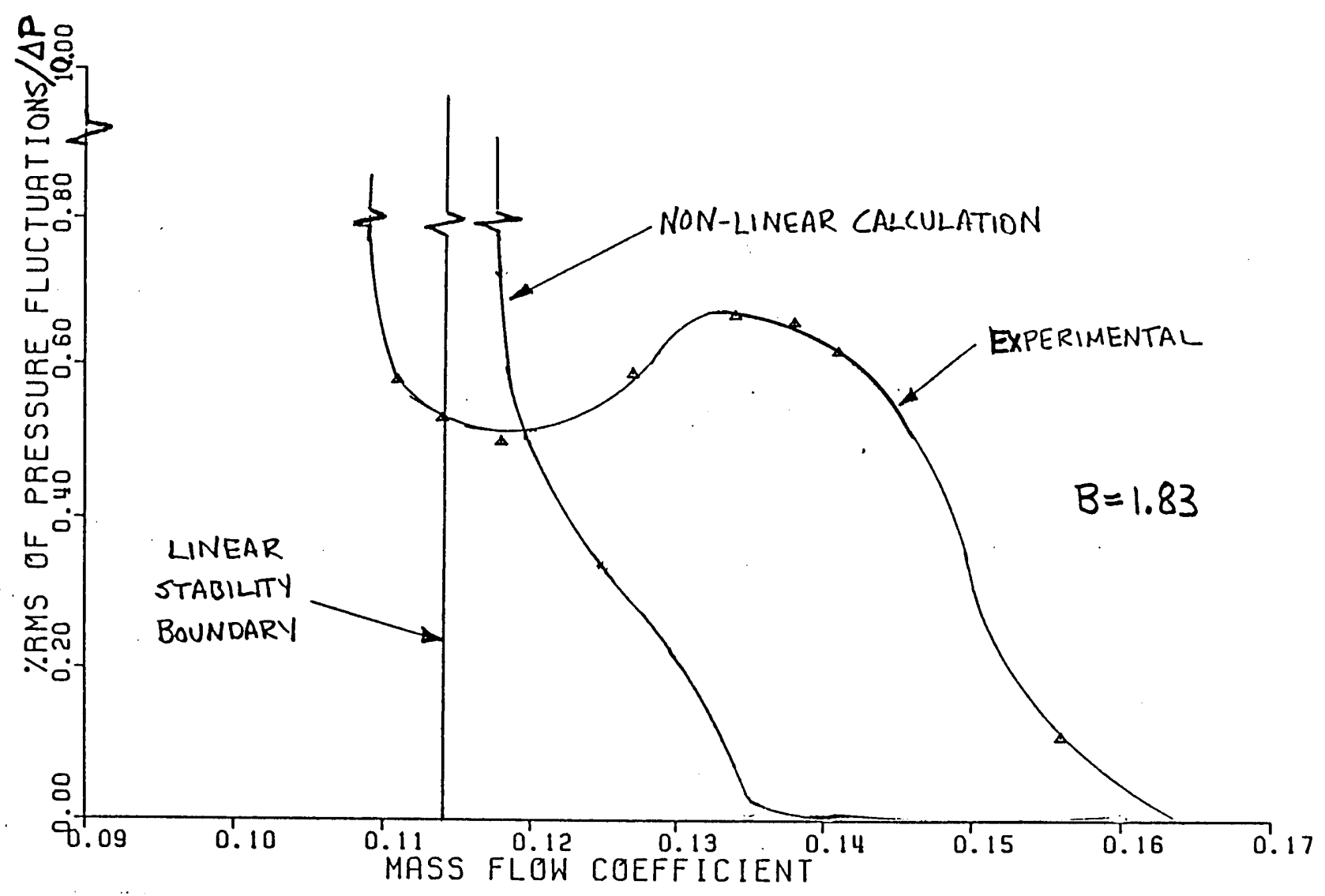

Figure 4.10: Amplitude of Plenum Pressure Fluctations Theory versus Experiment

$$
(\mathrm{B}=1.83)
$$




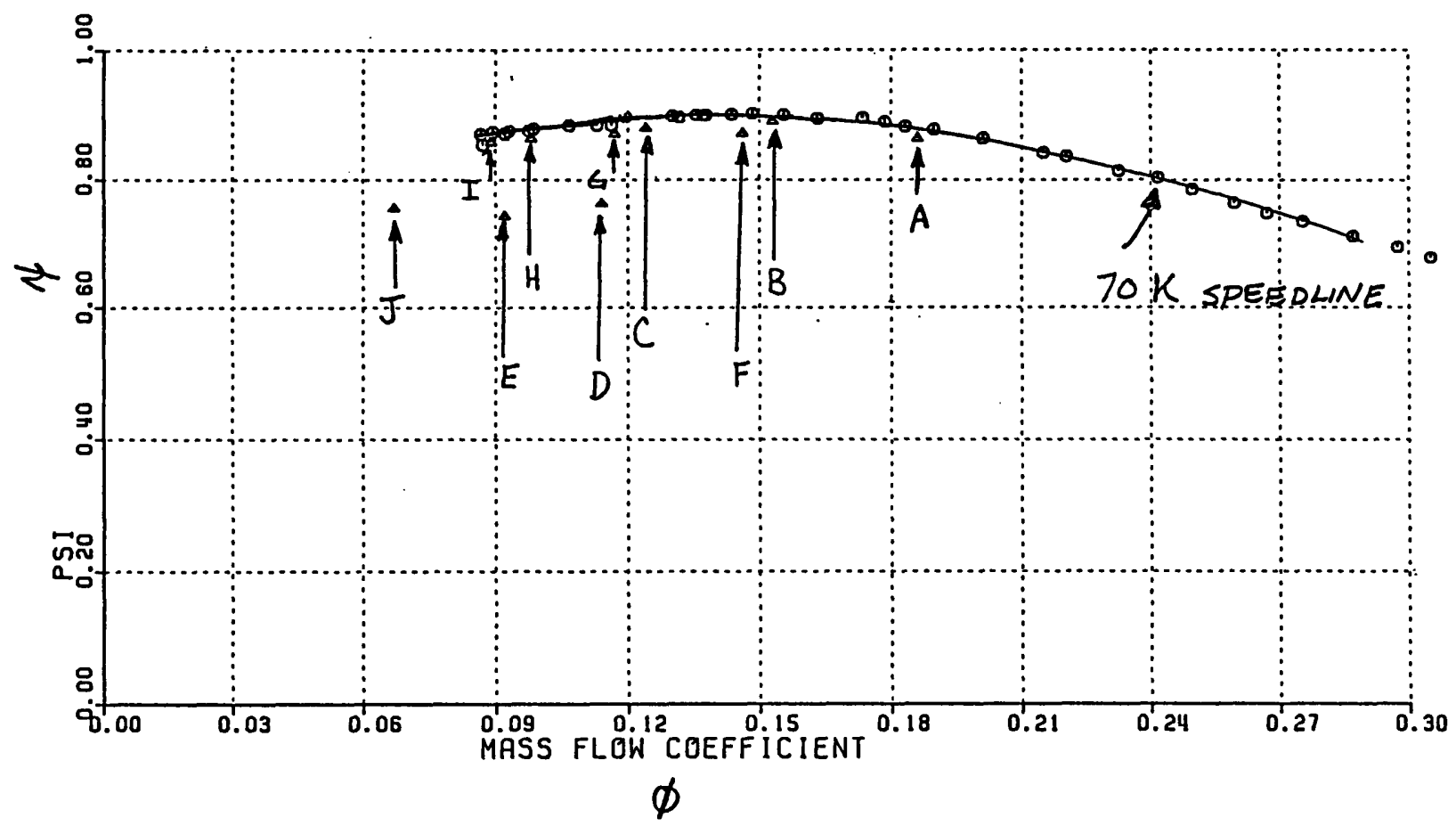

Figure 4.11: Steady Operating Points for Transient Data 
PT $A=.188$
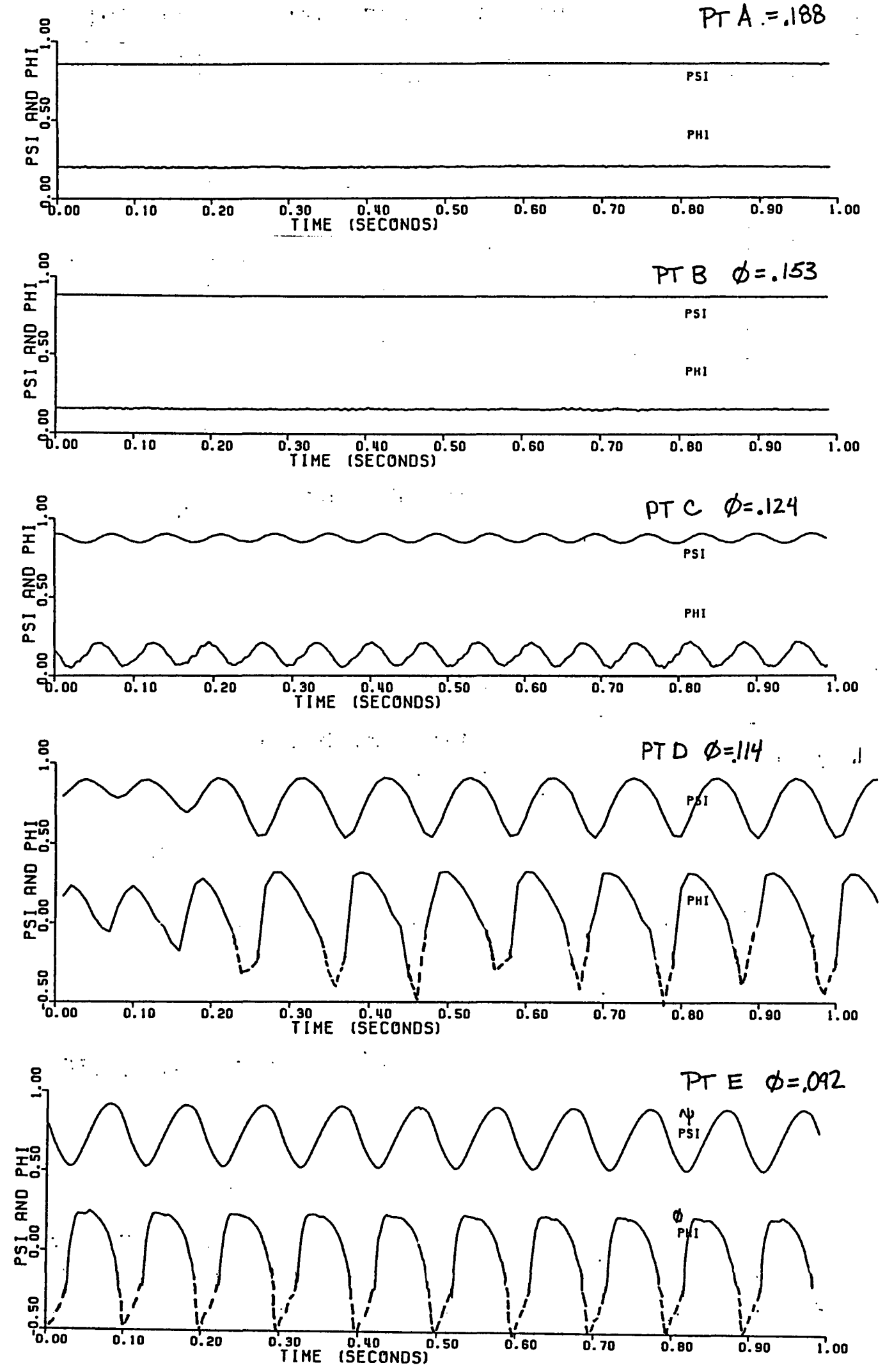

Figure 4.12: Fixed Wall Transient Behavior 
MILD SURGE
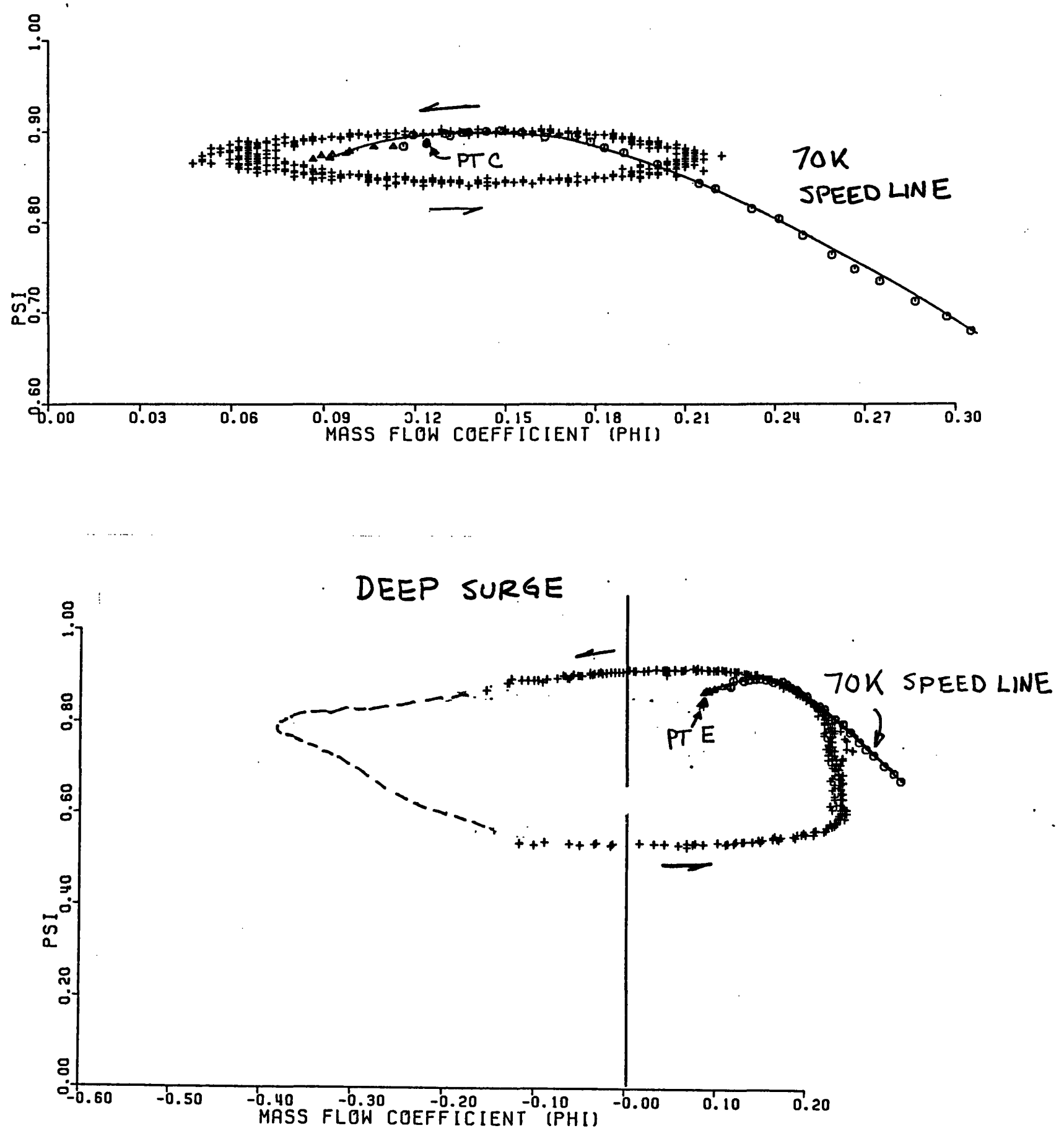

Figure 4.13: Surge Cycles for Fixed Wall System

(Points $\mathrm{C}$ and $\mathrm{E}$ ) 


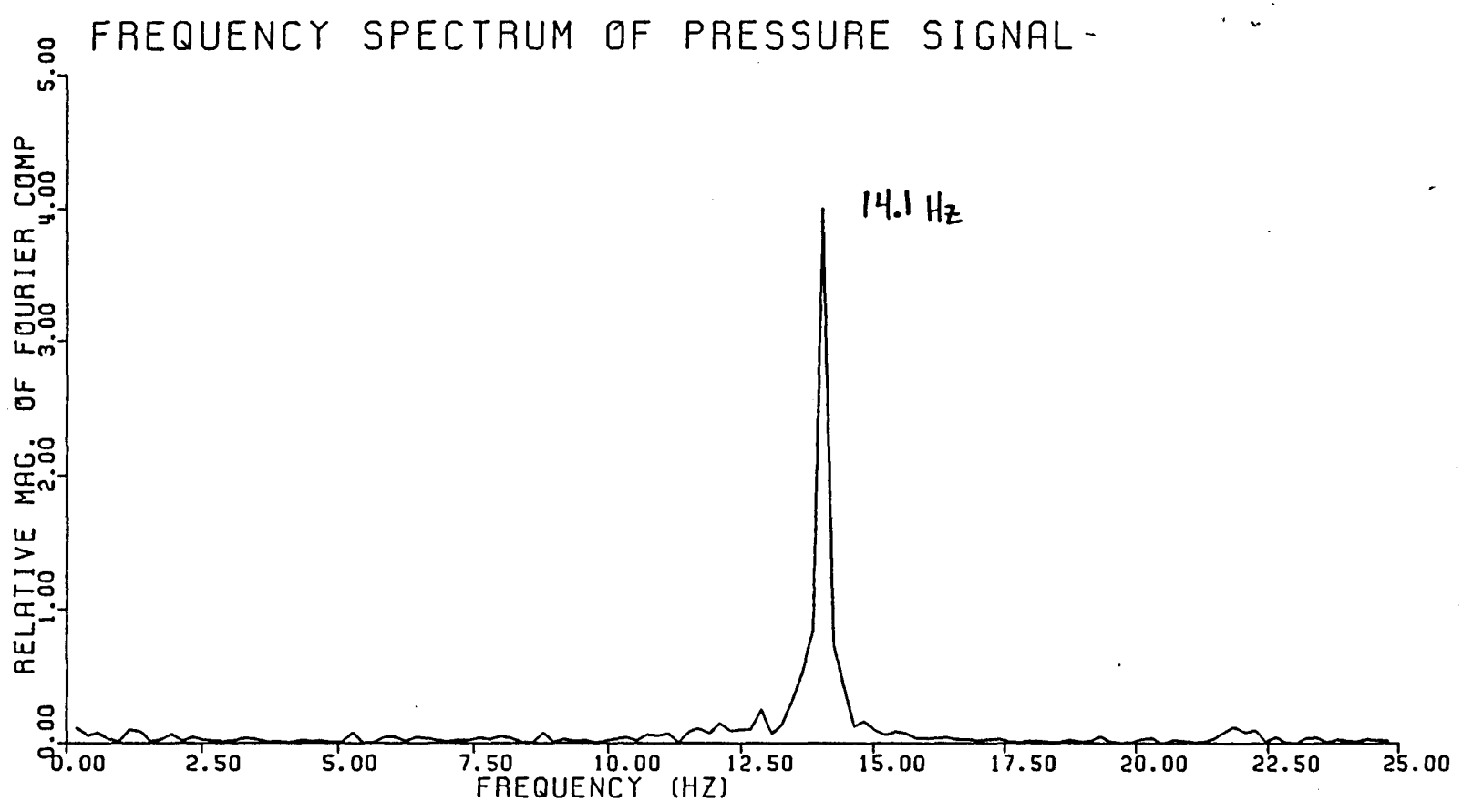

Figure 4.14: Frequency Spectrum of Fixed Wall Mild Surge Cycle $(B=1.45 ; \phi=.121)$ 

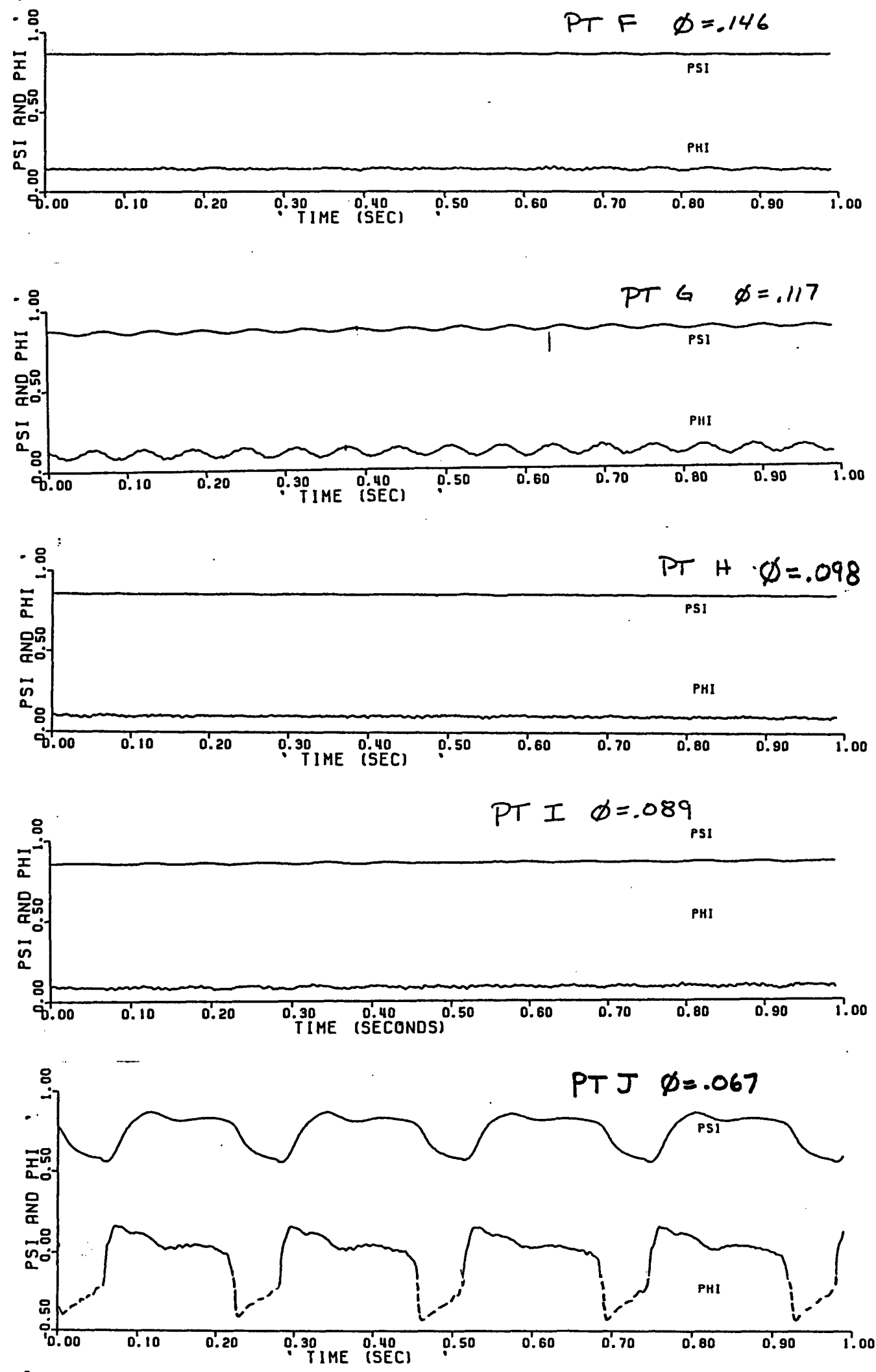

Figure 4.15A: Flexible Wall System Behavior

(Medium Damping Configuration: $B=1.45$ ) 

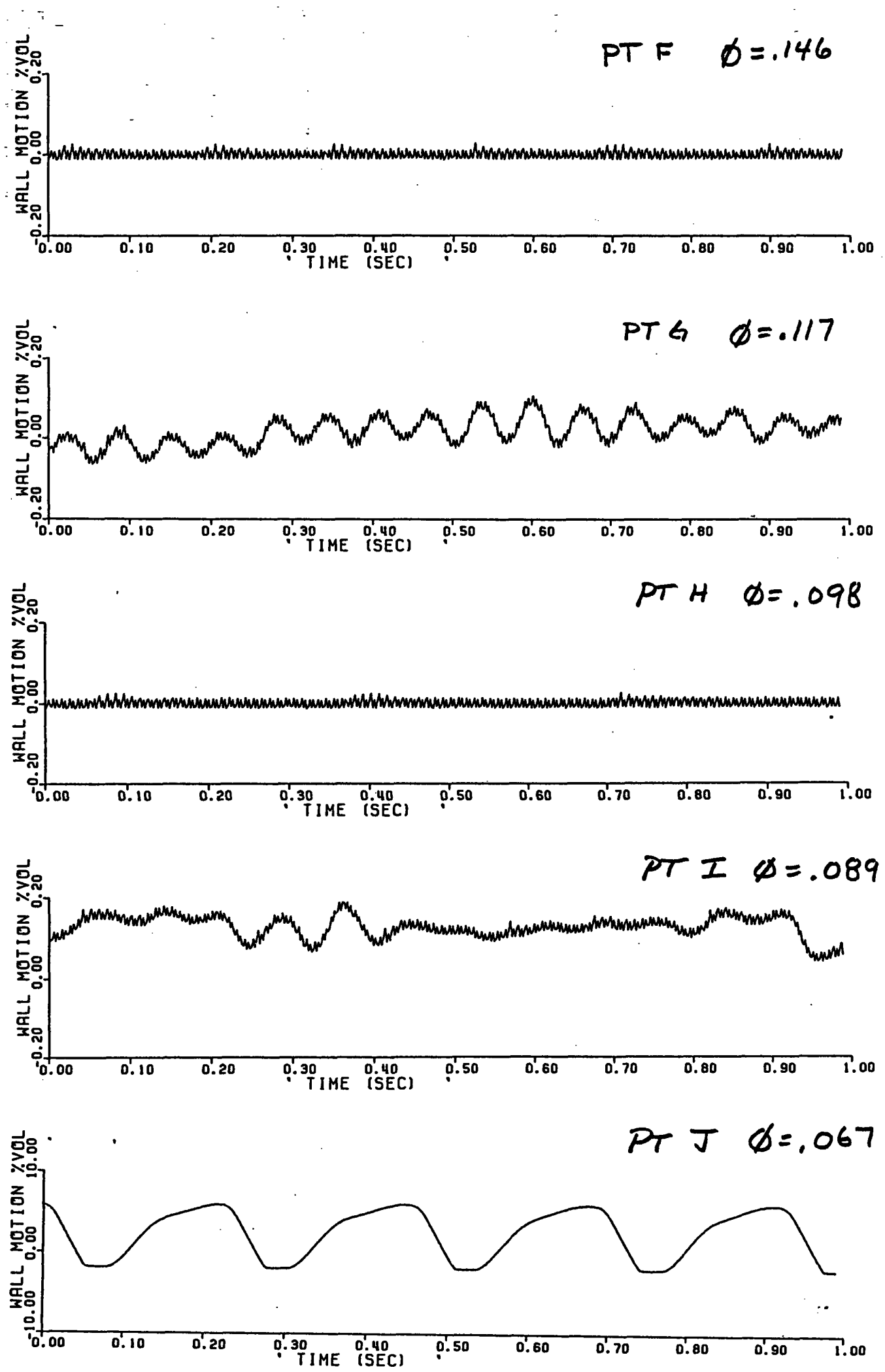

Figure 4.15B: Wall Motion for Points F - J 

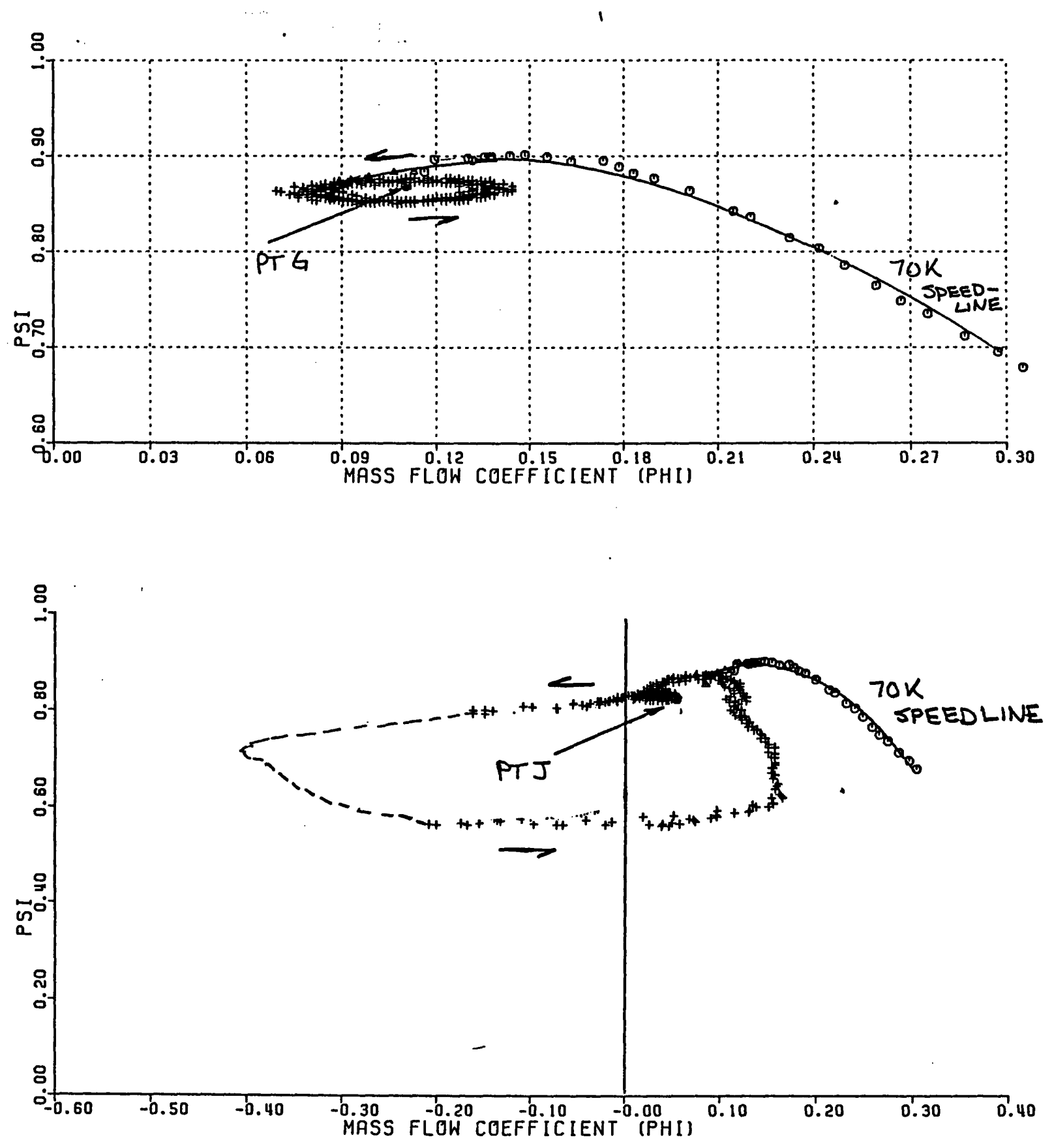

Figure 4.16: Surge Cycles for Flexible Wall System

(Points $\mathrm{G}$ and $\mathrm{J}$ ) 


\section{DISCLAIMER OF QUALITY}

Due to the condition of the original material, there are unavoidable flaws in this reproduction. We have made every effort possible to provide you with the best copy available. If you are dissatisfied with this product and find it unusable, please contact Document Services as soon as possible.

Thank you.

$$
\begin{aligned}
& \text { Page } 110 \text { is missing in the } \\
& \text { original document. }
\end{aligned}
$$



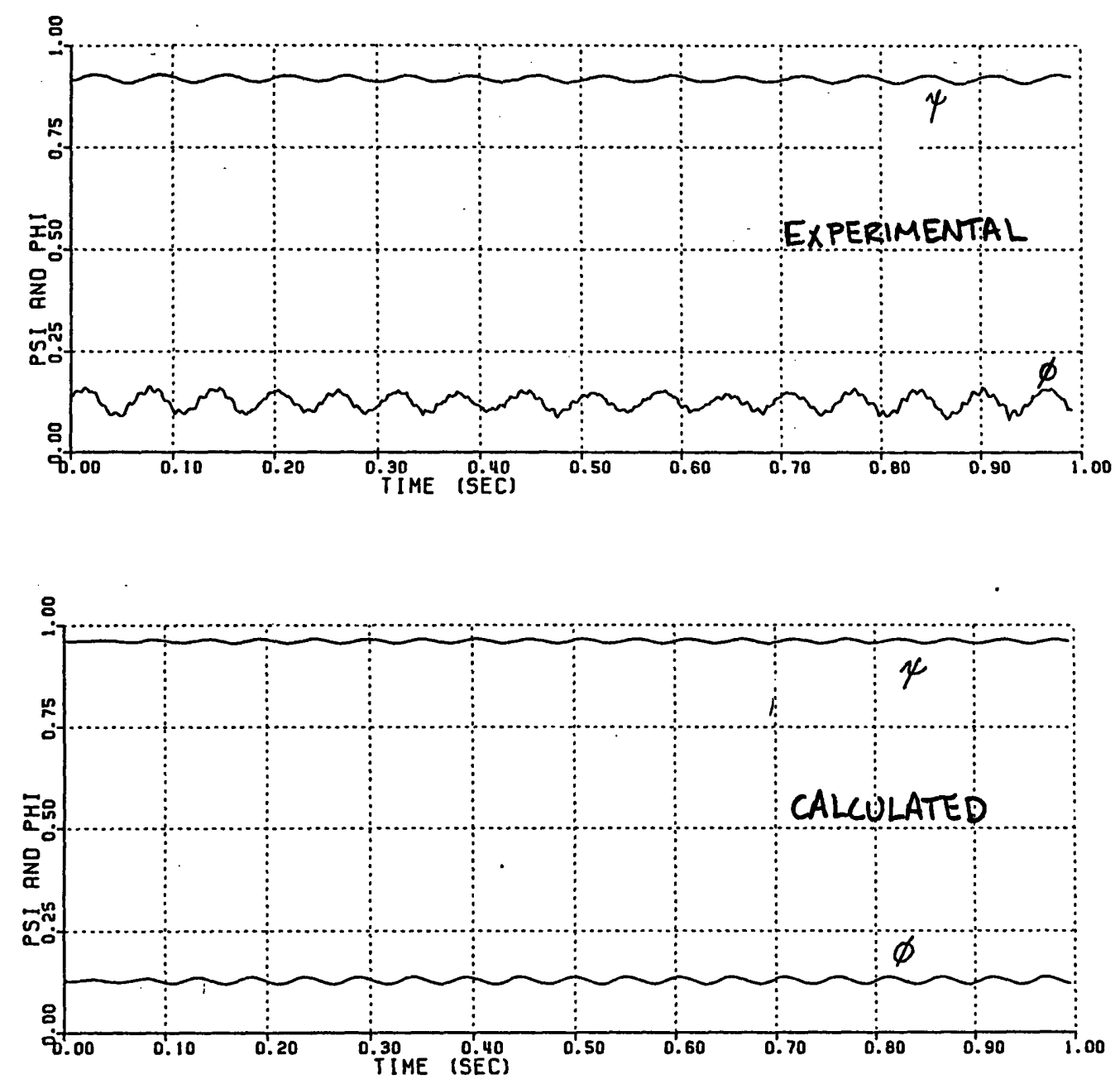

Figure 4.18A: Experimental and Calculated Mild Surge Cycles

$$
\text { ( } \mathrm{B}=1.83 ; \phi=.128 \text { ) }
$$

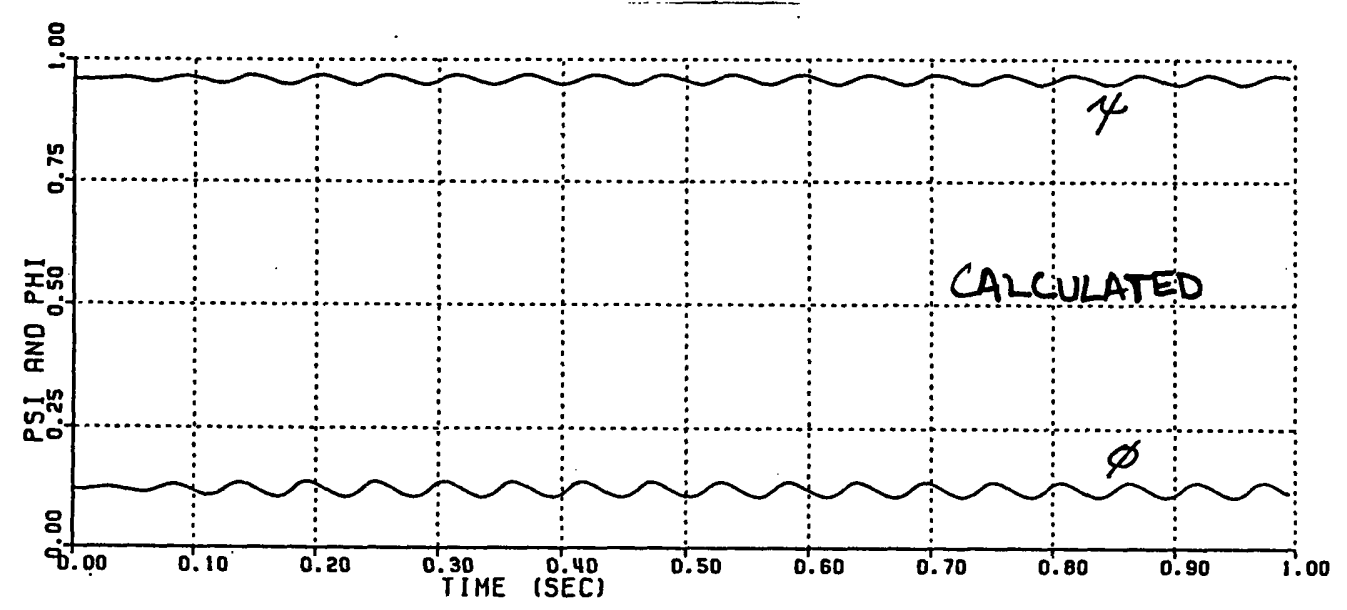

Figure 4.18B: Calculated System Behavior Matching Amplitude of Pressure Limit Cycle to Experimental Data

$$
\text { ( } \mathrm{B}=1.83 ; \phi=.124 \text { ) }
$$



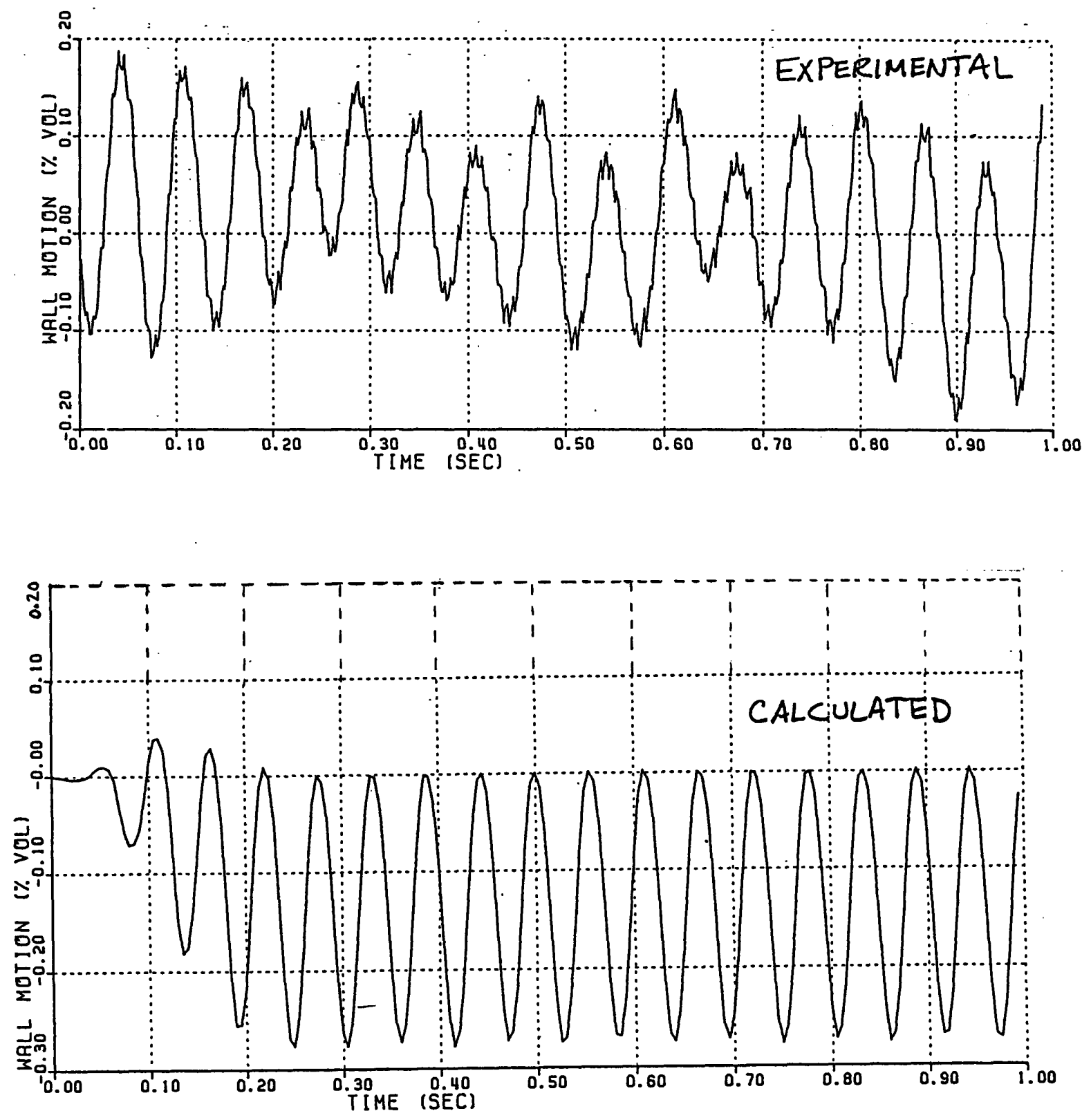

Figure 4.19: Comparison of Calculated versus Experimental Wall Motion Matching Amplitude of Pressure Limit Cycle

$$
(\mathrm{B}=1.83)
$$




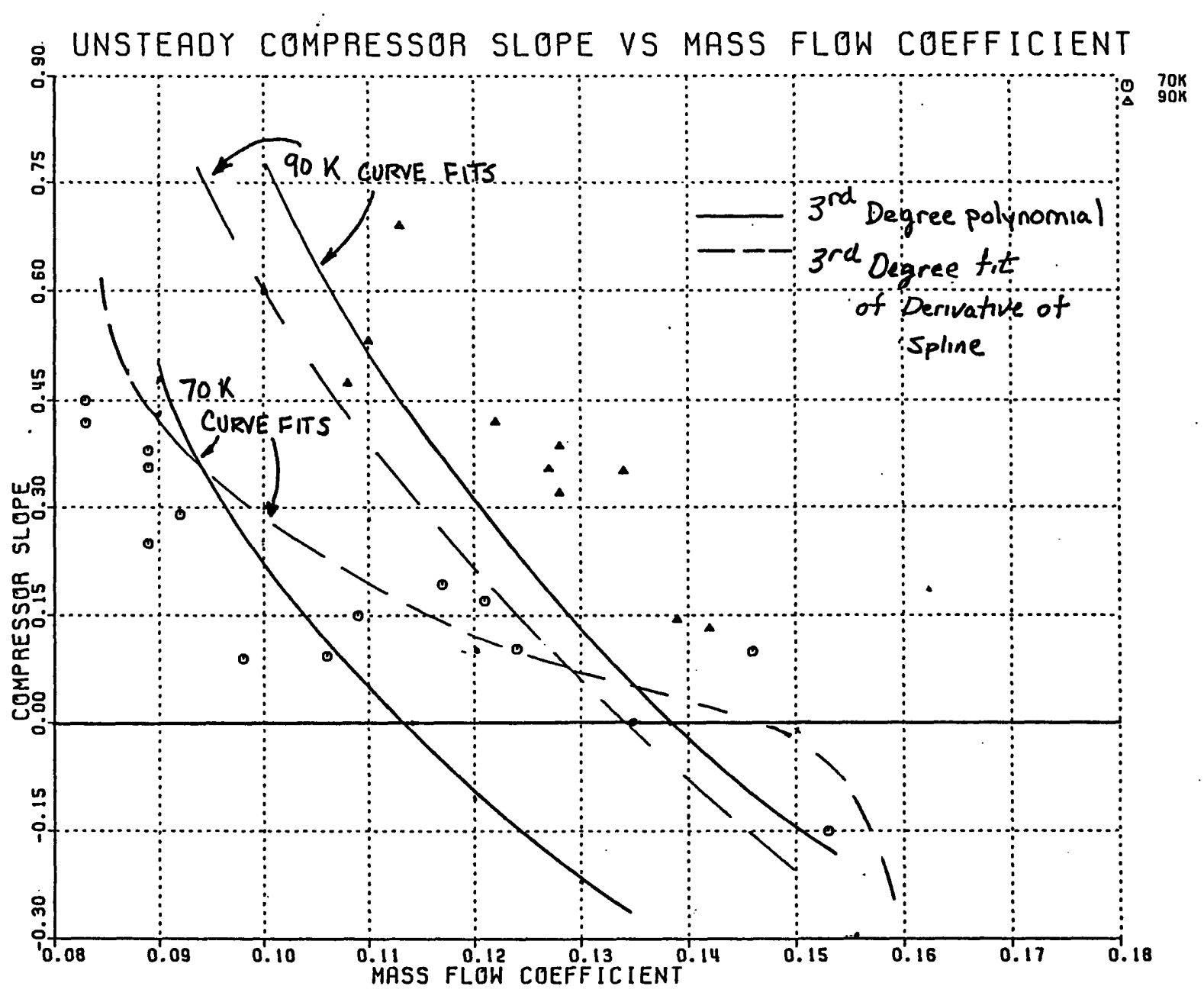

Figure 4.20: Measured Instantaneous Compressor Slope versus Phi (70 $\mathrm{K}$ and $90 \mathrm{~K}$ Speedlines) 

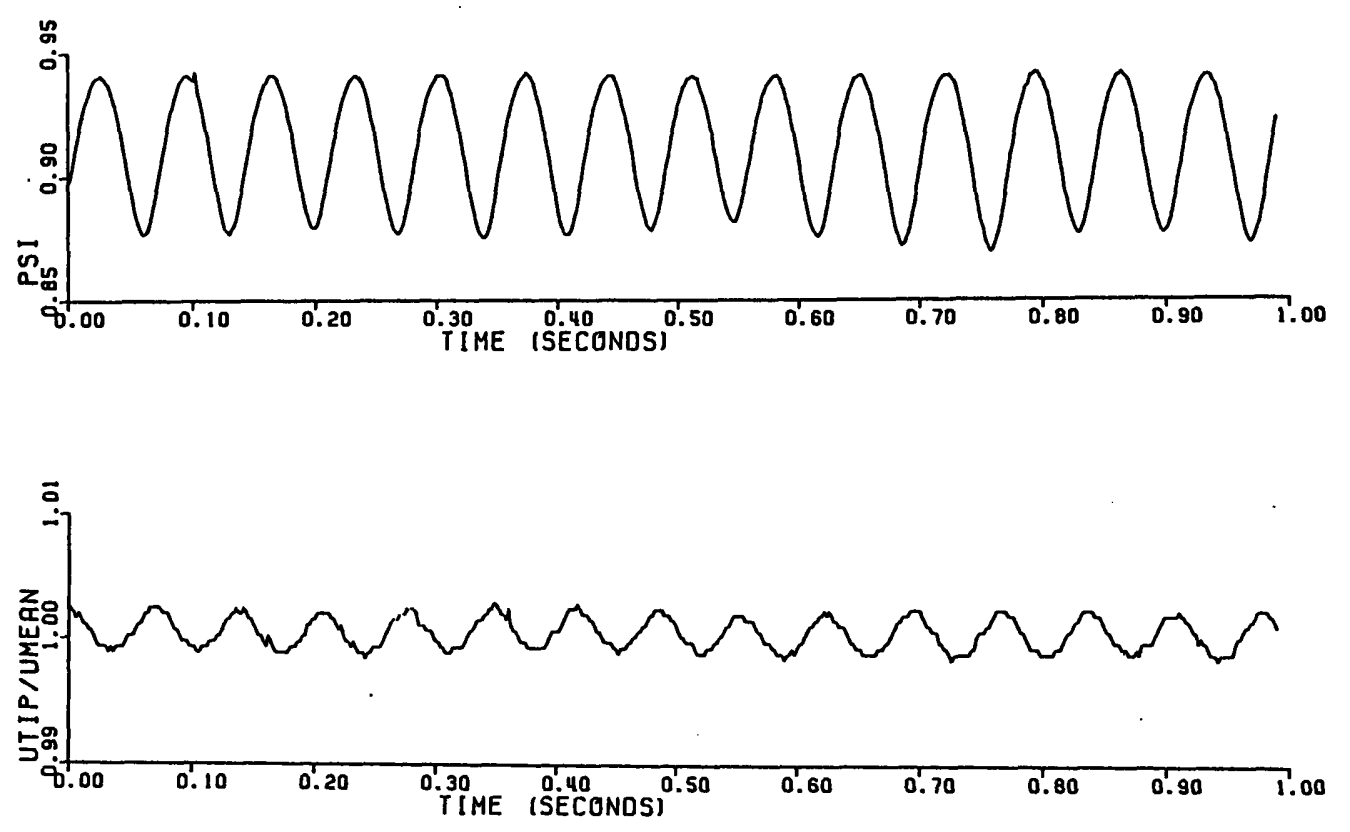

Figure 4.21A: Wheel Speed and Plenum Pressure Fluctations During Fixed Wall Mild Surge

$$
(\mathrm{B}=1.83 ; \phi=.142)
$$
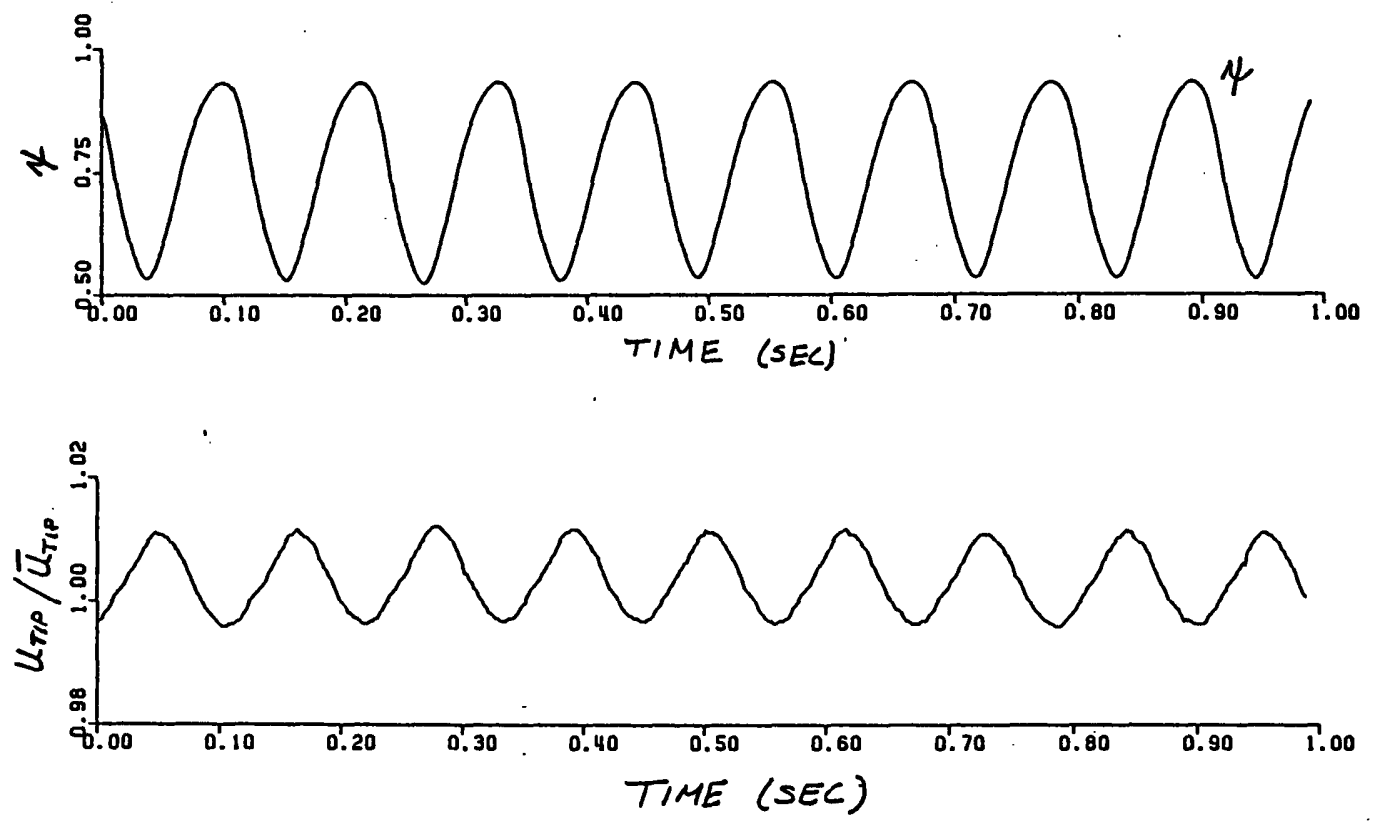

Figure 4.21B: Wheel Speed and Plenum Pressure Fluctuations During Fixed Wall Deep Surge

$$
\text { ( } \mathrm{B}=1.83 ; \phi=.105)
$$




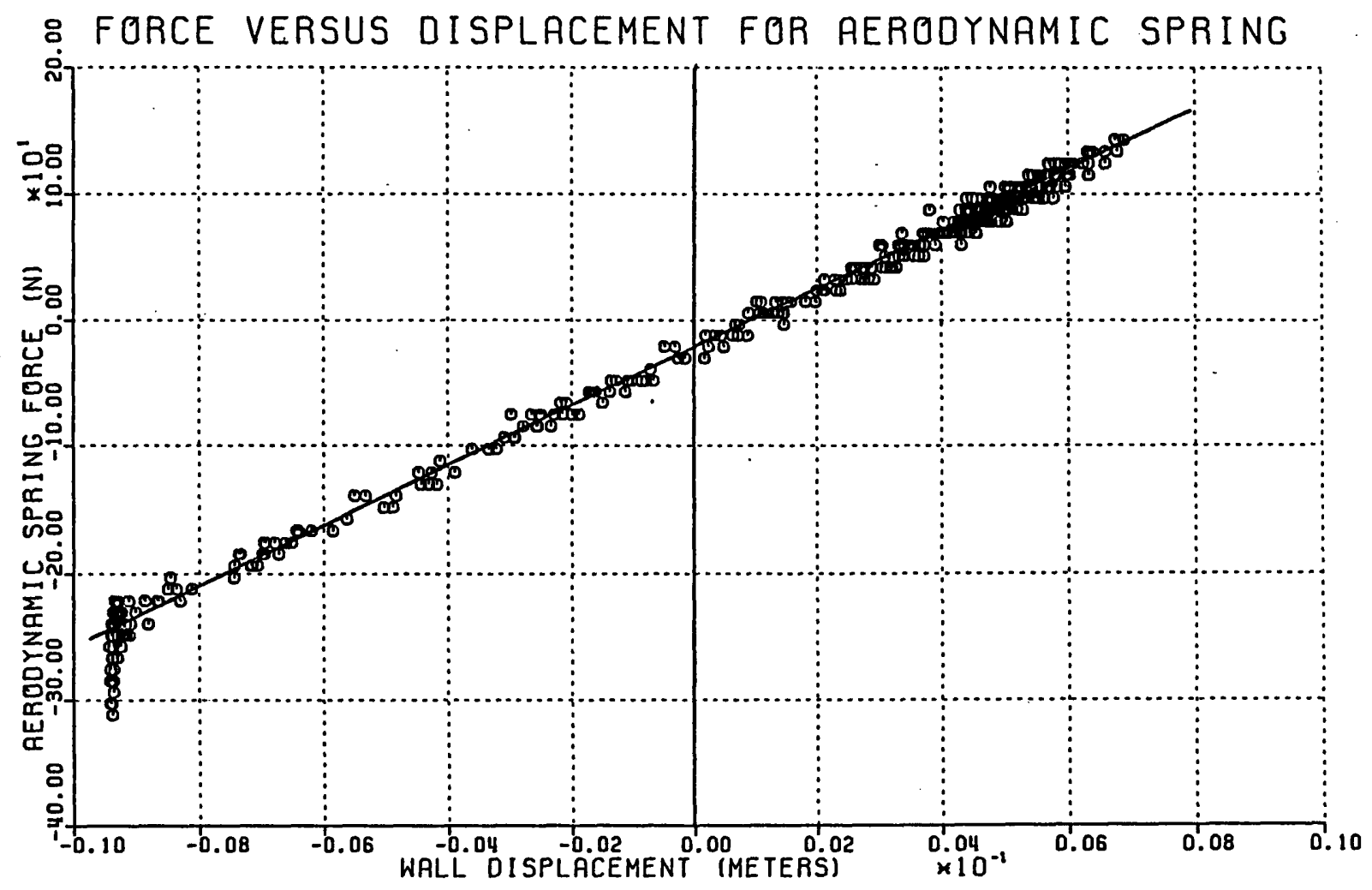

Figure 4.22: Aerodynamic Spring Force versus Displacement During Deep Surge 

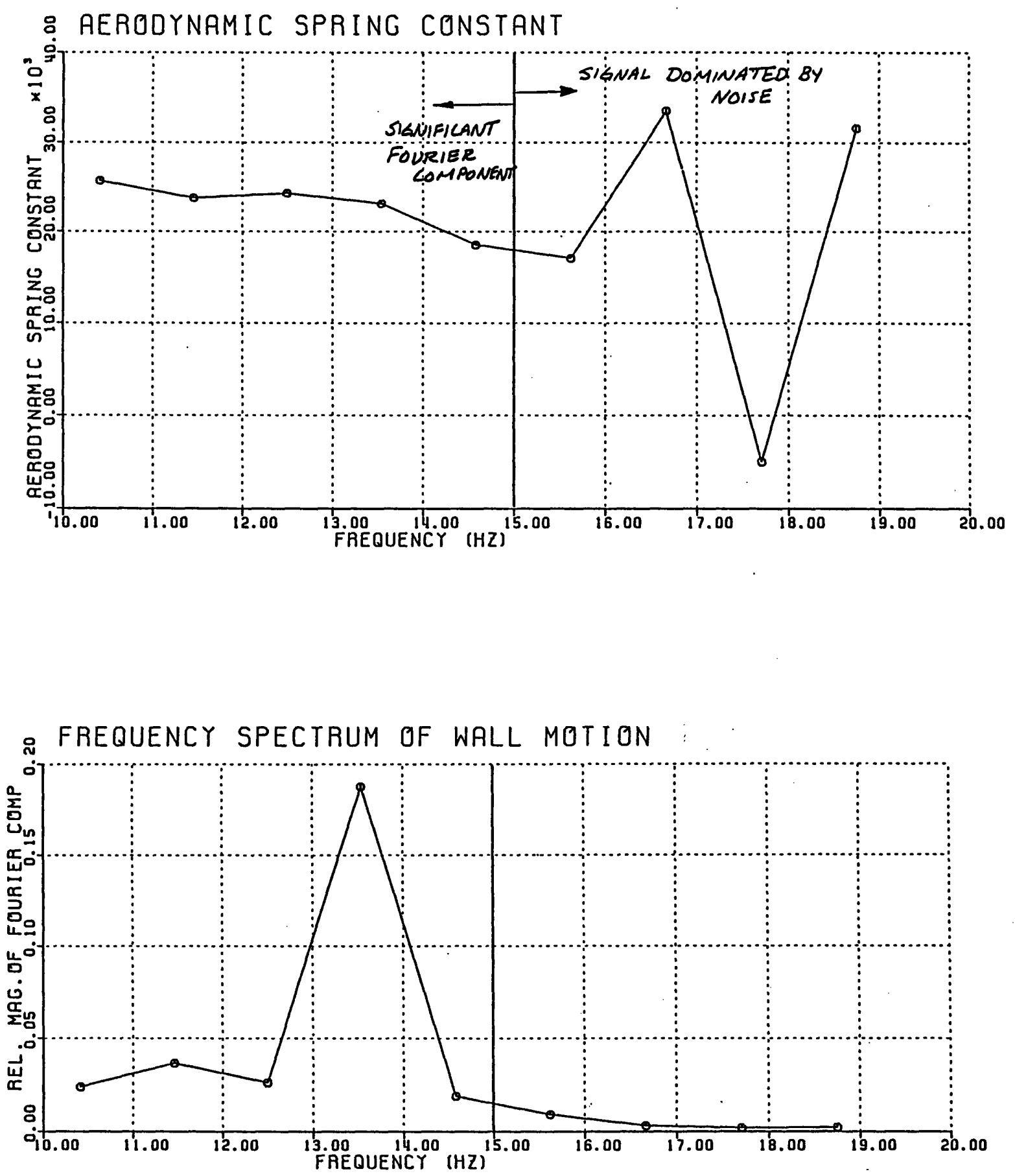

Figure 4.23: Aerodynamic Spring Constant Calculated in Frequency Domain 

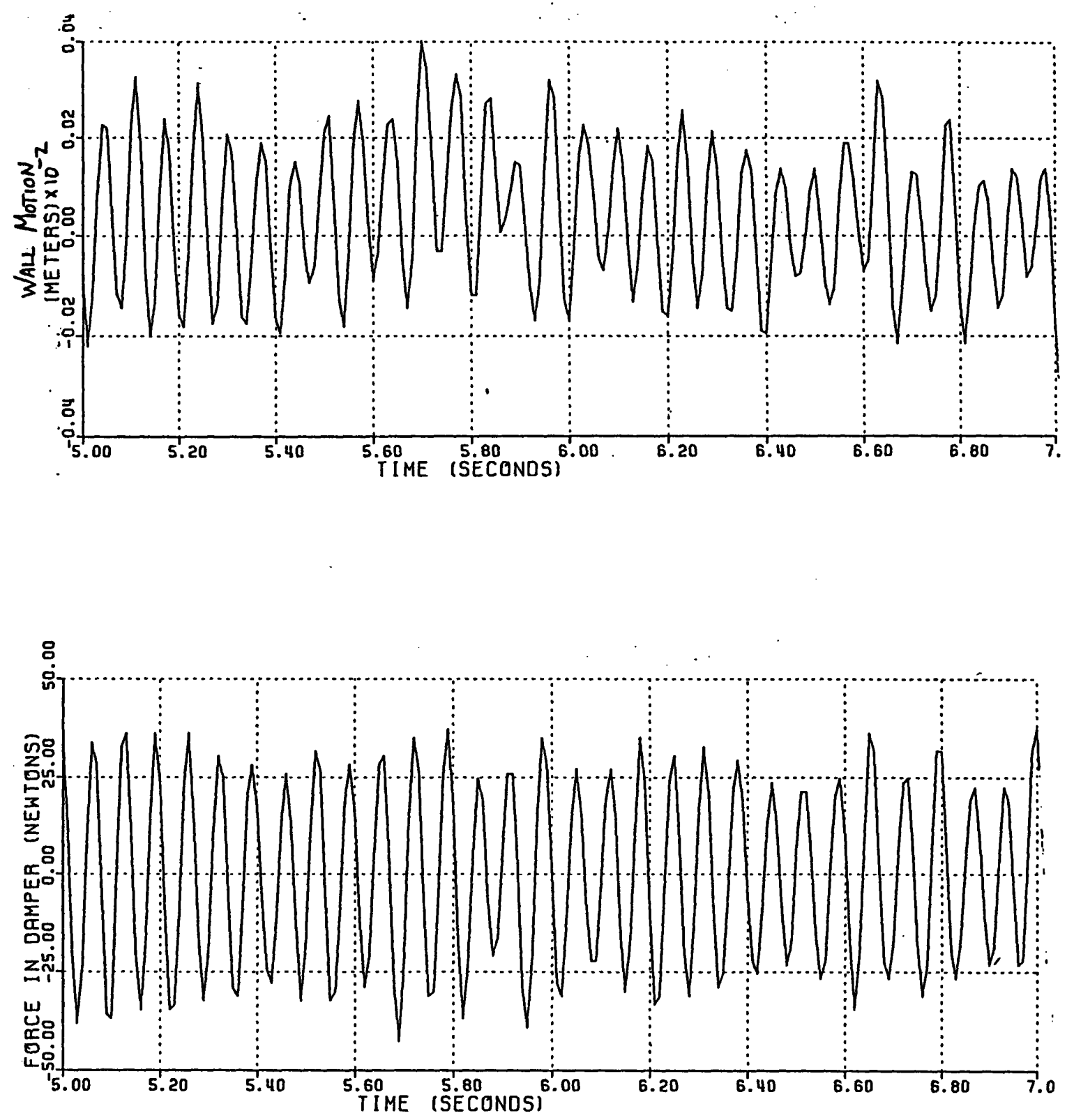

Figure 4.24: Wall Motion And Damper Force in Stabilized Region Medium Damping Configuration

$$
(\mathrm{B}=1.83 ; \phi=.131 \text { ) }
$$



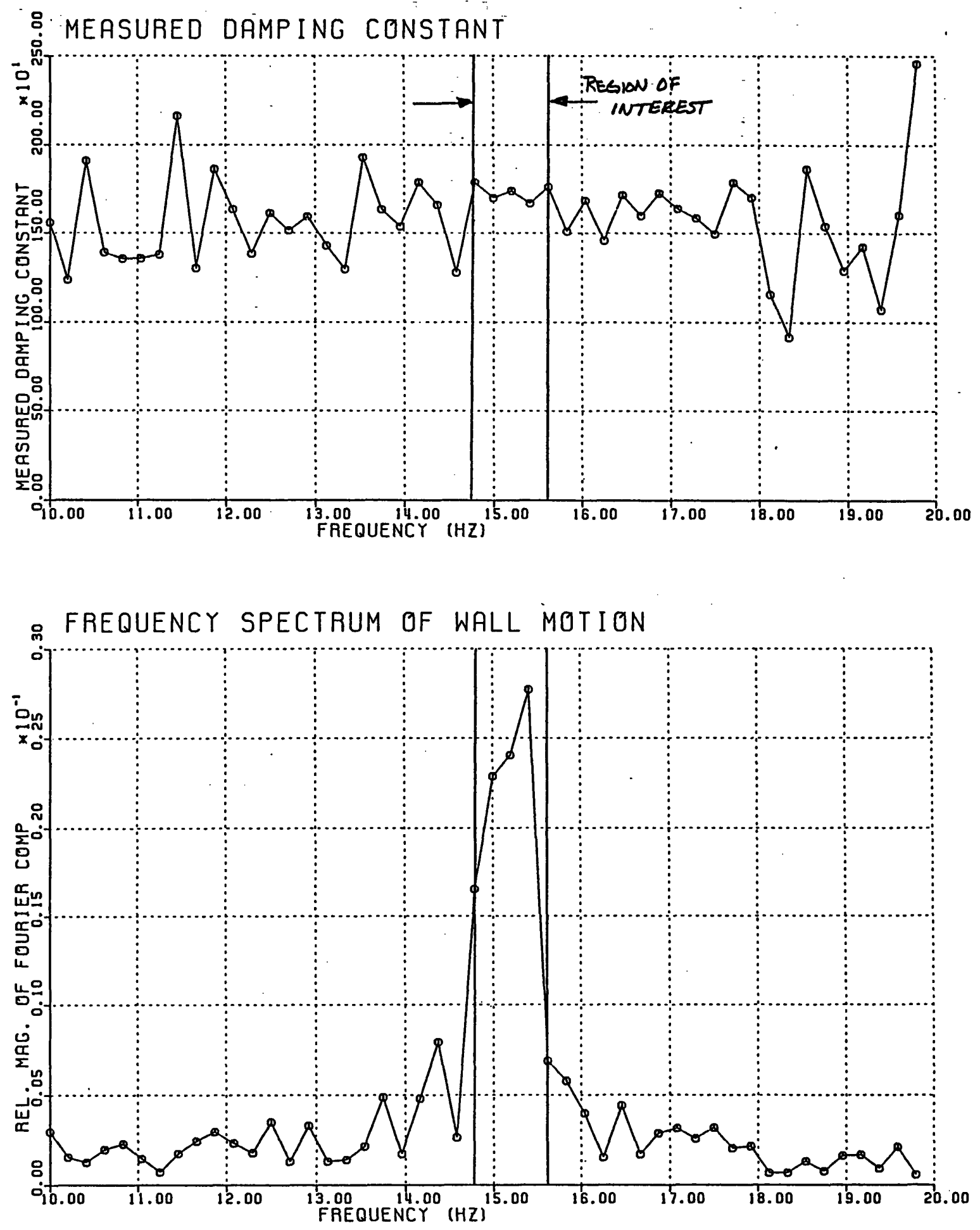

Figure 4.25: Damping Constant as Function of Frequency and Frequency Spectrum of Wall Motion

\author{
In Stabilized Region \\ Medium Damping Configuration
}

$$
\begin{gathered}
(B=1.83 ; \phi=.131) \\
118
\end{gathered}
$$




\section{CONCLUSIONS AND RECOMMENDATIONS}

\subsection{CONCLUSIONS FROM EXPERIMENT}

Dynamic control using tailored structure has been shown to be effective in suppressing centrifugal compressor surge. The use of a tailored plenum wall shifted the surge line to the left appproximately 25 to $30 \%$ in flow range over a significant portion of the compressor map, for B parameters ranging from 1.3 to 2.0. The effectiveness of surge suppression is a function of a set of non-dimensional parameters which govern the aeroelastic coupling of the wall to the compression system dynamics.

The present scheme was found to be quite robust, suppressing surge over a wide range of operating conditions and B-parameters with no adjustment to the parameters. The flexible wall was also demonstrated to be capable of suppressing an existing, (highly non-linear), surge cycle. In the stabilized region of the compressor map, the flexible wall supressed surge with no steady state performance penalty.

The amount of control action (wall motion) required is a function of the non-linearities in the wall dynamics, such as Coulomb friction and leakage. For the optimized configuration investigated in this research, the nominal wall motion in the stablized region was roughly $0.1 \%$ of plenum volume, oscillating at frequencies near the Helmholtz frequency. Pressure fluctuations in the stabilized region were on the order of $0.5 \%$ of the mean pressure rise of the compressor.

Unsteady, time-resolved data was used to verify some of the major assumptions in the modelling of the compression system. The compressor transfer function showed that the quasi-steady compressor characteristic was a reasonable representation of the instantaneous compressor slope. The compressor slope measurement also showed that the improvement in surge margin is due to modification of the system dynamics, rather than a result of modifying the compressor characteristic by altering the local details of the flow in the compressor only. Wheel speed variations recorded during mild surge cycles supported predictions that wheel speed variation for this rig would not significantly effect system stability. 
The unsteady data was also used to characterize the performance of components in the flexible wall apparatus. Both the aerodynamic spring and the viscous dashpot were shown to behave linearly as modelled.

\subsection{CONCLUSIONS FROM ANALYSIS}

The results of the experimental investigations, confirmed that the Helmholtz resonator model of the compression system was an accurate model of the system dynamics, with and without the flexible wall. The linearized form of the model accurately predicted the interaction of the wall and the compression system dynamics through the non-dimensional structural control parameters.

The physical mechanism responsible for the surge suppression with the flexible wall was shown to be the direct unsteady energy dissipation in the wall motion. The increase in stable flow range was not a result of modification of the system dynamics to cause the throttle to dissipate more unsteady energy.

The maximum stable compressor slope on the non-dimensional pressure rise versus mass flow characteristic was shown to be a better measure of the effectivness of a control scheme than the minimum stable mass flow coefficient. The steady state mass flow coefficient at instability has little direct effect on the stability of the compression system with the flexible wall, but rather acts only through the relation between mass flow and the compressor slope, via the characteristic.

Non-linear solution of the system equations showed the existence of small amplitude limit cycles in the stabilized region in close agreement with measurement. These limit cycles were determined to be a result of Coulomb friction, leakage, and non-linearities in the throttle and compressor characteristics. The Coulomb friction and leakage effects are the dominant cause of the small amplitude limit cycles in the stabilized regions of the compressor map. 


\subsection{RECOMMENDATIONS FOR FUTURE WORK}

The results in this thesis are encouraging in that they demonstrate clearly that the flexible plenum wall control strategy is effective in suppressing surge. In addition the lumped parameter model accurately predicts the behavior of the compression system with the flexible plenum. The success of the model in predicting the behavior of the experimental rig indicates that the other control strategies developed in the appendices will also perform as predicted. Therefore, the aerodynamic and structural dynamic feedback control strategies developed in the Appendices may warrant further investigation.

Additional work is also needed in developing more application oriented mechanical devices to implement the passive plenum volume control strategy to various types of turbomachinery. The analyses developed in the present work can be applied to any configuration that exhibits the dynamic characteristics of the flexible plenum wall.

The feasibility of aeroelastic control of multi-dimensional instabilities (rotating stall) using tailored structure is a natural extension of the thesis and should be explored. Control of rotating stall is critical before the benefits of surge suppression can be fully realized in axial flow compressors. 


\section{REFERENCES}

1) Fink, D.A., "Surge Dynamics and Unsteady Flow Phenomena in Centrifugal Compressors", Ph.D Thesis, Department of Aeronautics and Astronautics, MIT, May 1988.

2) Greitzer, E.M., "Surge and Rotating Stall in Axial Flow Compressors Part II: Experimental Results and Comparison with Theory", Journal of Engineering for Power, Transactions of ASME, Vol. 98, April 1976, pp.109-217.

3) Epstein, A.H., Ffowcs Williams, J.E., and Greitzer, E.M., "Active Suppression of Compressor Instabilities", AIAA-86-1994, AIAA 10th Aeracoustics Conference, July, 1986.

4) Emmons, H.W., Pearson, C.E., and Grant, H.P., "Compressor Surge and Stall Propagation", ASME Transactions, Vol.77, April 1955, pp. 455-469.

5) Greitzer, E.M.,"Surge and Rotating Stall in Axial Flow Compressors Part I: Theoretical Compression System Model", ASME 75-GT-9, Gas Turbine Conference, March 1975.

6) Greitzer, E.M., " The Stability of Pumping Systems -- The 1980 Freeman Scholar Lecture", Journal of Fluids Engineering, Vol. 103, June 1981, pp.193-242.

7) Stenning, A.H., "Rotating Stall and Surge", Journal of Fluids Engineering, Transactions of the ASME, Vol. 102, March 1980, pp.14-20.

8) Huang, X., "Active Control of Aerodynamic Instabilities", Ph.D. Thesis, Department of Engineering, University of Cambridge, July 1988.

9) Pinsely, J.E., "Active Control of Centrifugal Compressor Surge", M.S. Thesis, Department of Aeronautics and Astronautics, MIT, October 1988.

10) Chen, G.T., "Active Contol of Turbomachinery Instabilities -- Initial Calculation and Results", M.S. Thesis, Department of Aeronautics and Astronautics, MIT, August 1987.

11) Dean, R.C., Jr. and Young, L.R.,"The Time Domain of Centrifugal Compressor and Pump Stability and Surge", Journal of Fluids Engineering, Transactions of the ASME, March 1971, pp.53-63.

12) Halfman, R.L.,Dynamics_Addison-Wesley Publishing Company, Inc., Reading , Massachusetts, 1962.

13) Meirovitch, L, Elements of Vibration Analysis, McGraw-Hill Book Company, New York, 1986.

14) White, F.M., Fluid Mechanics, McGraw-Hill Book Company, New York, 1979. 
15) Ower, E. and Pankhurst, R.C., The Measurement of Air Flow, Pergamon Press Ltd., Oxford, 1966.

16) Hill, P.G., Peterson, C.R.,Mechanics and Thermodynamics of Propulsion, Addison-Welsey Publishing Company, Reading, Massachusetts, 1970. 


\section{APPENDIX A}

\section{Derivation of the Equations of Motion for the Flexible Plenum Wall System}

Consider the flexible plenum wall compression system shown below:

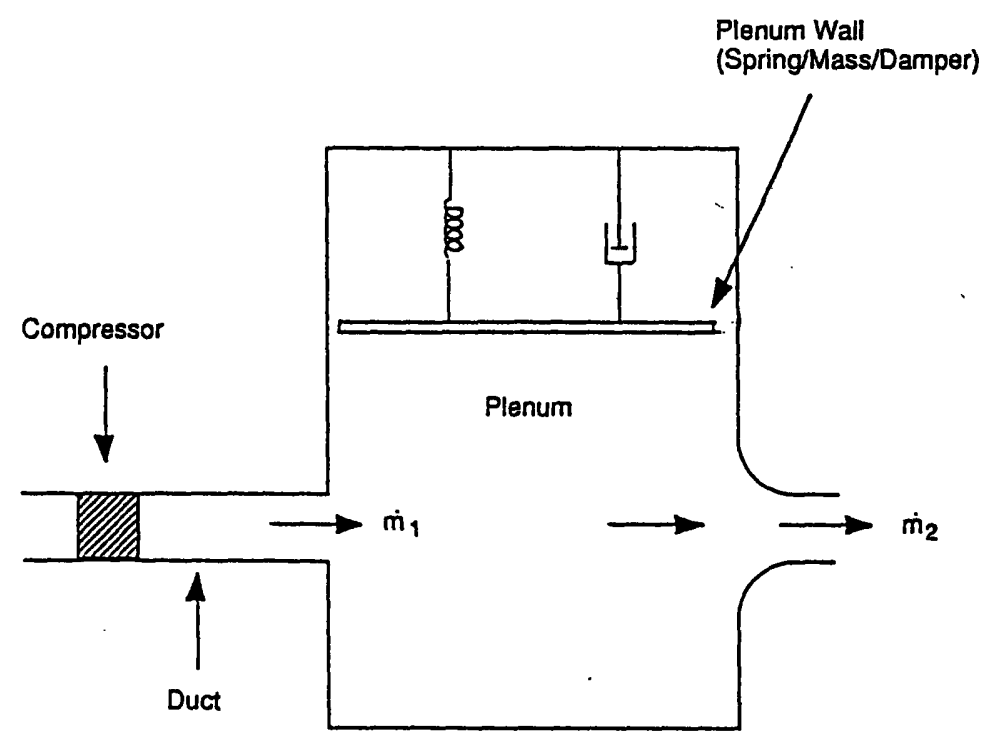

In deriving the equations of motion for the system, the follow basic assumptions were made.

1) Flow in the inlet ducting is 1-D, incompressible, inviscid, and unsteady

2) Plenum pressure is spatially uniform

3) Fluid in the plenum behaves isentropically

4) Fluid inertia in the throttle is negligible

5) Compressor follows a quasi-steady characteristic

6) Wheel speed is constant

7) Throttle characteristic is parabolic

The compressor characteristic is used to relate the inlet mass flow to the compressor exit pressure:

$$
\mathrm{P}_{0}+\Delta \mathrm{P}_{\mathrm{c}}\left(\dot{\mathrm{m}}_{1}\right)=\mathrm{P}_{1}
$$


Applying the incompressible, 1-dimensional , momentum equation to the fluid in the inlet duct yields:

$$
\left(P_{1}-P_{p}\right) A_{\text {in }}=\frac{d\left(\rho_{0} A_{\text {in }} L_{c} U\right)}{d t}
$$

The volume of the fluid in the inlet duct is given by the effective length of the inlet ducting and and the compressor passages times the effective area. The effective inlet duct length is defined as:

$$
L_{c}=A_{\text {in }} \int_{0}^{1} \frac{d x}{A(x)}
$$

A detailed calculation of the effective duct length for the Holset HD1 is given by Pinsley. The continuity equation applied to the plenum volume yields:

$$
\dot{\mathrm{m}}_{1}-\dot{\mathrm{m}}_{2}=\frac{\mathrm{d}\left(\rho_{\mathrm{p}} \mathrm{V}_{\mathrm{p}}\right)}{\mathrm{dt}}
$$

The pressure drop across the throttle is given by:

$$
\Delta \mathrm{P}_{\mathrm{t}}\left(\dot{\mathrm{m}}_{2}\right)=\mathrm{P}_{\mathrm{p}}-\mathrm{P}_{0}=\frac{1}{2} \rho_{\mathrm{t}} C_{\mathrm{t}}^{2}
$$

and

$$
\begin{gathered}
\dot{\mathrm{m}}_{2}=\rho_{\mathrm{t}} \mathrm{A}_{\mathrm{t}} \mathrm{C}_{\mathrm{t}} \\
\Delta \mathrm{P}_{\mathrm{t}}\left(\dot{\mathrm{m}}_{2}\right)=\frac{1}{2} \frac{\dot{\mathrm{m}}_{2}^{2}}{\rho_{\mathrm{t}} \mathrm{A}_{\mathrm{t}}^{2}} \\
\text { where: } \rho_{\mathrm{t}}=\frac{\rho_{0}+\rho_{\mathrm{p}}}{2}
\end{gathered}
$$




$$
\dot{\mathrm{m}}_{1}-\dot{\mathrm{m}}_{2}=\mathrm{V}_{\mathrm{p}} \frac{\partial \rho_{\mathrm{p}}}{\partial \mathrm{t}}+\rho_{\mathrm{p}} \frac{\partial \mathrm{V}_{\mathrm{p}}}{\partial \mathrm{t}}
$$

assume the air in the plenum behaves isentropically:

$$
\frac{\partial \rho_{\mathrm{p}}}{\partial \mathrm{t}}=\frac{\rho_{\mathrm{p}}}{\gamma \mathrm{P}_{\mathrm{p}}} \frac{\partial \mathrm{P}_{\mathrm{p}}}{\partial \mathrm{t}}=\frac{1}{\mathrm{a}_{\mathrm{p}}^{2}} \frac{\partial \mathrm{P}_{\mathrm{p}}}{\partial \mathrm{t}}
$$

applying small perturbation theory, cancelling the steady relations and nondimensionalizing yields:

$$
\delta \phi_{1}-\delta \phi_{2}=\frac{\bar{V}_{p} U \omega_{h}}{a_{p}^{2} A_{\text {in }}} \frac{\partial \delta \Psi}{\partial \tau}+\frac{\rho_{p} \bar{V}_{p} \omega_{h} \partial \delta v}{\rho_{0} A_{\text {in }} U \partial t}
$$

Using the follwing definitions:

$$
v=\frac{A_{p} q}{\bar{V}_{p}} \quad ; \quad \omega_{h}=a_{p} \sqrt{\frac{A_{h}}{V_{p} L_{c}}} \text { and } M_{t}=\frac{U}{a_{p}}
$$

The non-dimensional form of the continuity equation shown below can be derived:

$$
\delta \phi_{1}-\delta \phi_{2}=\mathrm{B} \frac{\partial \Psi}{\partial \tau}+\frac{\rho_{\mathrm{p}}}{\rho_{0}} \frac{\mathrm{B}}{\mathrm{M}_{\mathrm{t}}^{2}} \frac{\partial \mathrm{v}}{\partial \tau}
$$

Again applying linear perturbations theory to the throttle characteristic and cancelling the steady state relations, yeilds the following algebraic relation between the perturbation exit mass flow and plenum pressure:

$$
\delta \Psi=\frac{\rho_{0}}{\rho_{\mathrm{l}}} \frac{\phi}{\widetilde{\mathrm{A}}_{\mathrm{t}}^{2}} \delta \phi_{2}
$$


The wall dynamics are given as follows:

$$
m \ddot{q}+c \dot{q}+k q=\left(P_{p}-P_{o}\right) A_{p}
$$

where $q$ is defined as the position of the wall away from the equilibrium position of the wall.

Assuming small perturbations about the steady state conditions and nondimensionalizing leads to the the following relations:

Combining the inlet duct momentum equation and the compressor characteristic yields:

$$
\mathrm{P}_{0}+\Delta \mathrm{P}_{\mathrm{c}}\left(\dot{\mathrm{m}}_{1}\right)-\mathrm{P}_{\mathrm{p}}=\frac{\mathrm{L}_{\mathrm{c}}}{\mathrm{A}_{\mathrm{in}}} \frac{\partial \dot{\mathrm{m}}_{1}}{\partial \mathrm{t}}
$$

applying linearized small perturbation theory and cancelling the steady state relations yields:

$$
\frac{\partial \Delta \mathrm{P}_{\mathrm{c}}}{\partial \dot{\mathrm{m}}} \delta \dot{\mathrm{m}}_{1}-\delta \mathrm{P}_{\mathrm{p}}=\frac{\mathrm{L}_{\mathrm{c}}}{\mathrm{A}_{\mathrm{in}}} \frac{\partial\left(\delta \dot{\mathrm{m}}_{1}\right)}{\partial \mathrm{t}}
$$

defining: $\quad \Psi=\frac{\Delta \mathrm{P}_{\mathrm{c}}}{\rho \mathrm{U}^{2}}$ and $\phi=\frac{\dot{\mathrm{m}}}{\rho_{0} \mathrm{~A}_{\mathrm{in}} \mathrm{U}}$

and: $\quad \tau=\omega_{h} t$ and $\frac{\partial}{\partial t}=\omega_{h} \frac{\partial}{\partial \tau}$

The non-dimensional inlet momentum equation is given as:

$$
\frac{\partial \Psi}{\partial \phi} \delta \phi_{1}-\delta \Psi=\frac{1}{\mathrm{~B}} \delta \dot{\phi_{1}}
$$

where $B$ is defined as:

$$
B=\frac{U}{\omega_{h} L_{c}}
$$

Applying the same procedures to the plenum continuity equation: 
Using the definitions of non-dimensional wall motion and time, the equation of motion for the wall can be written:

$$
m \frac{V_{p}}{A_{p}} \omega_{h}^{2} \frac{d^{2}}{d \tau^{2}}(\delta v)+c \frac{V_{p}}{A_{p}} \omega_{h} \frac{d}{d \tau}(\delta v)+k \frac{V_{p}}{A_{p}}(\delta v)=A_{p} \delta P_{p}
$$

define the following non-dimensional control parameters:

$$
\begin{aligned}
& W=\frac{\rho_{0} A_{p}^{2} L_{c}^{2}}{m V_{p}} \\
& \zeta^{\prime}=\frac{c}{2 m \omega_{p}} \\
& Q^{\prime}=\frac{\omega_{p}}{\omega_{h}}
\end{aligned}
$$

and the wall dynamics can be written in non-dimensional form as:

$$
\frac{d^{2} \delta v}{d \tau^{2}}+2 \zeta^{\prime} Q^{\prime} \frac{d \delta v}{d \tau}+Q^{\prime 2} \delta v=W B^{2} \delta \Psi
$$

Using the algebraic relation between the $\delta \Psi$ and $\delta \phi_{2}$, the throttle characteristic can be used to elimiate the $\delta \Psi$ from the set of non-dimensional equations. Also defining:

$$
\eta=\frac{d v}{d \tau}
$$

as the non-dimensional wall velocity to reduce the order of the wall dynamics equation, the governing equation can be expressed as follows:

$$
\begin{gathered}
{\left[\frac{S}{B}-\frac{\partial \Psi}{\partial \phi}\right] \delta \phi_{1}+\frac{\rho_{0}}{\rho_{t}} \frac{\phi}{\widehat{A}_{t}^{2}} \delta \phi_{2}=0} \\
\delta \phi_{1}-\left(1+B s \frac{\rho_{0}}{\rho_{t}} \frac{\phi}{\widehat{A}_{t}^{2}}\right) \delta \phi_{2}-\frac{\rho_{p}}{\rho_{0}} \frac{B}{M_{t}^{2}} \delta \eta=0
\end{gathered}
$$




$$
(s+2 \zeta Q) \delta \eta+Q^{2} \delta v-W B^{2} \frac{\rho_{0}}{\rho_{t}} \frac{\phi}{\widehat{A}_{t}^{2}} \delta \phi_{2}=0
$$

Rearranging these equations and using the steady state relation:

$$
\widehat{A}_{t}^{2}=\frac{\rho_{0}}{\rho_{t}} \frac{\phi^{2}}{2 \Psi}
$$

yields the following stability matrix:

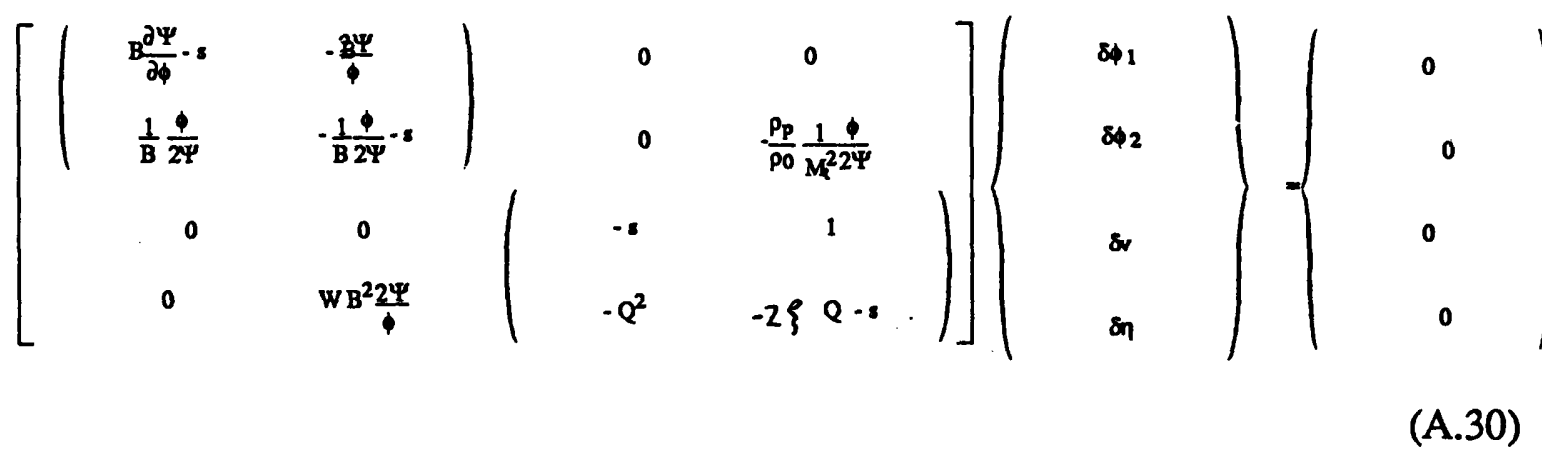

Since the control parameters $\mathrm{Q}^{\prime}$ and $\zeta^{\prime}$ are referenced to the Helmholtz frequency of the compression system which varies slightly with operating conditions, the control parameters were modified so that they remain independent of operatiing conditions.

Substitution of the following corrected control parameters yields the stability matrix given in Section 2.3.

$$
\mathrm{Q}=\mathrm{Q}^{\prime} \sqrt{\frac{\mathrm{P}_{0}}{\rho_{\mathrm{p}}}} \text { and } \zeta=\zeta^{\prime} \sqrt{\frac{\mathrm{P}_{\mathrm{p}}}{\mathrm{P}_{0}} \frac{\mathrm{P}_{0}}{\rho_{\mathrm{p}}}}
$$


Expanding the determinant for the $2 \times 2$ system governing the fixed wall compression system dynamics leads to the characteristic equation shown below:

$$
\begin{aligned}
& s^{2}+\left(\frac{1}{\frac{2 \Psi}{\phi}}-B \frac{\partial \Psi}{\partial \phi}\right) s+\left(1-\frac{\partial \Psi}{\partial \phi} \frac{\phi}{2 \Psi}\right) \\
& \text { Damping term }
\end{aligned}
$$

Dynamic instability occurs when the damping term becomes negative, which occurs when:

(fixed wall stability limit)

$$
\frac{\partial \Psi}{\partial \phi}>\frac{1}{\mathrm{~B}^{2} \frac{2 \Psi}{\phi}}
$$




\section{Appendix B}

\section{Alternative Schemes for Passive Surge Supression}

In addition to the flexible plenum wall control strategy, three other methods to passively stabilize surge using structural or aerodynamic feedback were investigated. The unifying theme in each of these methods is that the system dynamics are modified to increase unsteady energy dissipation thus extending the stable operating range.

\section{The Flexible Throttle Method}

A schematic of a compression system with a flexible throttle is shown below.

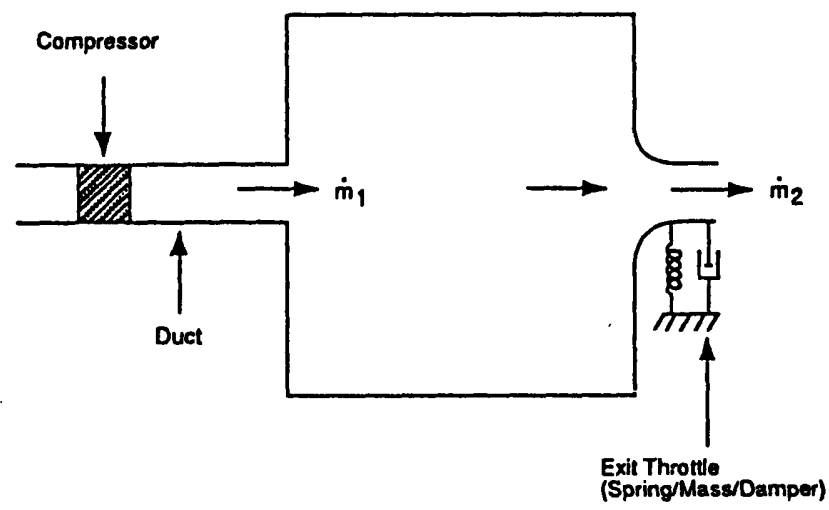

\section{Passive Throttle Control}

The flexible throttle method was developed by Chen ${ }^{10}$. The throttling valve is modelled as a mass-spring-damper system and the throttle area responds to perturbations in plenum pressure. A detailed schematic of the flexible throttle valve is shown below.

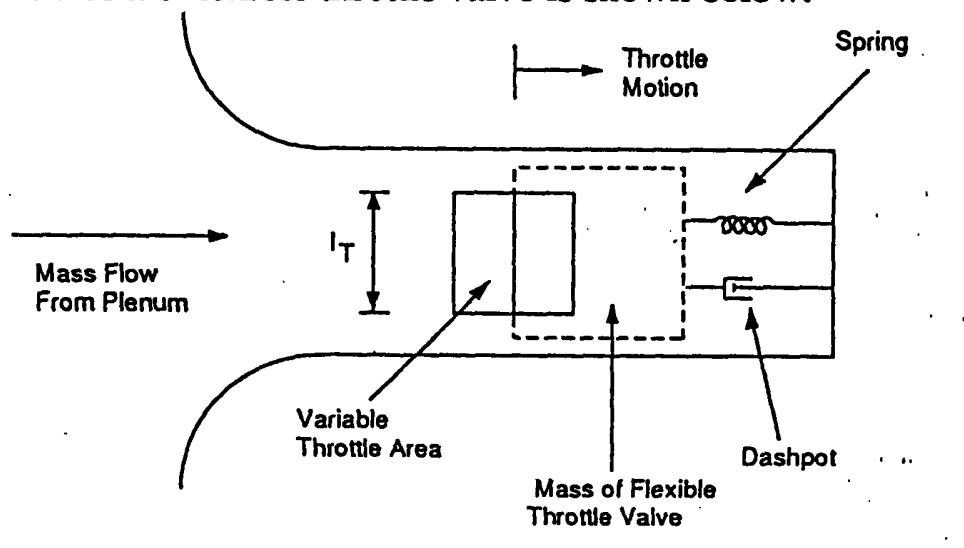


By using a lumped parameter model and applying an analysis similar to that developed for the flexible plenum wall, the following stability matrix can be derived.

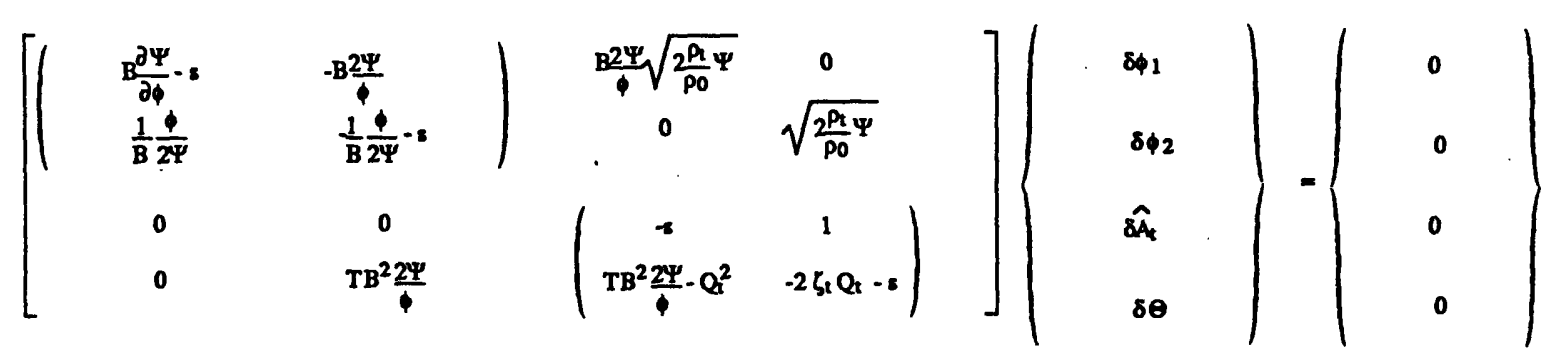

where B, $\phi$ and $\Psi$ are the stability parameter, mass flow coefficient, and the pressure rise coefficient described in the text. The throttle control parameters are defined as follows:

$$
\zeta_{\mathrm{t}}=\frac{c_{\mathrm{t}}}{2 \mathrm{~m}_{\mathrm{t}} \omega_{\mathrm{t}}} \quad \mathrm{Q}_{\mathrm{t}}=\frac{\omega_{\mathrm{t}}}{\omega_{\mathrm{h}}} \quad \mathrm{T}=\frac{2 \rho_{0} \mathrm{~L}_{\mathrm{t}} \hat{\mathrm{A}}_{\mathrm{v}} \mathrm{L}_{\mathrm{c}}^{2}}{\mathrm{~m}_{\mathrm{t}}}
$$

where $A_{V}$ is the area of throttle valve mass-spring-damper over which the plenum pressure acts. The perturbation quantities are defined as follows:

$$
\begin{aligned}
& \delta \phi_{1}=\text { inlet mass flow } \\
& \delta \phi_{2}=\text { exit mass flow } \\
& \delta A_{t}=\text { non-dimensional throttle area } \\
& \delta \theta=\text { non-dimensional rate of change of throttle area }
\end{aligned}
$$

A root locus plot for a properly tuned flexible throttle system is shown below.

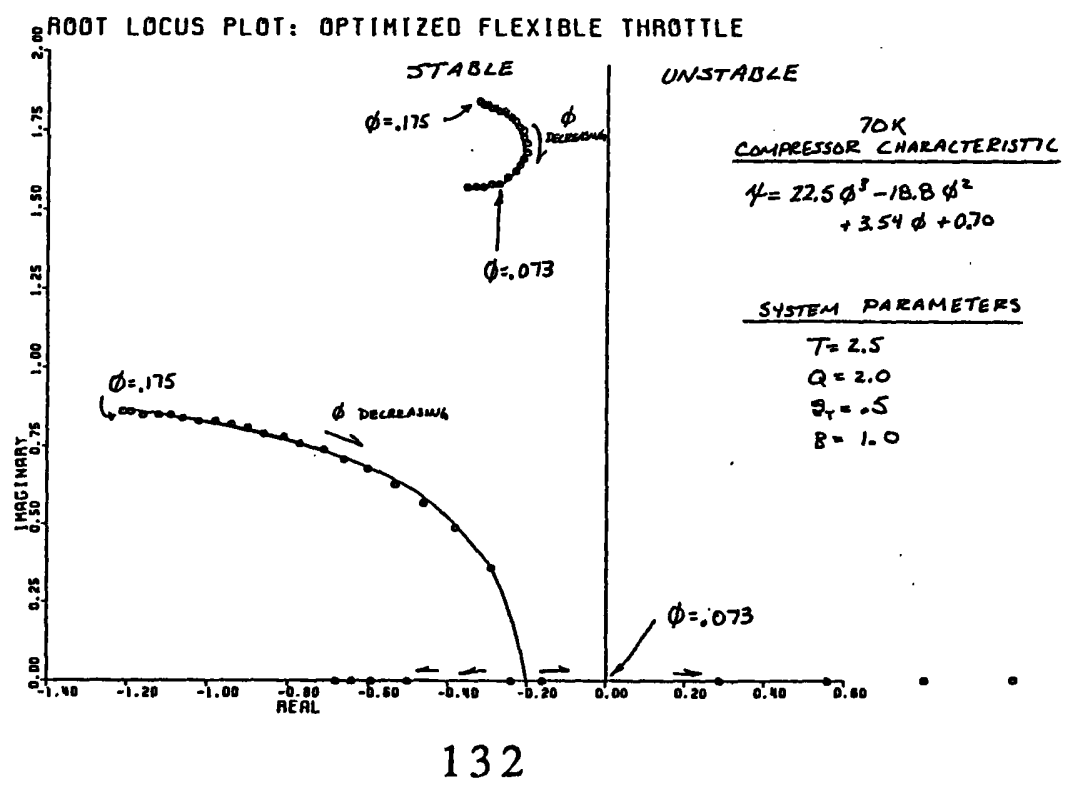


The maximum controllable slope ( $\partial \Psi / \partial \phi$ ) for the flexible throttle system at $B=1.0$ is shown to be approximately $30 \%$ higher than the flexible plenum wall system developed in the text.

The main stabilizing mechanism in the flexible plenum wall method is the modification of the unsteady throttle slope on a pressure drop versus mass flow map. The flexible valve reduces the apparent throttle slope, and thereby, allows the flexible throttle to damp out flow disturbances more effectively than a rigid throttle. This mechanism is different in nature than the one in the flexible plenum wall method where the main stabilizing mechanism is the energy dissipation in the wall motion itself.

\section{The Auxiliary Throttle Method}

A schematic of the auxiliary throttle method is shown below.

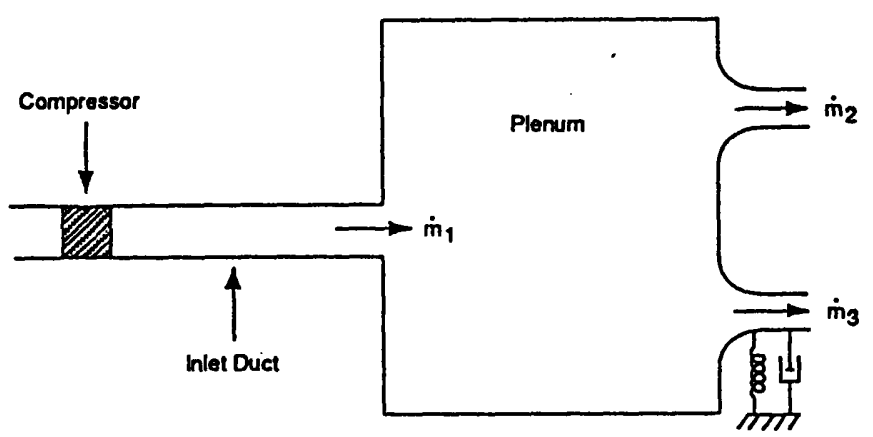

\section{Auxiliary Throttle}

The auxiliary throttle method is similar to the flexible throttle method; however, in this method, the amount of steady state mass flow through the flexible throttle is variable.

The stability matrix for this method is shown below.

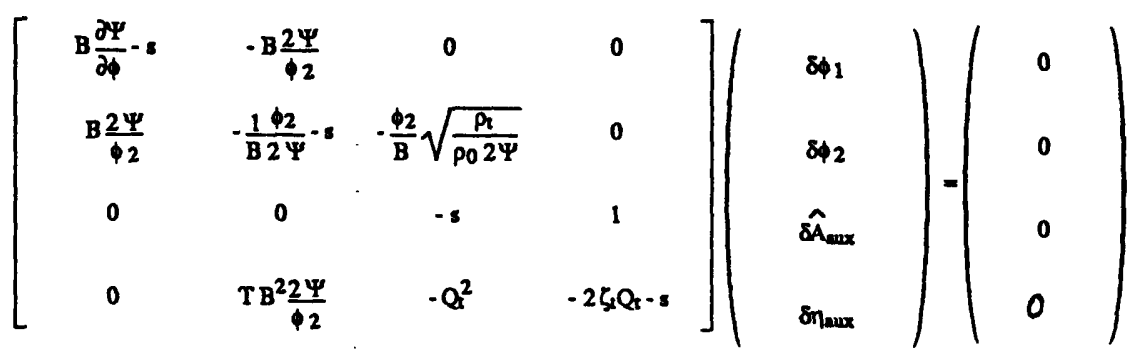


The system operating and control parameters are the same as the flexible throttle. The root locus plot of a tuned auxiliary throttle system is identical to the flexible throttle root locus. The linear stability of the auxiliary throttle is independent of the ratio of steady state mass flow going through the rigid and flexible throttles.

\section{The Aerodynamic Damper}

The aerodynamic damper method employs an additional, damped Helmholtz resonator to increase the unsteady energy dissipation and, thereby, extend the stable operating range. A schematic of the aerodynamic damper is shown below.

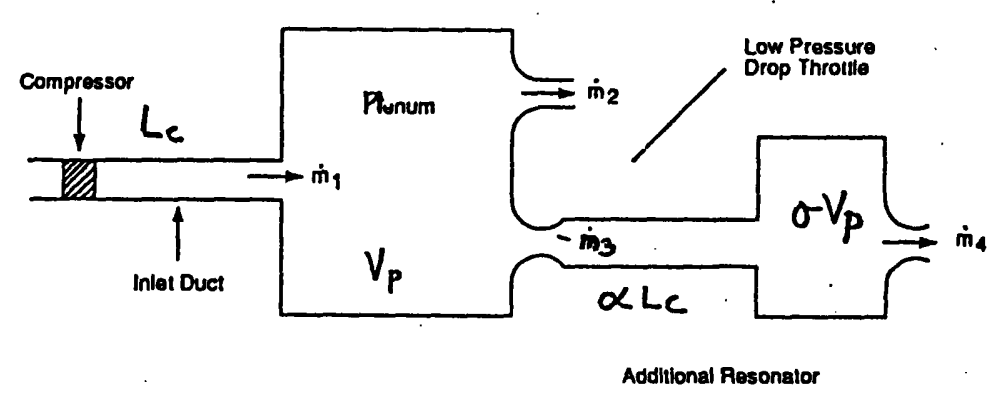

\section{Aerodynamic Damper}

The stability matrix for the aerodynamic damper is shown below.

$$
\left[\begin{array}{cccc}
B \frac{\partial \Psi}{\partial \phi}-8 & -B \frac{2 \Psi}{\phi 2} & 0 & 0 \\
B \frac{2 \Psi}{\phi 2} & -\frac{1}{B} \frac{\phi 2}{2 \Psi}-8 & B \frac{2 \Psi}{\phi 2} & 0 \\
0 & \frac{B 2 \Psi}{\alpha \phi 2} & -\frac{B 2 \Psi_{t}-8}{\alpha \phi_{3}} & \frac{B 2\left(\Psi-\Psi_{t}\right)}{\alpha}-s \\
0 & 0 & \frac{1}{\sigma B 2\left(\Psi-\Psi_{t}\right)} & -\frac{1}{\sigma B 2\left(\Psi-\Psi_{t}\right)}-8
\end{array}\right]\left(\begin{array}{c}
\delta \phi 1 \\
\delta \phi 2 \\
\delta \phi 3 \\
\delta \phi 4
\end{array}\right)=\left(\begin{array}{c}
0 \\
0 \\
0 \\
0
\end{array}\right)
$$

where

$$
\begin{aligned}
& \alpha=\text { the ratio of effective inlet duct lengths of the to resonators } \\
& \sigma=\text { the ratio of volumes between the two systems } \\
& \Psi_{t}=\text { the pressure drop across the low pressure drop throttle }
\end{aligned}
$$


The perturbation quantities represent perturbations of the mass flow coefficients shown in the schematic.

A root locus plot for a tuned aerodynamic damper is shown below.

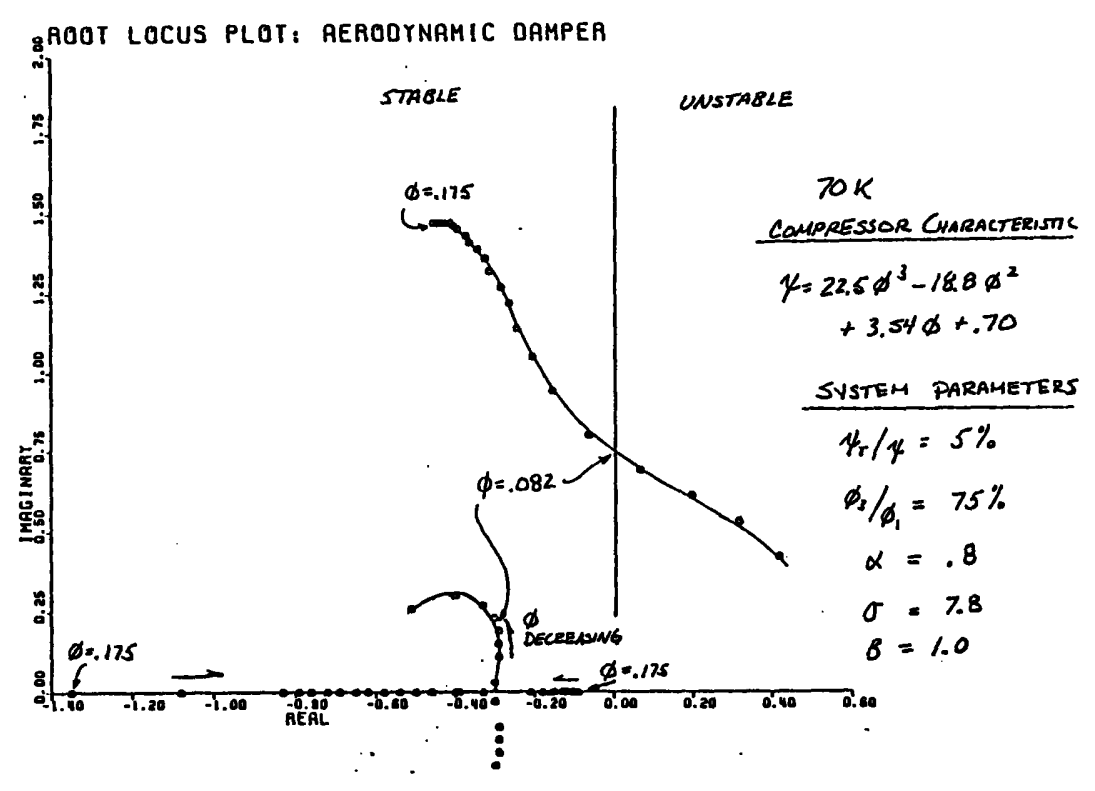

The control effectiveness of the aerodynamic damper system is shown to be roughly equivalent to the flexible plenum wall control method, however, in this method, there is a steady state performance penalty due to losses through the low pressure drop throttle. 